\title{
Antidepressant drugs act by directly binding to TRKB neurotrophin receptors
}

Casarotto, Plinio C.

2021-03-04

Casarotto , P C , Girych , M , Fred , S M , Kovaleva , V , Moliner , R , Enkavi , G, Biojone , C , Cannarozzo , C , Sahu , M P , Kaurinkoski , K, Brunello, C A , Steinzeig , A , Winkel , F , Patil , S , Vestring , S , Serchov , T, Diniz , C R A F , Laukkanen , L , Cardon , I , Antila , H , Rog , T , Piepponen , T P , Bramham , C R, Normann , C , Lauri , S E , Saarma , M , Vattulainen , I \& Castren, E 2021, ' Antidepressant drugs act by directly binding to TRKB neurotrophin receptors ' , Cell , vol. 184 , no. 5 , pp. 1299-+ . https://doi.org/10.1016/j.cell.2021.01.034

http://hdl.handle.net/10138/329304

https://doi.org/10.1016/j.cell.2021.01.034

cc_by

publishedVersion

Downloaded from Helda, University of Helsinki institutional repository.

This is an electronic reprint of the original article.

This reprint may differ from the original in pagination and typographic detail.

Please cite the original version. 


\section{Antidepressant drugs act by directly binding to TRKB neurotrophin receptors}

\section{Graphical Abstract}

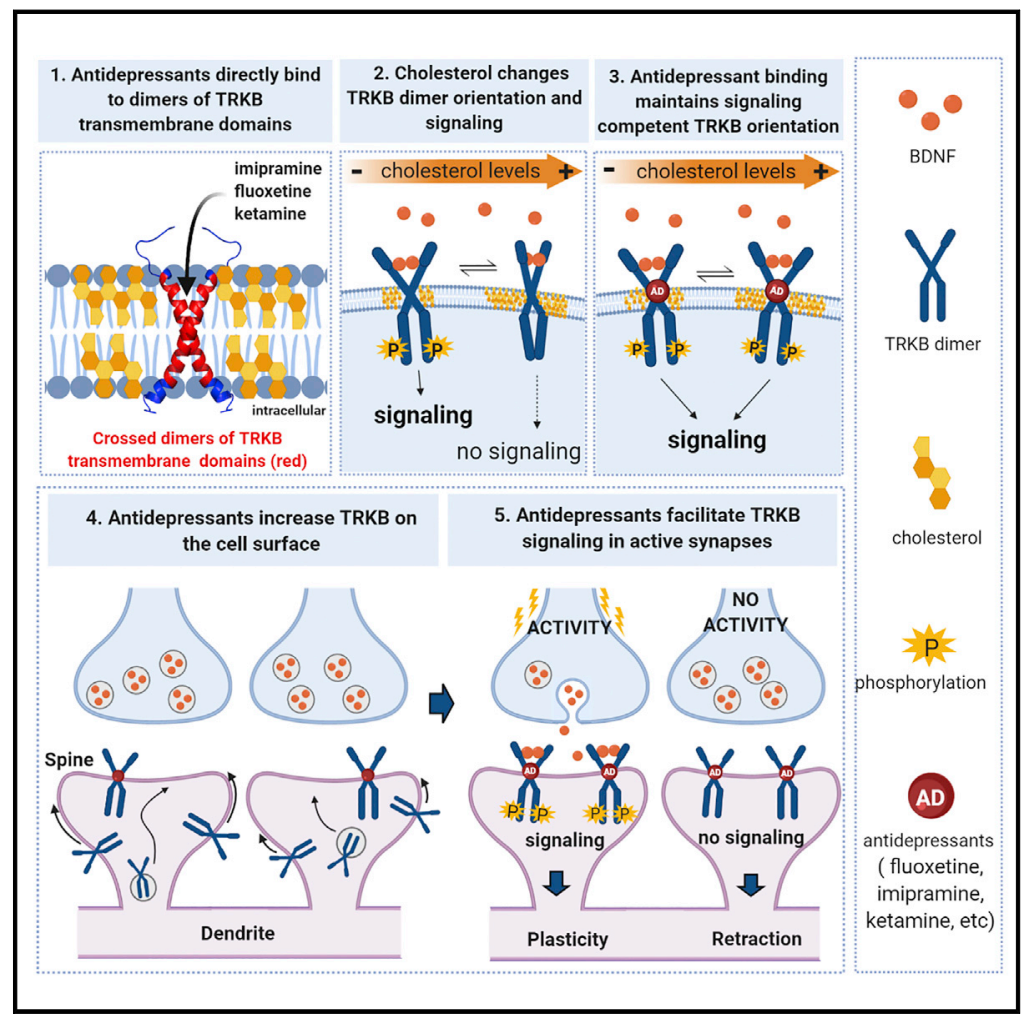

\section{Highlights}

- Several antidepressants, including SSRls and ketamine, directly bind to TRKB

- TRKB dimerization at transmembrane region forms a binding pocket for fluoxetine

- Antidepressant binding to TRKB facilitates BDNF action and plasticity

- Point mutation in TRKB transmembrane region blocks the effects of antidepressants

\section{Authors}

Plinio C. Casarotto, Mykhailo Girych, Senem M. Fred, ..., Mart Saarma, Ilpo Vattulainen, Eero Castrén

\section{Correspondence}

eero.castren@helsinki.fi

\section{In Brief}

Direct binding of both typical and fastacting antidepressants to the BDNF receptor TRKB accounts for cell biological and behavioral actions of antidepressants. This mechanism directly connects antidepressant action to neuronal plasticity and may explain the slow action of typical antidepressants. 


\title{
Antidepressant drugs act by directly binding to TRKB neurotrophin receptors
}

\author{
Plinio C. Casarotto, ${ }^{1}$ Mykhailo Girych, ${ }^{2}$ Senem M. Fred, ${ }^{1}$ Vera Kovaleva, ${ }^{3}$ Rafael Moliner, ${ }^{1}$ Giray Enkavi, ${ }^{2}$ \\ Caroline Biojone, ${ }^{1}$ Cecilia Cannarozzo, ${ }^{1}$ Madhusmita Pryiadrashini Sahu, ${ }^{1}$ Katja Kaurinkoski, ${ }^{1}$ Cecilia A. Brunello, ${ }^{1}$ \\ Anna Steinzeig, ${ }^{1}$ Frederike Winkel, ${ }^{1}$ Sudarshan Patil, ${ }^{4}$ Stefan Vestring, ${ }^{5,6}$ Tsvetan Serchov, ${ }^{5,16}$ Cassiano R.A.F. Diniz, ${ }^{1,7}$ \\ Liina Laukkanen, ${ }^{1}$ Iseline Cardon, ${ }^{1,8,9}$ Hanna Antila, ${ }^{1,10}$ Tomasz Rog, ${ }^{2}$ Timo Petteri Piepponen, ${ }^{11}$ Clive R. Bramham, ${ }^{4}$ \\ Claus Normann, ${ }^{5,12}$ Sari E. Lauri, ${ }^{1,13}$ Mart Saarma, ${ }^{3}$ Ilpo Vattulainen, ${ }^{2,14}$ and Eero Castrén ${ }^{1,15,17, *}$ \\ ${ }^{1}$ Neuroscience Center-HILIFE, University of Helsinki, Helsinki, Finland \\ 2Department of Physics, University of Helsinki, Helsinki, Finland \\ ${ }^{3}$ Institute of Biotechnology-HILIFE, University of Helsinki, Helsinki, Finland \\ ${ }^{4}$ Department of Biomedicine and KG Jebsen Center for Research on Neuropsychiatric Disorders, University of Bergen, Bergen, Norway \\ ${ }^{5}$ Department of Psychiatry and Psychotherapy, Medical Center-University of Freiburg, Faculty of Medicine, University of Freiburg, Freiburg, \\ Germany \\ ${ }^{6}$ Berta-Ottenstein-Programme for Clinician Scientists, Faculty of Medicine, University of Freiburg, Freiburg, Germany \\ ${ }^{7}$ Department of Pharmacology, Ribeirão Preto Medical School, University of São Paulo, São Paul, Brazil \\ ${ }^{8}$ Brain Master Program, Faculty of Science, Aix-Marseille Université, Marseille, France \\ ${ }^{9}$ Department of Psychiatry, University of Regensburg, Regenburg, Germany \\ ${ }^{10}$ Department of Neuroscience, Perelman School of Medicine, University of Pennsylvania, Philadelphia, PA, USA \\ ${ }^{11}$ Division of Pharmacology and Pharmacotherapy, Faculty of Pharmacy, University of Helsinki, Helsinki, Finland \\ ${ }^{12}$ Center for Basics in Neuromodulation (NeuroModul Basics), University of Freiburg, Freiburg, Germany \\ ${ }_{13}$ Molecular and Integrative Biosciences Research Program, University of Helsinki, Helsinki, Finland \\ ${ }^{14}$ Computational Physics Laboratory, Tampere University, Tampere, Finland \\ ${ }^{15}$ E.C. dedicates this paper to the memory of Dr. Ronald S. Duman \\ ${ }^{16}$ Present address: Centre National de la Recherche Scientifique (CNRS), Université de Strasbourg, Institut des Neurosciences Cellulaires et \\ Intégratives, 67000 Strasbourg, France \\ 17Lead contact \\ ${ }^{*}$ Correspondence: eero.castren@helsinki.fi \\ https://doi.org/10.1016/j.cell.2021.01.034
}

\section{SUMMARY}

It is unclear how binding of antidepressant drugs to their targets gives rise to the clinical antidepressant effect. We discovered that the transmembrane domain of tyrosine kinase receptor 2 (TRKB), the brain-derived neurotrophic factor (BDNF) receptor that promotes neuronal plasticity and antidepressant responses, has a cholesterol-sensing function that mediates synaptic effects of cholesterol. We then found that both typical and fast-acting antidepressants directly bind to TRKB, thereby facilitating synaptic localization of TRKB and its activation by BDNF. Extensive computational approaches including atomistic molecular dynamics simulations revealed a binding site at the transmembrane region of TRKB dimers. Mutation of the TRKB antidepressant-binding motif impaired cellular, behavioral, and plasticity-promoting responses to antidepressants in vitro and in vivo. We suggest that binding to TRKB and allosteric facilitation of BDNF signaling is the common mechanism for antidepressant action, which may explain why typical antidepressants act slowly and how molecular effects of antidepressants are translated into clinical mood recovery.

\section{INTRODUCTION}

Several targets for antidepressant $(A D)$ drug action have been identified, but it is not clear how binding to these targets translates into clinical effects. Typical ADs such as tricyclic ADs (TCA), serotonin selective reuptake inhibitors (SSRI), and monoamine oxidase inhibitors (MAOI), increase the synaptic levels of monoamines by inhibiting their reuptake or metabolism, but it is unclear why their clinical effects are delayed, while the effects on monoamines are fast (Belmaker and Agam, 2008; Malhi and Mann, 2018). The rapid AD effect of ketamine (KET) is attributed to inhibition of NMDAtype glutamate receptors (Abdallah et al., 2015; Berman et al., 2000; Zarate et al., 2006). However, 2R,6R-hydroxynorketamine (R,R-HNK), a KET metabolite with AD-like activity, exhibits low affinity to NMDA receptors, which has called the role of NMDA receptors in the KET action into question (Zanos et al., 2016, 2018).

Essentially all ADs, including KET and R,R-HNK, increase the expression and signaling of brain-derived neurotrophic factor 
(BDNF) through neurotrophic tyrosine kinase receptor 2 (TRKB) (Autry and Monteggia, 2012; Castrén and Antila, 2017; Duman and Monteggia, 2006). The effects of SSRIs and KET on BDNF signaling have been considered to be indirect, through the inhibition of serotonin transporter $(5 \mathrm{HTT})$ and NMDA receptors, respectively. BDNF mimics the effects of ADs in rodents and inhibiting TRKB signaling prevents their behavioral effects (Duman and Monteggia, 2006; Saarelainen et al., 2003). Activation of TRKB is a critical mediator of activity-dependent synaptic plasticity (Park and Poo, 2013), and the AD-induced TRKB signaling reactivates a state of juvenile-like plasticity in the adult brain, which has been suggested to underlie the effects of ADs on mood (Castrén and Antila, 2017; Karpova et al., 2011; Maya Vetencourt et al., 2008).

TRKB signaling is bidirectionally linked to brain cholesterol (CHOL) metabolism. BDNF promotes production of $\mathrm{CHOL}$ in neurons (Suzuki et al., 2007; Zonta and Minichiello, 2013) and CHOL regulates TRKB signaling (Pereira and Chao, 2007; Suzuki et al., 2004). CHOL is essential for neuronal maturation and proper synaptic transmission (Martin et al., 2014; Mauch et al., 2001), but it does not pass the blood-brain barrier, therefore, neurons are dependent on $\mathrm{CHOL}$ synthesized by astrocytes and transported through an ApoE-mediated mechanism (Pfrieger and Ungerer, 2011). Synaptic CHOL levels are low during the embryonic and early postnatal life but strongly increase during the $3^{\text {rd }}$ postnatal week in mice (Suzuki et al., 2004; Tulodziecka et al., 2016), which coincides with the increase in BDNF expression and appearance of ADs effects on TRKB (Di Lieto et al., 2012). Many ADs interact with phospholipids and accumulate in CHOL-rich membrane domains, such as lipid rafts (Erb et al., 2016; Wray et al., 2019).

These data prompted us to investigate the potential interactions between TRKB, CHOL, and ADs. We found that the TRKB transmembrane domain (TMD) senses changes in the cell membrane $\mathrm{CHOL}$ levels, and we elucidated its mechanism. Furthermore, we found that different $A D$ drugs directly bind to a site formed by a dimer of TRKB TMDs, thereby facilitating cell surface expression of TRKB and promoting BDNF signaling. These data suggest that direct binding to TRKB and promotion of BDNF-mediated plasticity is a mechanism of action for $A D$ drugs.

\section{RESULTS}

\section{Cholesterol sensing by TRKB}

$\mathrm{CHOL}$ is known to promote neuronal maturation and plasticity, but how it exerts these effects is unclear (Mauch et al., 2001; Pfrieger and Ungerer, 2011). CHOL is proposed to interact with proteins through the so-called $\mathrm{CHOL}$-recognition and alignment consensus (CRAC) domain or its inverted version CARC (Fantini et al., 2019). We identified a CARC motif in the TRKB transmembrane (TM) region. This sequence is specific to TRKB and is not present in other TRK receptors (Figure 1A), suggesting that $\mathrm{CHOL}$ might directly interact with TRKB. Indeed, addition of $\mathrm{CHOL}$ at $20 \mu \mathrm{M}$ to the culture media enhanced TRKB phosphorylation (pTRKB) by BDNF $(10 \mathrm{ng} / \mathrm{mL})$ in primary cortical neurons (Figure 1B). However, at higher concentrations $(50-100 \mu \mathrm{M})$, $\mathrm{CHOL}$ suppressed the effects of BDNF (Figure 1B). CHOL pro- moted the interaction of TRKB, but not of TRKA, with phospholipase C- $\gamma 1$ (PLC- $\gamma 1$ ) (Figures S1A-S1E), a critical mediator of TRKB intracellular signaling (Minichiello et al., 2002), and this effect was blocked by beta-cyclodextrin ( $\beta C D X)$, a CHOL-sequestering agent, at a dose that counteracts $\mathrm{CHOL}$ effects (Figures $1 \mathrm{C}$ and S1F). Microscale thermophoresis (MST) (Jerabek-Willemsen et al., 2014) experiments demonstrated that $\mathrm{CHOL}(10-100 \mu \mathrm{M})$ directly interacts with GFP-TRKB in HEK293T cell lysates with an affinity of $\sim 20 \mu \mathrm{M}$ (Figure 1E).

TRKB mostly resides in intracellular vesicles not accessible to BDNF (Du et al., 2000; Haapasalo et al., 2002; Meyer-Franke et al., 1998). We found that $\mathrm{CHOL}$ treatment increased cell surface translocation of TRKB (Figure S1C). The effects of BDNF on TRKB-PLC- $\gamma 1$ interaction (Figure 1D) and on the neurite branching in cultured neurons (Figures S1G-S1K) were prevented by a CHOL synthesis inhibitor pravastatin $(1 \mu \mathrm{M} /$ 3 days), as reported previously (Suzuki et al., 2004). At $2 \mu \mathrm{M}$ for 5 days, pravastatin reduced neuronal survival that was rescued by $\mathrm{CHOL}(20 \mu \mathrm{M})$, but not by BDNF (Figures S1L and $\mathrm{S} 1 \mathrm{M})$.

Mutation of TRKB tyrosine 433, a predicted key residue in the CARC motif (Fantini and Barrantes, 2013) to phenylalanine (TRKB.Y433F), did not influence the binding affinity of BDNF $($ TRKB.wt $=3.1 \mathrm{pM}$; TRKB.Y433F $=2.9 \mathrm{pM})$ (Figure S2A), but it compromised $\mathrm{CHOL}$ sensing of TRKB (Figure 1E) and reduced the BDNF-induced increase in the phosphorylation of TRKB at the PLC- $\gamma 1$ interaction site Y816, but not at Y515 (Figures S2B and S2C). Split luciferase protein complementation assay (Merezhko et al., 2020) indicated that although Y433F mutation did not influence the basal TRKB dimerization, it compromised BDNF-induced increase in TRKB dimerization (Figure S2D). Furthermore, BDNF-induced translocation of TRKB.Y433F to lipid rafts (Figure S2F) and its interaction with the raft-restricted FYN (Pereira and Chao, 2007) was reduced when compared to the wild-type TRKB (Figure S2E). These data indicate that the Y433 in the TRKB CARC domain is important for BDNF-induced translocation of TRKB to lipid-raft regions on the neuronal surface, thereby promoting BDNF signaling.

\section{Modeling of cholesterol-TRKB interaction}

We next used atomistic molecular dynamics (MD) simulations to investigate the organization of TRKB TMD dimers (Table S1). Using a docking algorithm, we modeled five TMD dimer structures to initiate MD simulations, which showed that only one of them is stable in a phosphatidylcholine bilayer with $20-40 \mathrm{~mol} \% \mathrm{CHOL}$. The stable structure features a cross-like conformation, where the two TMD helices interact at ${ }^{439} \mathrm{AXXXG}^{443}$ (Figures 1G-11), a GXXXG-like dimerization motif (Figure 1G) (Li et al., 2012; Senes et al., 2004). A similar cross-like conformation was proposed for the EGF receptor, where the distance between the C-termini of TMDs determines EGF signaling (Arkhipov et al., 2013; Endres et al., 2013; Sinclair et al., 2018). The average distance between the $\mathrm{C}$ termini of TRKB TMDs at CHOL concentrations of 0,20 , and $40 \mathrm{~mol} \%$ was $19.4 \AA, 14.3 \AA$, and $12.4 \AA$, respectively (Figure S3A).

Additional simulations revealed that the conformation of TRKB TMD dimers is sensitive to $\mathrm{CHOL}$. As the fixed-length hydrophobic TMD helices reduce their tilt to match the thicker membrane 
A

\begin{tabular}{|c|c|c|}
\hline UniProt ID & aa res & transmembrane region sequence \\
\hline Q3UFB7 (TRKA) & $416-446$ & ETPFGVSVAVGLAVSAALFLSALLLVNKCG \\
\hline P15209 (TRKB) & $425-458$ & SNREHLSVYAVVVIASVVGFCLLVMLLLLKLARH \\
\hline Q6VNS1 (TRKC) & $425-458$ & PEEDTFGVSIAVGLAAFACVLLVVLFIMINKYGR \\
\hline
\end{tabular}

B

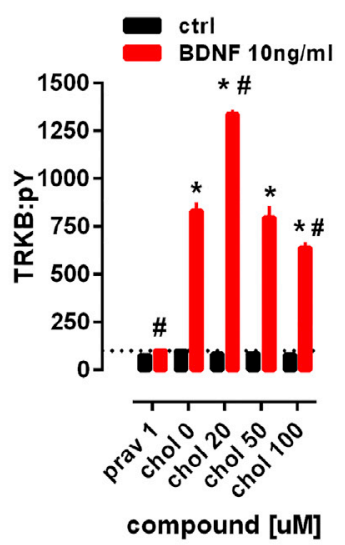

C

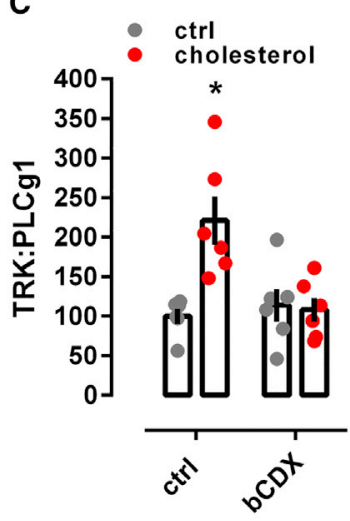

D

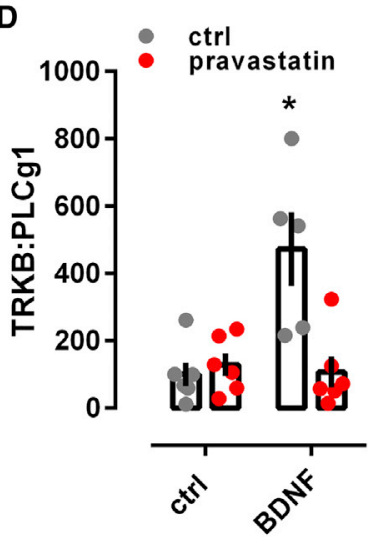

E

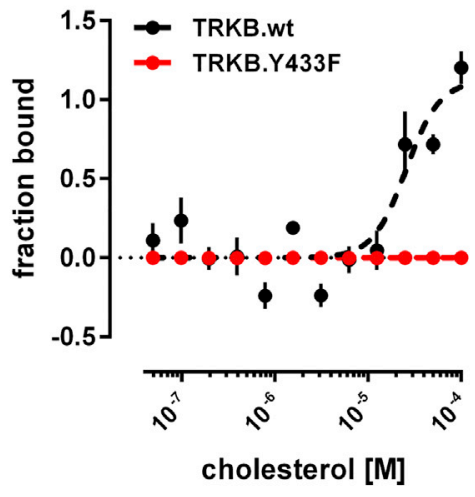

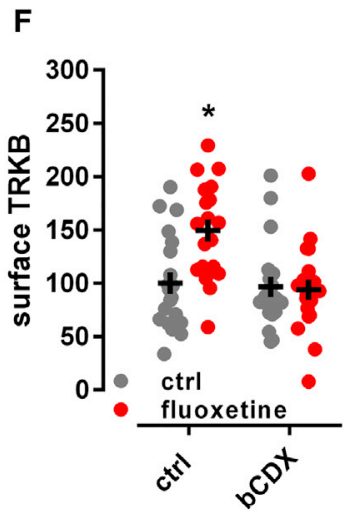

G

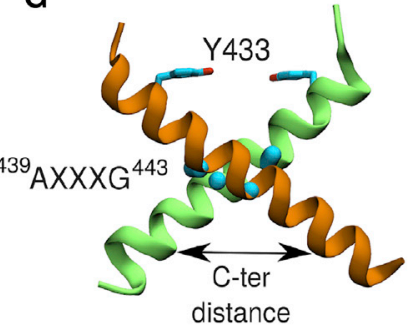

TRKB.wt 0\%chol

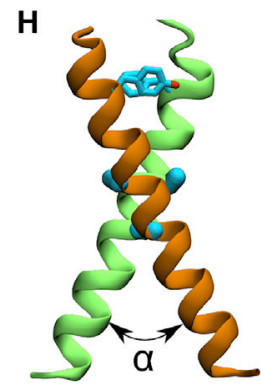

TRKB.wt 20\%chol

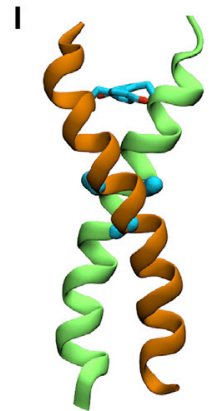

TRKB.wt $40 \%$ chol

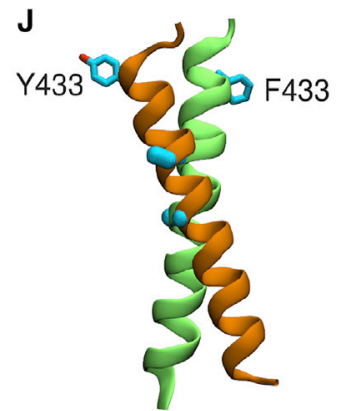

TRKB.Y433F 20\%chol

Figure 1. Cholesterol sensing by TRKB

(A) Identification of CARC motif (red) in the TM domain of TRKB, but not TRKA or TRKC.

(B) Cholesterol promotes the effects of BDNF on TRKB autophosphorylation (TRKB:pY) at moderate, but inhibits BDNF at low or high concentrations (interaction: $F[5,84]=5.654, p=0.0002 ; n=6 / g r o u p)$. Cultured cortical cells received cholesterol ( $15 \mathrm{~min}$ ) followed by BDNF or cholesterol (15 min) and were submitted to ELISA for TRKB:pY.

(C) $\beta$-cyclodextrin (bCDX, 2 mM, $30 \mathrm{~min}$ ) prevents BDNF-induced increase in TRKB-PLC- $\gamma 1$ interaction (TRK:PLCg1) (interaction: $F[1,20]=9.608, p=0.0056$, $\mathrm{n}=6$ /group).

(D) Pravastatin ( $1 \mu \mathrm{M}, 3$ days) also blocks the BDNF-induced increase in TRKB:PLC- $\gamma 1$ interaction (interaction: $F[1,19]=11.23, p=0.003 ; n=5-6)$. ${ }^{*} p<0.05$ from the $\mathrm{ctrl} / \mathrm{ctrl}$ group, $\# p<0.05$ from ctrl/chol0 group, data expressed as mean \pm SEM of percentage from control group.

(E) Microscale thermophoresis demonstrated direct interaction between GFP-tagged TRKB and cholesterol (15 min) in lysates from GFP-TRKB expressing HEK293T cells; mutation of Y433F blocks this interaction in MST (interaction: $F[11,72]=15.25, p<0.0001, n=4$ ).

(F) Fluoxetine-induced increase in TRKB surface exposure is blocked by bCDX (interaction: $F[1,73]=7.022, p=0.0099, n=19-20$ ).

(G-J) Structure of wild-type TRKB (G) in the absence of cholesterol and (H) at cholesterol concentrations of $20 \mathrm{~mol} \%$ and $(\mathrm{I}) 40 \mathrm{~mol} \%$, and $(\mathrm{J})$ for the heterodimer of TRKB.wt and TRKB.Y433F at $20 \mathrm{~mol} \%$. Related to systems 5-8 in Table S1 and Figure S2 for distance and $\alpha$ values between C termini.

See also Figures S1, S2, and S3.

at higher $\mathrm{CHOL}$ concentration, the stable cross-like dimer conformation seen at $20 \mathrm{~mol} \% \mathrm{CHOL}$ switched to a more parallel conformation at $40 \mathrm{~mol} \%$ (Figures $1 \mathrm{H}$ and $1 \mathrm{I})$. The Y433F mutation induced a $40^{\circ}$ rotation of the TMD helices relative to each other (Figure 1J). This compromises the contact at the ${ }^{439} \mathrm{AXXXG}{ }^{443}$ motif and reduces the C-terminal distance of the TMDs (Figures S3A and S3B). In TRKA, which has no CARC or GXXXG-like domains, different $\mathrm{CHOL}$ concentrations did not 


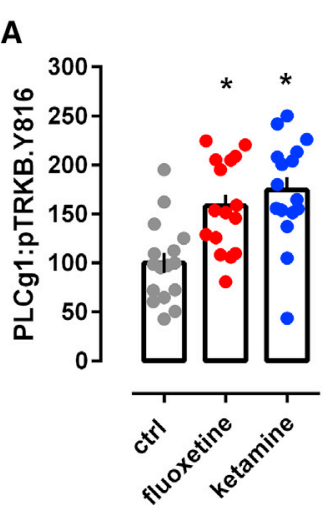

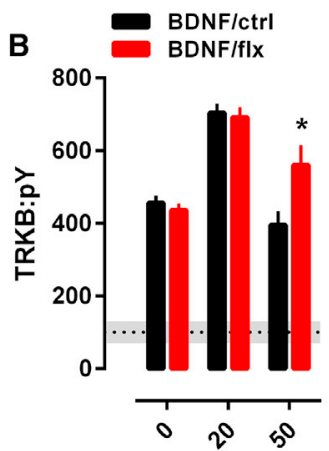

cholesterol [uM]
C

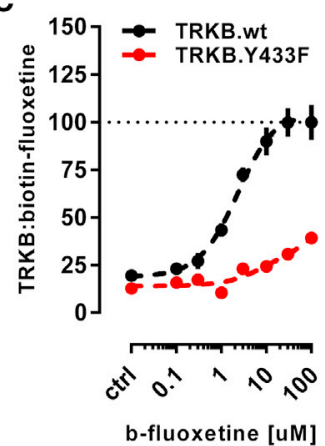

D

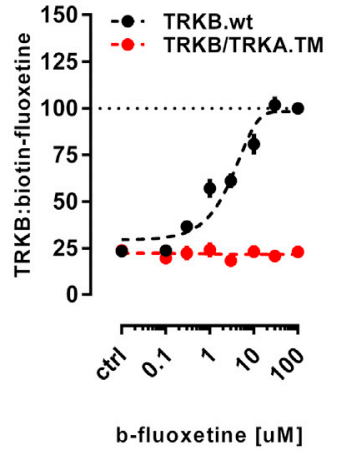

$\mathbf{E}$

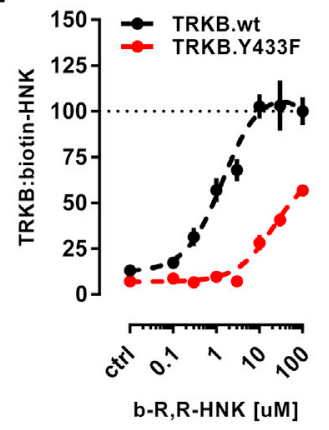

$\mathbf{F}$

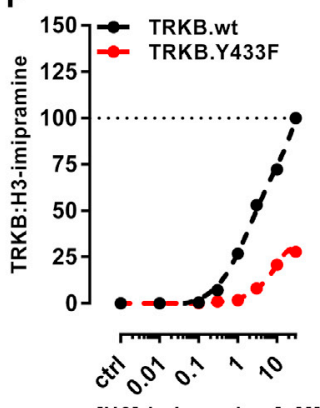

[H3]-imipramine [uM]
G

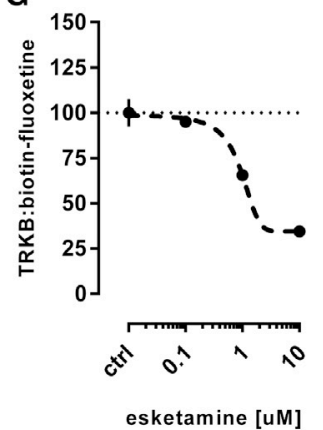

H

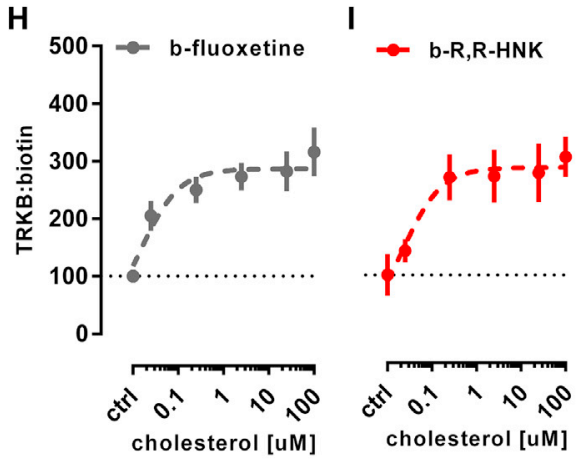

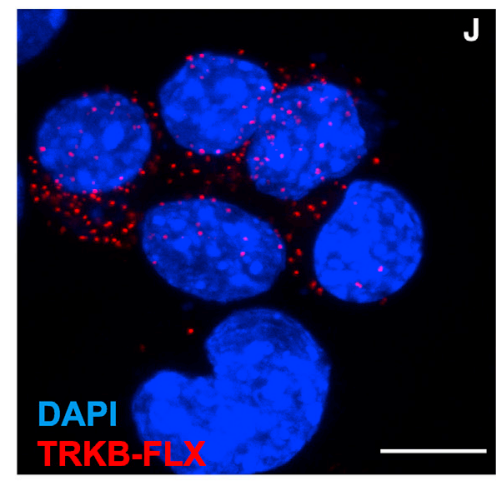

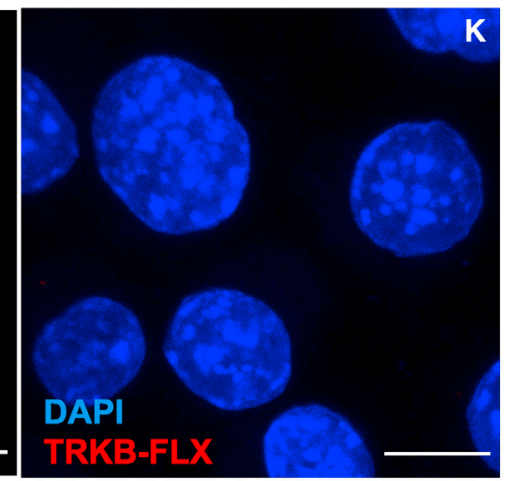

L

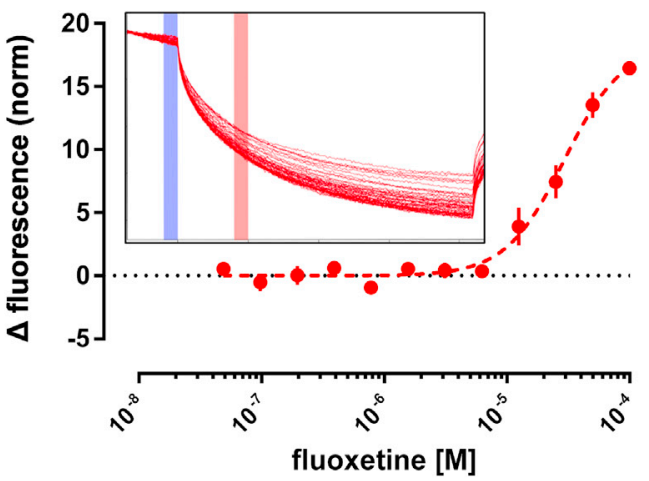

Figure 2. Antidepressants bind to TRKB transmembrane domain

(A) Fluoxetine $(10 \mu \mathrm{M} / 15 \mathrm{~min})$ and ketamine $(10 \mu \mathrm{M} / 15 \mathrm{~min})$ increased pTRKB.Y816 in cortical neurons immunoprecipitated with anti-PLC- $\gamma 1$ (F[2,45] $=11.03$, $p=0.0001, n=16 /$ group).

(B) Fluoxetine facilitates BDNF-induced activation of TRKB under high cholesterol concentrations (interaction: $F[2,132]=5.15, p=0.0070, n=12 / g r o u p$ ) in cultured cortical cells.

( $C$ and D) Biotinylated fluoxetine binds to TRKB in lysates of TRKB expressing HEK cells (interaction: $F[7,153]=16.18, p<0.0001 ; n=6-14$ ), but not (C) to TRKB.Y433F mutant or (D) to TRKB carrying the TMD of TRKA (TRKB/TRKA.TM) (interaction: $F[7,80]=43.75, p<0.0001, n=6 /$ group).

( $E$ and $F$ ) Binding of biotinylated R,R-HNK (interaction: $F[7,160]=14.91, p<0.0001 ; n=6-14)(E)$ and tritiated imipramine (interaction: $F[7,16]=106.1, p<0.0001$; $n=2)(F)$ to TRKB, but not to TRKB.Y433F. Data expressed mean \pm SEM of percentage of binding at $100 \mu M$ for fluoxetine and $R, R-H N K$ or at $30 \mu M$ for imipramine.

(G) Esketamine displaces the interaction of biotinylated fluoxetine $(1 \mu \mathrm{M})$ with TRKB $(n=8 /$ group$)$.

( $H$ and $I)$ Cholesterol facilitates the interaction of $(H)$ biotinylated fluoxetine $(F[5,30]=7.198, p=0.0002, n=6 /$ group $)$ and $(I) R, R-H N K(F[5,30]=4.592, p=0.0031$, $n=6 /$ group) with TRKB. 
influence the TMD dimer conformation (Figure S3C). These findings are consistent with our experimental data showing an optimal CHOL concentration for TKRB function (Figure 1B), which is compromised as the TMD helices are separated at the $\mathrm{C}$ terminus at low $\mathrm{CHOL}$ concentration and adopt an unstable parallel TM orientation at high $\mathrm{CHOL}$ concentration (Figure $\mathrm{S} 3 \mathrm{~A}$ ).

Antidepressants bind to TRKB transmembrane domain Essentially all ADs promote TRKB signaling in rodents and this signaling is required for their behavioral effects (Castrén and Antila, 2017; Monteggia et al., 2004; Saarelainen et al., 2003). Many AD drugs are cationic amphipathic molecules that interact with phospholipids and accumulate at the lipid rafts (Chen et al., 2012; Erb et al., 2016; Kornhuber et al., 1995; Wray et al., 2019). We found that fluoxetine (FLX) and KET enhanced pTRKB at Y816 (Figure 2A) and FLX increased the surface expression of TRKB in primary cortical neurons (Figure 1F). FLX, imipramine, KET, and R,R-HNK increased TRKB interaction with PLC- $\gamma 1$ and their effects were blocked by $\beta C D X$ (Figures $1 \mathrm{C}$ and $\mathrm{S} 1 \mathrm{~N}-$ $S 1 Q)$, which indicates that $C H O L$ modulates $A D$-induced TRKB signaling. FLX partially rescued the reduction in BDNF-induced pTRKB response observed under high-CHOL (Figure 2B), suggesting that $A D$ s promote TRKB signaling particularly in synaptic-like membranes rich in $\mathrm{CHOL}$.

We then tested if ADs directly bind to TRKB. First, we found that biotinylated FLX binds to immunoprecipitated TRKB with a low $\mu \mathrm{M}$ affinity $\left(\mathrm{K}_{\mathrm{d}}=2.42 \mu \mathrm{M}\right)$ (Figure $2 \mathrm{C}$ ), but not to TRKA or lysates from non-transfected cells (Figures S4L and S4M). Although the affinity of FLX to serotonin transporter (5HTT) is much higher than that to TRKB, micromolar affinity corresponds well to the concentrations of $A D s$ reached in the human brain during chronic treatment (Bolo et al., 2000; Henry et al., 2000; Johnson et al., 2007; Karson et al., 1993). Binding of biotinylated FLX $(1 \mu \mathrm{M})$ to TRKB was displaced by unlabeled FLX $\left(K_{i}=\right.$ $1.69 \mu \mathrm{M})$ (Figure S4B), indicating specific binding. A deletion construct without most of the extracellular and intracellular domains of TRKB except for the TMD and short juxtamembrane sequences (TRKB.T1 1 EC) (Haapasalo et al., 1999) also demonstrated robust binding (Figure S4N), whereas FLX failed to bind to a chimeric TRKB with the TMD of TRKA (Figure 2D), which focuses the binding activity to the TRKB TMD.

Binding of FLX to TRKB was also observed in intact cells using in situ proximity ligation assay (PLA). A robust PLA signal was observed when N2A cells transfected to express TRKB were exposed to biotinylated FLX (Figures 2J and S5E-S5G), confirming a close proximity of bound FLX to TRKB. No signal was observed with FLX in control cells lacking TRKB (Figures $2 \mathrm{~K}$ and S5B-S5D).

To verify the direct interaction between FLX and TRKB, we used MST assay (Jerabek-Willemsen et al., 2014) that detects ligand-receptor binding directly in cell lysates (Welsch et al., 2017). This assay confirmed that unlabeled FLX directly binds to GFP-tagged TRKB in lysates of transfected HEK293T cells (Figure 2L).

We further found that tritiated imipramine binds to TRKB at micromolar affinity $\left(\mathrm{K}_{\mathrm{d}}=1.43 \mu \mathrm{M}\right)$ (Figure $2 \mathrm{~F}$ ), similar to that seen with FLX. Binding of biotinylated FLX $(1 \mu \mathrm{M})$ to TRKB was displaced by imipramine, venlafaxine, moclobemide, KET, esketamine, and $\mathrm{R}, \mathrm{R}-\mathrm{HNK}$ with $\mathrm{K}_{\mathrm{i}}$ of $1.03,2.08,1.51,12.30,2.86$, and $2.23 \mu \mathrm{M}$, respectively (Figures 2G and S4C-S4G). By contrast, control compounds isoproterenol, chlorpromazine, diphenhydramine, and $2 \mathrm{~S}, 6 \mathrm{~S}-\mathrm{HNK}$ that are structurally and physico-chemically similar to ADs, produced weak, if any, displacement of biotinylated FLX (Figures S4H and S4I). BDNF failed to displace FLX from TRKB (Figure S4J), which is consistent with different interaction sites.

The finding that KET and R,R-HNK compete with FLX indicates that not only the typical but also the novel rapid-acting ADs bind to TRKB. Remarkably, R, R-HNK clearly binds to TRKB $\left(K_{d}=1.82 \mu \mathrm{M}\right)$ (Figure $2 \mathrm{E}$ ), and S,S-HNK failed to displace bound $R, R,-H N K$, indicating that $A D$ binding to TRKB is stereoselective (Figure S4K). R,R-HNK produces AD-like effects in rodents at concentrations that do not inhibit NMDA receptors, the proposed primary interaction site for rapid-acting ADs (Zanos et al., 2016, 2018), but no alternative binding site for R,R-HNK that could explain its $A D$-like effects has been identified. Our finding suggests that TRKB might be this elusive direct target for R,R-HNK. CHOL did not compete with FLX or R,R-HNK, but increased the interaction of these compounds with TRKB, suggesting the presence of two distinct and cooperative recognition mechanisms for $\mathrm{CHOL}$ and ADs (Figures $2 \mathrm{H}$ and 2l).

These acute effects of ADs are not mediated by functional inhibition of acid sphingomyelinase (FIASMA) or sphingolipid metabolism (Kornhuber et al., 2010), because FIASMA compounds chlorpromazine, pimozide, and flupenthixol failed to rescue BDNF-induced activation of TRKB under high concentrations of $\mathrm{CHOL}$, as FLX does (Figures $2 \mathrm{~B}$ and $\mathrm{S} 2 \mathrm{H}$ ). Further, chlorpromazine failed to displace FLX binding to TRKB (Figure S4I), whereas non-FIASMA ADs venlafaxine, KET, and R,R-HNK readily displaced FLX (Figures S4E-S4G). Together, these results suggest that all of the investigated ADs directly bind to TRKB at clinically meaningful concentrations (Bolo et al., 2000; Henry et al., 2000; Karson et al., 1993).

\section{Modeling fluoxetine binding to TRKB}

Docking followed by an extensive set of $1201-\mu$ s-long MD simulations suggested a binding site and mode for FLX in the crevice facing the extracellular side of the crossed TRKB TMD dimer (Figure $3 \mathrm{~A}$ ). This binding site and mode engages both TMDs in the dimer and also recruits phospholipids, which can further stabilize the binding (Figure $3 \mathrm{~B}$ ). The simulations also revealed several protein residues important for binding, including $Y 433$, V437, and S440 (Figures 3A, 3C, and 3D). Mutagenesis experimentally verified this binding site: FLX binding to TRKB.Y433F

(J) In situ PLA demonstrates close proximity between biotinylated fluoxetine and TRKB on TRKB-expressing N2A cells (red dots).

(K) No PLA signal is seen in cells not expressing TRKB. Blue, DAPI; scale bar, $10 \mu \mathrm{m}$.

(L) MST demonstrated direct interaction between fluoxetine and GFP-tagged TRKB (15 min) in lysates from GFP-TRKB expressing HEK293T cells ( $\mathrm{n}=4 / \mathrm{group}$ ). Experimental traces depicted in the inset, vertical bars: blue, fluorescence cold; red, fluorescence hot.

See also Figures S2, S4, and S5. 
A

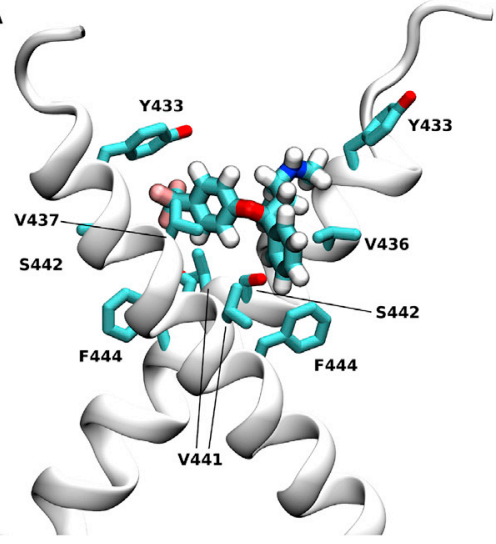

B

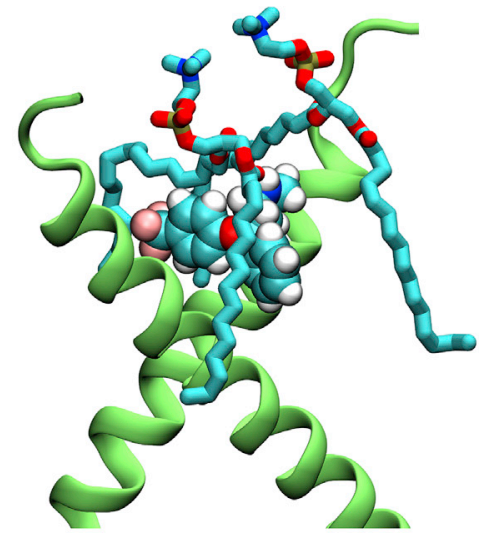

C

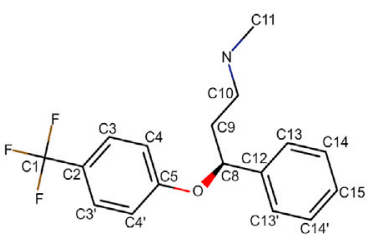

D

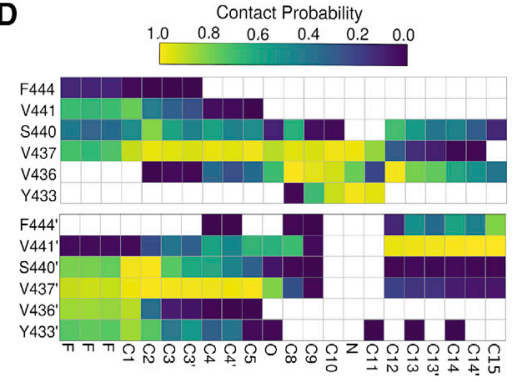

E

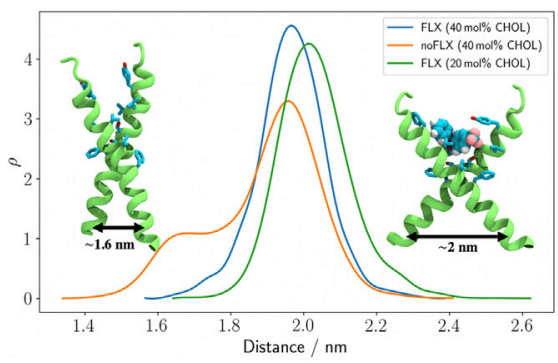

Figure 3. Model of fluoxetine interaction with TRKB transmembrane domain

The fluoxetine binding pocket at the dimeric interface of the TRKB transmembrane helices.

(A) A representative snapshot showing fluoxetine in the crevice between the TRKB monomers. Fluoxetine is shown in licorice and the protein in cartoon representations. The side chains that interact with the drug are labeled and shown in licorice.

(B) Fluoxetine binding involves lipid molecules, which provide a closed cavity for the drug. The protein is shown in green cartoon, the drug in van der Waals, and the lipids in licorice representations. (C) The chemical structure of fluoxetine. The atom names are labeled and the chemically equivalent atoms are indicated with an apostrophe.

(D) The contact probability between drug heavy atoms and the interacting protein residues. The upper and lower panels correspond to the two different transmembrane helices (residues of the second helix are tagged with an apostrophe). Contact probabilities are calculated using a minimum distance cutoff of $5 \AA$ (system 10).

(E) The distributions of the distance between the center of mass L451-L453 C $\alpha$ atoms of each monomer are shown for membranes with $20 \mathrm{~mol} \%$ cholesterol (green; system 9), $40 \mathrm{~mol} \%$ cholesterol with (blue; system 10) and without bound FLX (orange; system 7).

See also Figures S3 and S6 and Table S1.

decreased the FLX residence-time and binding affinity (Figure S4O). Together, these data suggest that FLX, by binding

and TRKB.V437A was essentially lost, and binding to TRKB.S440A was significantly reduced (Figures 2C and S4O). Furthermore, binding of FLX to a chimeric TRKB carrying TMD from TRKA (TRKB/TRKA.TM) was very low, and the affinity of imipramine and R,R-HNK to TRKB.Y433F was also much lower than to the wild-type TRKB (Figures 2D-2F).

As shown above, the stable configuration of TRKB TM dimers at $20 \mathrm{~mol} \%$ of $\mathrm{CHOL}$ is destabilized by increased membrane thickness at $40 \mathrm{~mol} \%$ of $\mathrm{CHOL}$ (Figure 1G,H). Remarkably, at $40 \mathrm{~mol} \%$, FLX binding maintained the active configuration of TRKB dimers close to that observed in 20 mol \% (Figure 3E), which is consistent with our biochemical observation that FLX preferentially acts under high $\mathrm{CHOL}$ conditions (Figure 2B). Following drug expulsion, the dimers transitioned to the more parallel conformation seen in Figure 1l. Protein-free simulations with varying $\mathrm{CHOL}$ concentrations showed that interaction of ADs with membrane lipids alone does not explain the observed drug binding, because neither FLX, R, R-HNK, nor esketamine altered the order parameters of the membrane lipids (systems 21-50 in Table S1). The Y433F mutation decreased the residence-time of FLX by at least 4-fold, to $161 \mathrm{~ns}$ when compared to the $>696 \mathrm{~ns}$ for the wild-type protein, consistent with a low binding affinity of FLX to TRKB.Y433F (Figure 2C). Accordingly, Y433 is directly involved in FLX binding and indirectly involved in $\mathrm{CHOL}$ sensing via membrane thickness. The other mutations (V437A, S440A; Table S1, systems 12-14) also substantially to the dimeric TRKB interface, acts like a wedge and stabilizes the cross-shaped active conformation at high $\mathrm{CHOL}$ concentration typically present in synaptic membranes (Figures $3 \mathrm{~A}$ and $3 \mathrm{E})$.

\section{Antidepressants promote membrane trafficking of TRKB}

We used fluorescence recovery after photobleaching (FRAP) assay in primary hippocampal neurons (DIV14) to evaluate the mobility of TRKB in neuronal spines. In neurons transfected to express GFP-tagged TRKB, the fluorescence was rapidly recovered in dendritic shafts, but not in spines after bleaching (Figures $4 \mathrm{~A}$ and S6A). Pretreatment with BDNF (20 ng/mL/15 min) brought about a rapid recovery of GFP-TRKB fluorescence in spines after bleaching, indicating TRKB trafficking to spines (Figures $4 \mathrm{~B}$ and $4 \mathrm{E})$. Similarly, pretreatment of neurons with FLX (1 $\mu \mathrm{M} / 15 \mathrm{~min})$ or KET (10 $\mu \mathrm{M} / 15 \mathrm{~min})$ also promoted recovery of GFP-TRKB fluorescence in dendritic spines (Figures 4C, 4D, $4 F, 4 G$, and S6A) without any additional effect on dendritic shafts. Neither BDNF, FLX, nor KET increased the fluorescence of GFP-TRKB.Y433F mutant receptors in dendritic spines after bleaching (Figures $4 \mathrm{H}-4 \mathrm{~J}$ ), although the localization of GFPTRKB.Y433F before bleaching was identical to the wild-type GFP-TRKB. These data demonstrate that BDNF, FLX, and KET promote TRKB trafficking in dendritic spines, and this effect is disrupted in TRKB.Y433F mutants. 


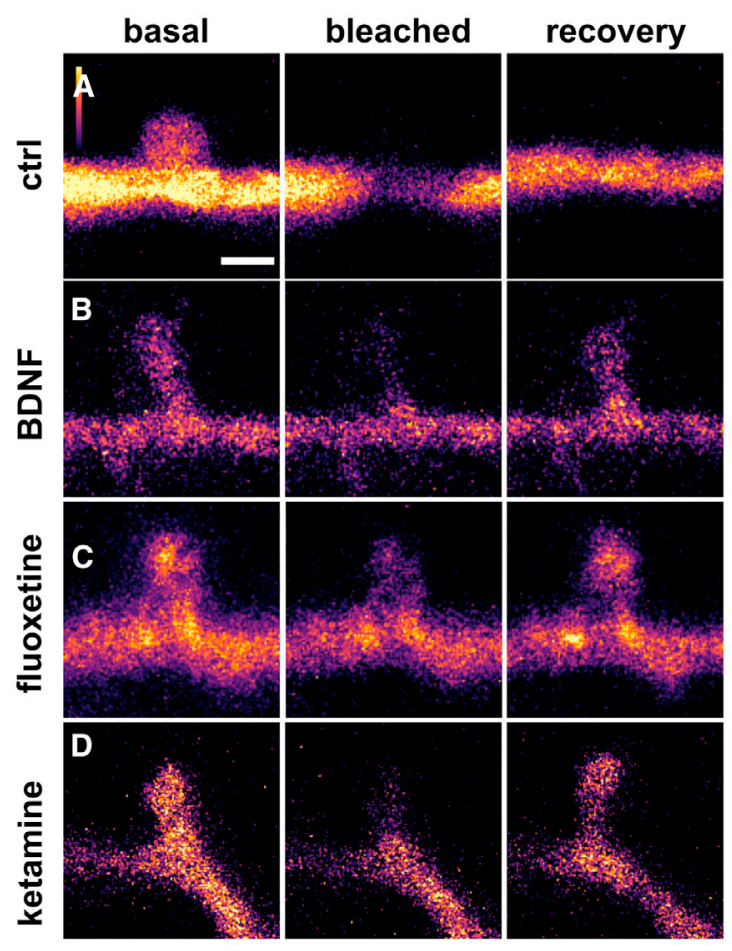

TRKB.wt
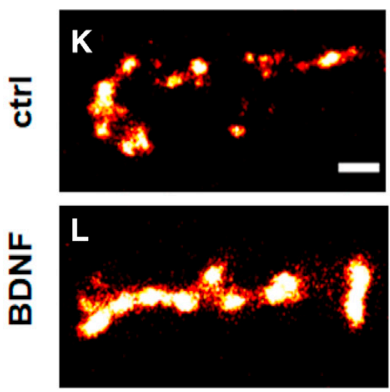

TRKB.Y433F
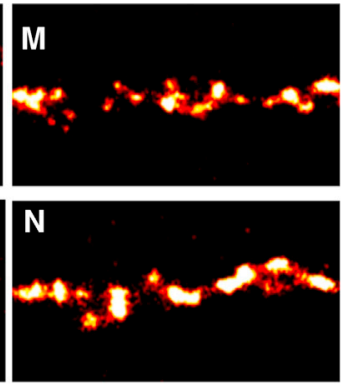

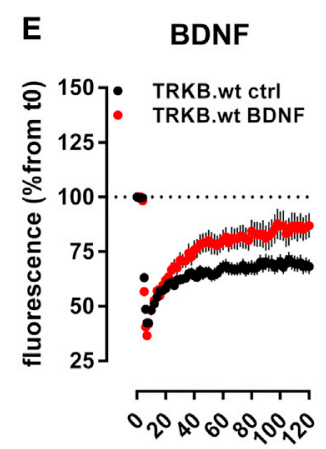

H
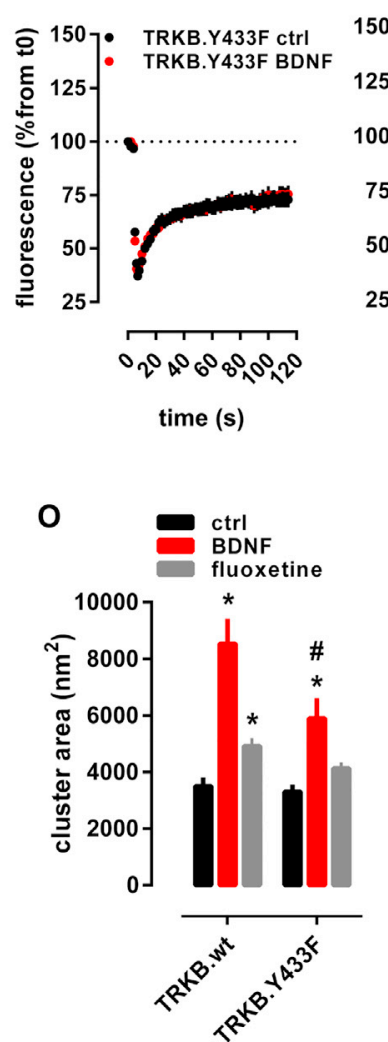

\section{F fluoxetine}

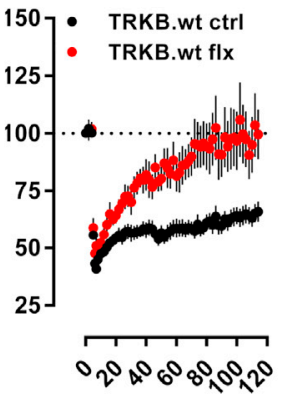

I
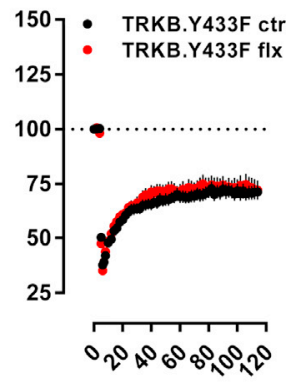

time (s)

\section{G ketamine}

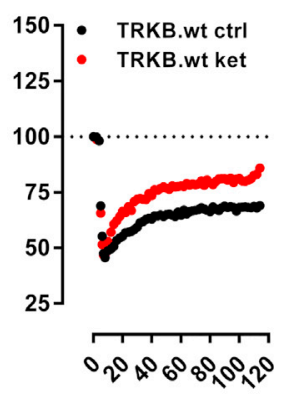

J

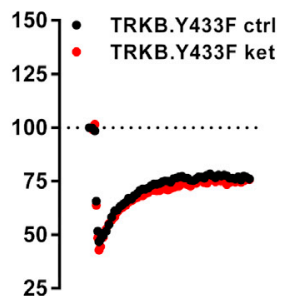

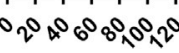

time (s)

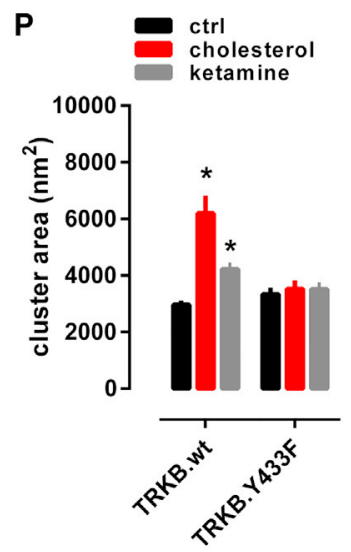

Figure 4. Antidepressants promote membrane trafficking of TRKB

(A-D) Representative images of the spine and shaft fluorescence in (A) control, (B) BDNF-, (C) fluoxetine-, or (D) ketamine-treated rat hippocampal neurons (E18; DIV14) transfected with GFP-TRKB before (basal), immediately (bleached), and $2 \mathrm{~min}$ (recovery) after photobleaching (for analysis of neurite shaft recovery, see Figure S4A). Scale bar, 1,000 $\mathrm{nm}$.

(E-J) Recovery of GFP-TRKB in dendritic spines is increased by $(E$ and $H)$ BDNF $(20 \mathrm{ng} / \mathrm{mL} / 15 \mathrm{~min}$, TRKB.wt $\mathrm{n}=17-27$; interaction: $\mathrm{F}[62,2,604]=5.435$, $p=0.0001$; TRKB.Y433F $n=27-39$; interaction: $F[52,3,328]=0.4595, p=0.99)$, $(F$ and $l)$ fluoxetine $(1 \mu \mathrm{M} / 15$ min, TRKB.wt $n=9-22$; interaction: $F[177,3,068]=$ 2.220, $p=0.0001$; TRKB.Y433F $n=28-42$; interaction: $F[59,4,012]=0.5555, p=0.99)$, and $(G$ and $J)$ ketamine $(10 \mu M / 15$ min, TRKB.wt $n=15-18$; interaction: $F[59,1,829]=3.361, p<0.0001$; TRKB.Y433F $n=20-22$; interaction: $F[59,2,360]=0.3995, p>0.9999)$, but this is prevented in GFP-TRKB.Y433F expressing neurons; data expressed as mean $\pm \mathrm{SEM}$ of percentage from $\mathrm{t}=0$.

(K-N) Representative images of the BDNF-induced clusters of GFP-TRKB on the surface of MG87.TRKB cells. Scale bar, $250 \mathrm{~nm}$.

$(\mathrm{O}$ and $\mathrm{P}) \mathrm{BDNF}(10 \mathrm{ng} / \mathrm{mL} / 15 \mathrm{~min})$ and fluoxetine $(10 \mu \mathrm{M} / 15 \mathrm{~min}$, TRKB.wt $\mathrm{n}=365-593 ;$ TRKB.Y433F $\mathrm{n}=232-547$; interaction: $\mathrm{F}[2,2,717]=4.305, \mathrm{p}=0.0136)(\mathrm{O})$ and cholesterol $(20 \mu \mathrm{M} / 15 \mathrm{~min})$ and ketamine $(10 \mu \mathrm{M} / 15 \mathrm{~min}$, TRKB. $w \mathrm{t} n=282-7,413$; TRKB. $Y 433 \mathrm{~F} n=258-765$; interaction: $F[2,2,731]=11.15, p<0.0001)(P)$ enhance the formation of clusters of GFP-TRKB on the surface of MG87.TRKB cells but not in the GFP-TRKB.Y433F-expressing cells. ${ }^{*} p<0.05$ from respective control (vehicletreated) groups; \#p < 0.05 from BDNF- or fluoxetine-treated wild-type group (Fisher's LSD), clusters from 10 cells/group, and 10 regions of interest (ROI) per image, mean \pm SEM of cluster area $\left(\mathrm{nm}^{2}\right)$.

See also Figure S6A. 
Super-resolution microscopy (dSTORM/TIRF) revealed that BDNF, FLX, KET, and CHOL all increased the size of clusters formed by wild-type GFP-TRKB, but not clusters of GFPTRKB.Y433F mutants at the plasma membrane of fibroblast cell line, indicating that the increased trafficking may lead to increased cell surface expression and clustering of TRKB (Figures 4K-4P). The basal cell surface expression of GFPTRKB.Y433F was again similar to that of the wild-type TRKB (Figures 4K, 4M, and 4O).

$A D s$ are known to increase the cell surface expression of AMPA glutamate receptors and the blockade of AMPA receptors prevents the behavioral effects of KET and R,R-HNK (Maeng et al., 2008; Zanos et al., 2016). We confirmed that FLX and R,R-HNK increased cell surface localization of GluR1 subunits of AMPA receptors, and this effect was prevented by the TRKB inhibitors ANA12 and k252a (Figures S6B and S6C) and in neurons from TRKB.Y433F mutant mice (Figures S6D and $\mathrm{S} 6 \mathrm{E})$. These data suggest that the effect of $A D s$ on synaptic AMPA receptor surface exposure is a downstream effect of TRKB activation by these drugs.

\section{Binding to TRKB mediates antidepressant-induced plasticity}

BDNF is a critical mediator of synaptic plasticity and is required for long-term potentiation (LTP) in slices as well as in vivo, and these effects are mediated by TRKB (Ernfors and Bramham, 2003; Minichiello, 2009; Panja and Bramham, 2014). Theta-burst stimulation reliably induced an LTP in the CA3-CA1 synapses in slices derived from wild-type mice. Remarkably, similar stimulation of slices derived from heterozygous mice carrying a TRKB.Y433F mutation (TRKB.Y433F mice) failed to induce any significant potentiation (Figure S6F). However, tetanic stimulation induced LTP in both wild-type and TRKB.Y433F slices (Figure S6G), consistent with the central role of BDNF in theta-burstmediated LTP (Kang et al., 1997; Minichiello et al., 2002; Patterson et al., 2001).

Infusion of BDNF into the dentate gyrus of anesthetized rats significantly increased synaptic strength, as previously reported (Messaoudi et al., 2002; Panja and Bramham, 2014). However, this effect of BDNF was partially prevented when rats were cotreated with pravastatin ( $10 \mathrm{mg} / \mathrm{kg} / \mathrm{day} / 14$ days) (Figure $5 \mathrm{~A}$ ), suggesting that neuronal $\mathrm{CHOL}$ is required for the effects of BDNF on LTP.

ADs increase the proliferation and survival of newly born dentate granule neurons (Malberg et al., 2000; Sairanen et al., 2005; Santarelli et al., 2003). We confirmed that FLX (15 mg/kg/day, leading to FLX brain concentration of $31.9 \pm 5.9 \mu \mathrm{M})$ (Table S2) significantly increased survival of newborn hippocampal neurons in wild-type mice, however, no increase was observed in the dentate gyrus of TRKB.Y433F mice (Figure 5B).

Chronic FLX treatment reactivates critical period-like plasticity in the visual cortex of adult mice, allowing an ocular dominance (OD) shift in response to monocular deprivation, which normally only happens during a developmental critical period (Maya Vetencourt et al., 2008; Steinzeig et al., 2017). In mice treated with FLX for 4 weeks (10 mg/kg/day), a 7-day monocular deprivation during the last treatment week induced a dramatic shift in OD in favor of the open eye (Figure 5C). We now show that both
KET and R,R-HNK (both $10 \mathrm{mg} / \mathrm{kg}$, intraperitoneal [i.p.]) also induced a significant shift in OD, but a much shorter treatment was needed than that for FLX (Figure $5 \mathrm{C}$ ), consistent with their fast action. The response to R,R-HNK was comparable to that produced by FLX, however, the magnitude of response to KET was lower than that to FLX and R,R-HNK. Remarkably, the effect of $\mathrm{FLX}$ and $\mathrm{R}, \mathrm{R}-\mathrm{HNK}$ on the shift in ocular dominance was lost in TRKB.Y433F mice (Figures 5D and 5E) and in wild-type mice cotreated with pravastatin (Figures $5 \mathrm{~F}$ and $5 \mathrm{G}$ ), indicating that the plasticity-inducing effects of ADs may be mediated by their direct binding to TRKB.

\section{Binding to TRKB mediates the behavioral effects of antidepressants}

We next investigated whether AD interaction with TRKB influences neuronal plasticity-dependent learning and behavior. FLX (15 mg/kg/day) for 7 days facilitated long-term memory in object location memory (OLM) test in TRKB.wt mice, but not in TRKB.Y433F mice, although the behavior of vehicle-treated TRKB.Y433F mice was similar to their vehicle-treated wild-type littermates (Figure 6A). A similar lack of response to FLX was observed in BDNF haploinsufficient mice (Figure S6J) and in animals co-treated with pravastatin (Figures 6B and 6C). Remarkably, serotonin transporter knockout (5HTT.ko) mice lacking the primary site of action of SSRIs responded to FLX treatment normally in the OLM test (Figure S6K), indicating that the effects of FLX in this test are not mediated by inhibition of serotonin transport. This is consistent with the findings that the biochemical, behavioral, and electrophysiological effects of SSRIs are preserved in 5HTT.ko mice (Normann et al., 2018; Rantamäki et al., 2011). However, a recent study found that behavioral effects of FLX are lost in mice with a point mutation in $5 \mathrm{HTT}$ that impairs the response to AD drugs (Nackenoff et al., 2016).

BDNF-TRKB signaling is known to be sufficient and necessary for the effects of ADs in the forced swimming test (FST) (Koponen et al., 2005; Monteggia et al., 2004; Saarelainen et al., 2003). FLX (15 mg/kg, for 21 days) and KET (10 mg/kg i.p., $2 \mathrm{~h}$ before) significantly reduced immobility in the FST in wild-type mice, but both drugs were ineffective in TRKB.Y433F mice (Figures 6D and 6E).

FLX promotes extinction of conditioned fear in a BDNFdependent manner (Andero and Ressler, 2012; Deschaux et al., 2011; Karpova et al., 2011). The freezing response was indistinguishable between the genotypes immediately after conditioning (Figures $6 \mathrm{G}$ and $6 \mathrm{H}$ ). FLX (15 mg/kg for 2 weeks, starting immediately after conditioning) and KET (10 mg/kg i.p., immediately after conditioning and $2 \mathrm{~h}$ before each extinction trial) promoted extinction of conditioned fear in wild-type mice (Figures $6 \mathrm{G}$ and $6 \mathrm{H}$ ), but no increase in fear extinction was seen in FLX- or KET-treated TRKB.Y433F mice (Figures 6G and $6 \mathrm{H}$ ) or in pravastatin-FLX co-treated mice (Figure 6F).

Together, these data demonstrate that the behavioral effects produced by different ADs were lost in TRKB.Y433F mutant mice, consistently with $A D$ binding to TRKB. Moreover, we observed that many of these behavioral effects were also lost when ADs were co-administered with pravastatin (Figures 6B, $6 \mathrm{C}$, and $6 \mathrm{~F}$ ), supporting the notion that $\mathrm{CHOL}$ sensing is necessary for the behavioral effects of BDNF on TRKB signaling. 

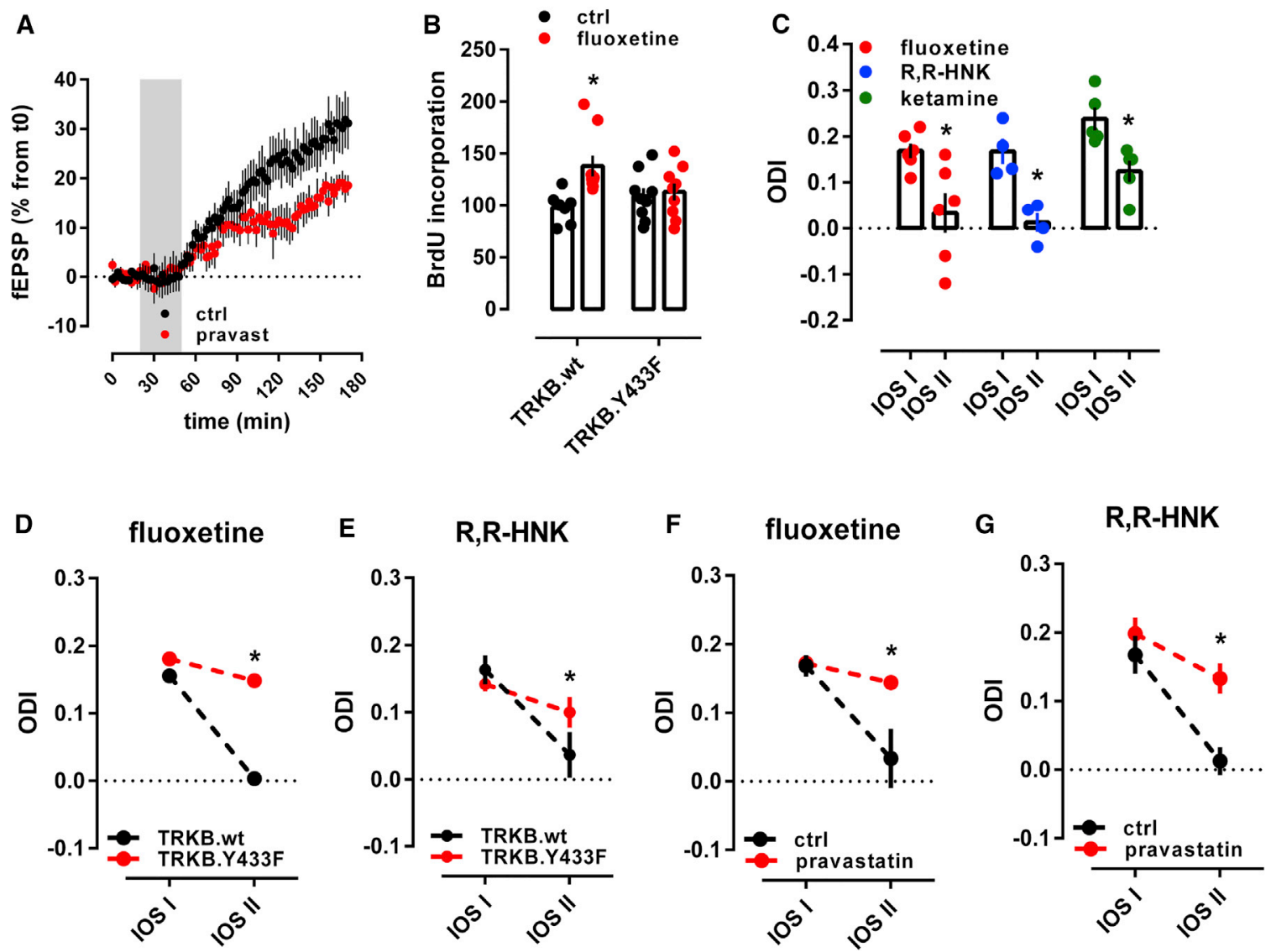

Figure 5. Binding to TRKB mediates the plasticity-related effects of antidepressants

(A) Treatment with pravastatin (10 $\mathrm{mg} / \mathrm{kg} /$ day in the drinking water for 14 days) attenuated the BDNF-induced LTP in the hippocampus of anesthetized rats $(\mathrm{F}[85,1,290]=1.484, \mathrm{p}=0.0036, \mathrm{n}=8-9)$.

(B) Fluoxetine promotes hippocampal neurogenesis in wild-type, but not in TRKB.Y433F mice $(n=7-9$; interaction: $F[1,30]=4.691, p=0.0384)$. Mice received bromodeoxyuridine (BrdU) injections at day 1, the BrdU incorporation was measured after 3 weeks of fluoxetine treatment $(15 \mathrm{mg} / \mathrm{kg} / \mathrm{day}$ for $21 \mathrm{days}$ in the drinking water, orally [p.o.]).

(C) Fluoxetine (10 mg/kg/day for 28 days, p.o.; $n=6)$, R,R-HNK (10 mg/kg i.p. injection every second day for 8 days, $n=4)$, and ketamine (10 mg/kg i.p. injection every second day for 8 days, $n=5$ ) permitted a shift in ocular dominance in adult mice during 7 days of monocular deprivation (paired $t$ test: fluoxetine: $t[5]=2.985$, $\mathrm{p}=0.0306$; R,R-HNK: $\mathrm{t}[3]=6.875, \mathrm{p}=0.0063$; ketamine: $\mathrm{t}[4]=6.517, \mathrm{p}=0.0029) .{ }^{*} \mathrm{p}<0.05$ between intrinsic signal imaging (IOS) sessions.

( $D$ and $E$ ) Fluoxetine $(D)$ and $R, R-H N K(E)$ fail to permit a shift in ocular dominance in TRKB.Y433F mice (fluoxetine: $F[1,19]=256.9, p<0.0001, n=9-12 ; R, R-H N K$ : $\mathrm{F}[1,20]=12.47, \mathrm{p}=0.0021, \mathrm{n}=6$ /group).

(F) Treatment with fluoxetine induced a shift in ocular dominance in response to 7 days of monocular deprivation, but this effect is prevented by pravastatin (interaction: $F[1,10]=5.221, p=0.0454, n=5-6$ ).

(G) R,R-HNK induced a shift in ocular dominance in response to 7 days of monocular deprivation, but this effect is prevented by pravastatin (treatment: $F[1,9]=$ 9.044; $p=0.0148, n=4-7)$. ${ }^{*} p<0.05$ from the control group in the same session, Fisher's LSD. Data expressed as mean \pm SEM. The black groups in plots $(F)$ and (G) are also depicted in (C).

See also Table S2 and Figure S6.

\section{DISCUSSION}

\section{Antidepressants bind to TRKB}

BDNF signaling is crucial for the action of essentially all AD drugs (Autry and Monteggia, 2012; Castrén and Antila, 2017; Duman and Monteggia, 2006), but this effect has been assumed to be indirectly mediated by other proteins such as $5 \mathrm{HTT}$ or NMDA receptors. We now show that ADs bind to the TMD of TRKB dimers with a therapeutically relevant affinity (Bolo et al., 2000; Karson et al., 1993), stabilizing a conformation of the TRKB TM dimers favorable for signaling, thereby promoting TRKB translocation to and reten- tion at the plasma membrane, where it is accessible to BDNF. Specific binding was observed not only for FLX and imipramine, representing typical SSRI and TCAs, respectively, but also for the rapid-acting KET metabolite RR-HNK. Binding of labeled FLX was displaced by FLX itself and by imipramine, moclobemide, RR-HNK, KET, and esketamine, which suggests that these drugs bind to at least partially overlapping sites. These data suggest that direct interaction with the TMDs of TRKB dimer may function as a binding site for several different, if not all, ADs.

MD simulations identified a binding site for FLX at the outer opening of the crossed dimer of TRKB TMDs. Several mutations 

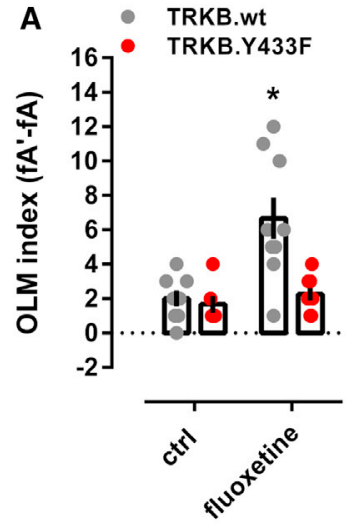

E

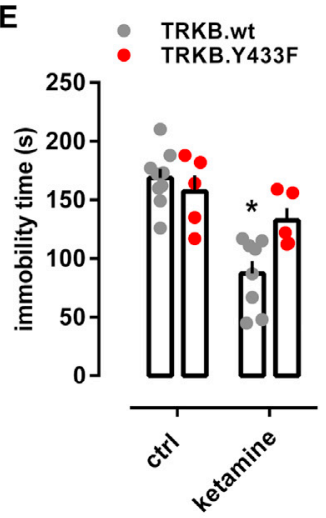

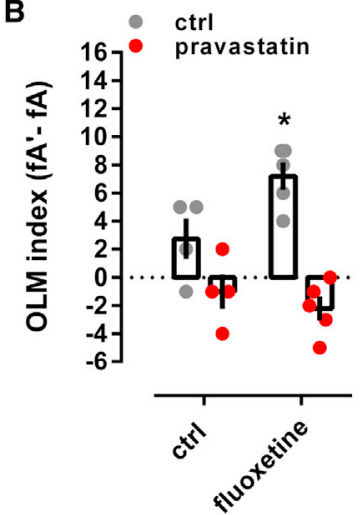
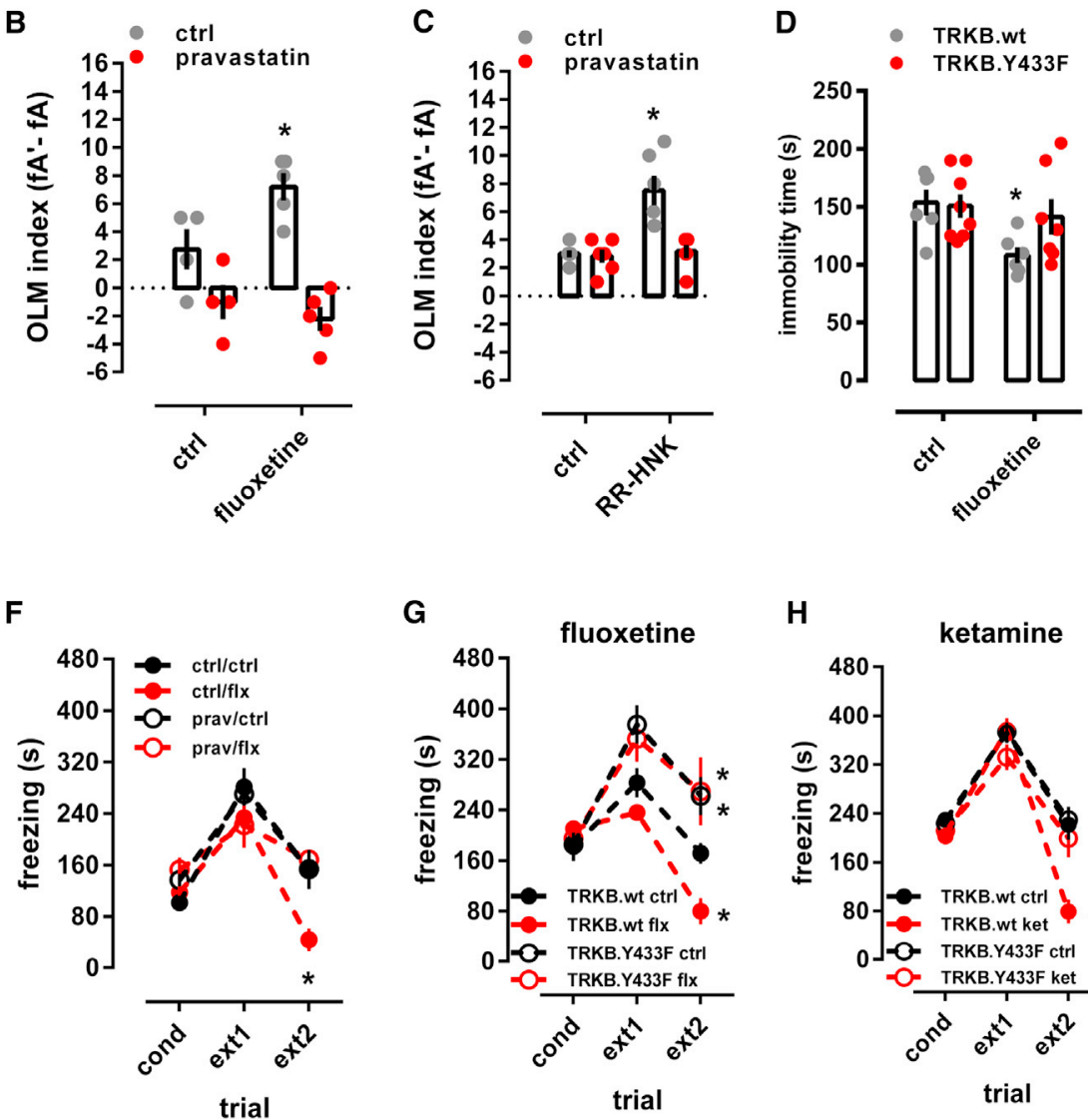

G

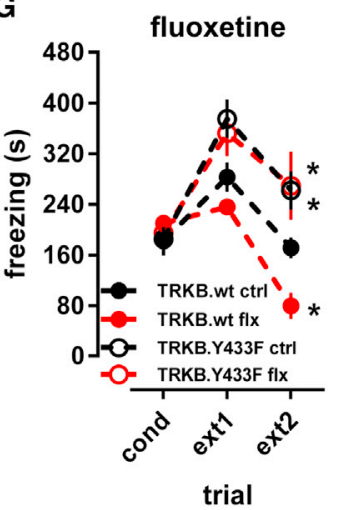

H

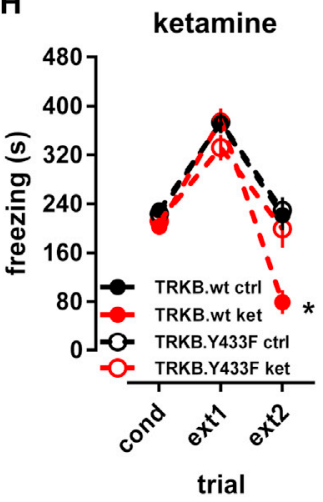

Figure 6. Binding to TRKB mediates the behavioral effects of antidepressants

(A) Fluoxetine improves object location memory (OLM) in wild-type mice, but this effect was absent in the TRKB.Y433F mice (interaction: F[1,18] = 6.878, $\mathrm{p}=0.017 ; \mathrm{n}=8-9)$.

(B) Fluoxetine improved object location memory in wild-type mice, but this effect was prevented by pravastatin (interaction: $F[1,14]=6.504, p=0.023, n=4-5$ ).

(C) R,R-HNK improved object location memory in wild-type mice, but this effect was prevented by pravastatin (interaction: $F[1,20]=10.59, p=0.0040$, $n=6$ /group).

(D and E) Fluoxetine (D) (treatment: $F[1,23]=5.433, p=0.0289, n=6-8$ ) and ketamine $(E)$ (treatment: $F[1,23]=24.26, p<0.0001, n=5-9)$ reduce immobility in the forced swimming test in TRKB.wt mice, but are ineffective in TRKB.Y433F mutants.

(F) Fluoxetine facilitated the extinction of contextual conditioned fear, and this response is blocked by pravastatin (interaction: $F[6,40]=5.099$, $p=0.0006$, $n=6$ /group)

$(\mathrm{G}$ and $\mathrm{H})$ Fluoxetine $(\mathrm{G})$ and ketamine $(\mathrm{H})$ facilitate the extinction of contextual conditioned fear in the 8-min session, and this response is blocked in mice carrying the TRKB.Y433F mutation (fluoxetine: $F[6,34]=3.241, p=0.0126 ; n=5-6$; ketamine: $F[6,40]=4.896, p=0.0008$; $n=5-7$ ). ${ }^{*} p<0.05$ from the control group in the same session, Fisher's LSD. Data expressed as mean \pm SEM.

See also Figure S6.

predicted by and tested in MD were used in experimental mutagenesis, which confirmed the binding site. Our data suggest that in thick CHOL-rich membranes, typically found in synapses and lipid rafts, dimers of TRKB TMDs assume a near-parallel, presumably unstable position, which leads to the exclusion of TRKB from synaptic membranes and limits synaptic TRKB signaling (Pereira and Chao, 2007; Suzuki et al., 2004). Binding of FLX to a site formed by the crossed TMDs acts as a wedge, maintaining a more stable structure in synaptic membranes, thereby allosterically facilitating synaptic BDNF signaling. Simulations predict that membrane lipids also participate in FLX binding to TRKB. Because TRKB exists as a multi-protein complex that also includes transmembrane proteins (Fred et al., 2019; Lesnikova et al., 2020), it is possible that other proteins and lipids participate in AD binding to TRKB in cell-type- and subcellular compartmentdependent manner. Further characterization of this binding site may yield important information for discovery of new ADs with increased potency for plasticity-related behavioral effects.

It has been known for decades that the clinical response to typical ADs is only reached after several days or weeks of treatment, but the reason for this delay has remained a mystery. One explanation has been that the process of neuronal plasticity induced by ADs may take time to develop. However, the discovery of the rapid action of KET, which is also dependent on plasticity, has undermined this explanation. FLX and imipramine bind to $5 \mathrm{HTT}$ with a much higher affinity than to TRKB, while the affinity of KET to TRKB is comparable to its affinity to NMDA receptors (Zanos et al., 2018). Remarkably, micromolar 
concentrations of typical ADs are reached and required in the brain during chronic treatment, as shown in humans for FLX (Bolo et al., 2000; Henry et al., 2000; Johnson et al., 2007; Karson et al., 1993), fluvoxamine (Bolo et al., 2000), and paroxetine (Henry et al., 2000) and here for FLX in mice. Importantly, typical ADs gradually accumulate in the brain, reaching a plateau after several weeks of treatment (Karson et al., 1993; Kornhuber et al., 1995), suggesting that the clinical response is only achieved when the drug reaches a brain concentration high enough to interact with a low-affinity binding target, such as TRKB. Sufficient concentrations may not be reached in fast metabolizers or patients with limited compliance, which may contribute to the failure to respond. KET, on the other hand, readily penetrates to the brain to achieve sufficient synaptic concentrations quickly. Therefore, the gradual increase in brain concentration to a level needed for TRKB binding might be at least one explanation for why typical ADs take so long to act, while the rapid brain penetration of KET enables fast action. Nevertheless, it is unlikely that effects on TRKB mediate all the effects of $A D s$ and that inhibition of 5HTT and NMDA receptors also play a role (Harmer et al., 2017).

A previous study reported binding of amitriptyline, but not many other ADs, to the extracellular domains of TRKB and TRKA (Jang et al., 2009). AD binding detected here is clearly distinct from that amitriptyline binding, as it includes many different $A D s$, it is specific to TRKB, and TRKB construct including the TMD but lacking the reported amitriptyline binding site in the first leucine-rich repeat readily binds FLX.

Our findings imply that high doses of statins might interfere with the $A D$ response. A recent study indicates more depression and more AD use among statin users (Köhler-Forsberg et al., 2019), but meta-analyses have found, if anything, less depression among statin users (Yatham et al., 2019). This discrepancy to our rodent findings is likely related to the high statin dose used in our studies. Interestingly, serum CHOL levels have been found to be low in suicidal patients (Kim and Myint, 2004).

Due to the effects of TRKB on neuronal survival and plasticity, small-molecule agonists of TRKB have been actively searched (Longo and Massa, 2013; Saragovi et al., 2019). Our data show that ADs bind to TRKB and allosterically potentiate BDNF signaling, thereby maintaining use-dependency, which limits the action of TRKB selectively to active synapses that release BDNF, avoiding undesirable stabilization of inactive synapses in a way full TRKB agonists may do. This action of ADs as "smart drugs" is consistent with the wide utility of these drugs in many neurological and psychiatric disorders beyond depression (Schneider et al., 2019).

\section{TRKB-cholesterol interaction}

Astrocyte-derived $\mathrm{CHOL}$ has been recognized as an important regulator of neuronal maturation and plasticity (Martin et al., 2014; Mauch et al., 2001; Pfrieger and Ungerer, 2011), but the mechanisms through which $\mathrm{CHOL}$ acts to produce these effects have remained unclear. Here, we have demonstrated that TRKB TMD possesses a CARC domain (Fantini and Barrantes, 2013), and $\mathrm{CHOL}$ potentiates the effects of BDNF on TRKB signaling.

$\mathrm{CHOL}$ regulates BDNF signaling (Pereira and Chao, 2007; Suzuki et al., 2004; Zonta and Minichiello, 2013), and BDNF, in turn, promotes neuronal CHOL synthesis (Suzuki et al., 2007). Synap- tic membranes are enriched in $\mathrm{CHOL}$ and resemble $\mathrm{CHOL}$-rich lipid rafts (Ikonen, 2008). TRKB normally resides outside rafts but can transiently translocate to rafts upon BDNF stimulation (Pereira and Chao, 2007; Suzuki et al., 2004), as also observed here. This translocation may be related to our observation of TRKB trafficking to dendritic spines and clustering on the plasma membrane, both of which were stimulated by BDNF and ADs. TRKB residence in lipid rafts is short-lived (Pereira and Chao, 2007; Suzuki et al., 2004), which may be explained by our simulation data suggesting instability of the crossed TRKB TMD structure in thick $\mathrm{CHOL}$-rich membranes. Translocation of TRKB to rafts is dependent on FYN kinase (Pereira and Chao, 2007; Suzuki et al., 2004), and we observed that BDNF increases interaction of FYN with wild-type TRKB, but not with the TRKB.Y433F mutant cells. These data suggest a scenario where the interaction between TRKB and BDNF or ADs promotes its retention in $\mathrm{CHOL}$-rich synaptic membranes.

Our simulation data predict that TMDs of TRKB interact at

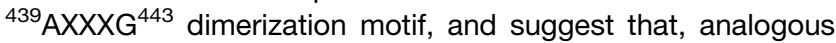
to the EGF receptor (Arkhipov et al., 2013; Endres et al., 2013; Sinclair et al., 2018), the angle between the dimerized and crossed TRKB TMDs, regulated by the CHOL-regulated membrane thickness, plays an important role in TRKB signaling. Obviously, the configuration of the TMD is not the only determinant of TRKB signaling capacity, nevertheless, our findings are a major step forward in understanding the interaction of TRKB with cellular membranes.

TRKB appears to be the only CHOL-sensing member from the TRK family of neurotrophin receptors. Although TRK family members show high homology, the TMD of TRKB differs from that of TRKA and TRKC, which, in contrast to TRKB, act as dependence receptors inducing cell death in the absence of a ligand (Nikoletopoulou et al., 2010); this property is apparently dependent on the transmembrane domain (Dekkers et al., 2013). Our data suggest that TRKB has evolved to become a CHOL sensor, which may be important for its function as mediator of activity-dependent plasticity.

\section{Conclusions}

The present findings demonstrate that ADs bind to TRKB and allosterically increase BDNF signaling, thereby directly linking the effects of ADs to neuronal plasticity. AD-induced plasticity is utilized by network-specific neuronal activity to guide re-wiring of plastic networks, allowing beneficial re-adaptation of networks abnormally wired during development or by stress (Castrén, 2013). Our data suggest a framework that unites the effects of all ADs with therapy-mediated guidance to achieve the clinical $A D$ response.

\section{STAR $\star$ METHODS}

Detailed methods are provided in the online version of this paper and include the following:

- KEY RESOURCES TABLE

- RESOURCE AVAILABILITY

O Lead contact

O Materials availability

○ Data and code availability 


\section{- EXPERIMENTAL MODEL AND SUBJECT DETAILS ○ Cell culture \\ O Animals \\ Pronucleus injections \\ O Founder analysis \\ - METHOD DETAILS \\ O In silico methods \\ O In vitro methods \\ O In vivo methods \\ - QUANTIFICATION AND STATISTICAL ANALYSIS}

\section{SUPPLEMENTAL INFORMATION}

Supplemental Information can be found online at https://doi.org/10.1016/j. cell.2021.01.034.

\section{ACKNOWLEDGMENTS}

The authors thank Sulo Kolehmainen and Seija Lågas (Neuroscience CenterUH), Marc Baumann and Rabah Soliymani (Department of Biochemistry and Developmental Biology-UH), and the Biomedicum Imaging Unit (BIU) at the University of Helsinki for technical help. We also thank Drs. Todd Gould (U. of Maryland) and Craig Thomas (NIH) for kindly supplying the R,R-HNK, Henri Huttunen (UH) for supplying GLuc-coupled raft-restricted FYN construct, and Yves-Alain Barde, Maija Castrén, Juan Lima-Ojeda, and Heikki Ruskoaho for their insightful comments to the manuscript. The E.C. lab was supported by the ERC (322742-iPLASTICITY), JPND CircProt (project 301225 and project 643417), the Sigrid Jusélius Foundation, the Jane and Aatos Erkko Foundation, and the Academy of Finland (294710 and 307416). C.R.A.F.D. received grants from FAPESP (project 2018/18500-3 and project 2018/04250-5). The C.R.B. lab was supported by the JPND CircProt (project 30122). The S.E.L. lab was funded by the Academy of Finland (297211). The I.V. lab was supported by the Academy of Finland (Center of Excellence) (307415), the Sigrid Juselius Foundation, the Helsinki Institute of Life Science, Human Frontier Science Program (project RGP0059/2019), and CSC-IT Center for Science. T.S. was funded by the German Research Council (SE 2666/2-1) and the Forschungskommission of the Medical Faculty of the University of Freiburg (SER1149/17). The M.S. lab was funded by the Jane and Aatos Erkko Foundation. None of the funders had a role in the data acquisition and analysis or manuscript preparation.

\section{AUTHOR CONTRIBUTIONS}

P.C.C., C.B., I.V., and E.C. designed the experiments. P.C.C., S.M.F., C.A.B., V.K., M.P.S., K.K., and C.B. performed the biochemical experiments with the assistance of L.L. and I.C. M.G., G.E., T.R., and I.V. performed the molecular modeling and simulation experiments. C.B., S.M.F., R.M., and C.A.B. performed the imaging experiments. C.C., H.A., and A.S. performed intrinsic optical imaging experiments. F.W., S.P., and S.E.L. performed the electrophysiology experiments. P.C.C., C.R.A.F.D., C.C., S.V., and T.S. performed the behavioral studies. P.C.C. and E.C. wrote the manuscript, with the help of M.G., G.E., T.R., C.R.B., C.N., M.S., and I.V.

\section{DECLARATION OF INTERESTS}

E.C. and M.S. are shareholders of Herantis Pharma Plc that is not related to this study. E.C. has received lecture fees from Janssen-Cilag. Other authors declare no conflicts of interest.

Received: February 26, 2020

Revised: December 22, 2020

Accepted: January 21, 2021

Published: February 18, 2021

\section{REFERENCES}

Abdallah, C.G., Sanacora, G., Duman, R.S., and Krystal, J.H. (2015). Ketamine and rapid-acting antidepressants: a window into a new neurobiology for mood disorder therapeutics. Annu. Rev. Med. 66, 509-523.

Abraham, M.J., Murtola, T., Schulz, R., Páll, S., Smith, J.C., Hess, B., and Lindahl, E. (2015). GROMACS: high performance molecular simulations through multi-level parallelism from laptops to supercomputers. SoftwareX 1-2, 19-25.

Alford, R.F., Koehler Leman, J., Weitzner, B.D., Duran, A.M., Tilley, D.C., Elazar, A., and Gray, J.J. (2015). An Integrated Framework Advancing Membrane Protein Modeling and Design. PLoS Comput. Biol. 11, e1004398.

Ampuero, E., Stehberg, J., Gonzalez, D., Besser, N., Ferrero, M., Diaz-Veliz, G., Wyneken, U., and Rubio, F.J. (2013). Repetitive fluoxetine treatment affects long-term memories but not learning. Behav. Brain Res. 247, 92-100.

Andero, R., and Ressler, K.J. (2012). Fear extinction and BDNF: translating animal models of PTSD to the clinic. Genes Brain Behav. 11, 503-512.

Antila, H., Autio, H., Turunen, L., Harju, K., Tammela, P., Wennerberg, K., YliKauhaluoma, J., Huttunen, H.J., Castrén, E., and Rantamäki, T. (2014). Utilization of in situ ELISA method for examining Trk receptor phosphorylation in cultured cells. J. Neurosci. Methods 222, 142-146.

Arkhipov, A., Shan, Y., Das, R., Endres, N.F., Eastwood, M.P., Wemmer, D.E., Kuriyan, J., and Shaw, D.E. (2013). Architecture and membrane interactions of the EGF receptor. Cell 152, 557-569.

Autry, A.E., and Monteggia, L.M. (2012). Brain-derived neurotrophic factor and neuropsychiatric disorders. Pharmacol. Rev. 64, 238-258.

Baeza-Raja, B., Sachs, B.D., Li, P., Christian, F., Vagena, E., Davalos, D., Le Moan, N., Ryu, J.K., Sikorski, S.L., Chan, J.P., et al. (2016). p75 Neurotrophin Receptor Regulates Energy Balance in Obesity. Cell Rep. 14, 255-268.

Beglov, D., and Roux, B. (1994). Finite representation of an infinite bulk system: Solvent boundary potential for computer simulations. J. Chem. Phys. 100 9050-9063.

Belmaker, R.H., and Agam, G. (2008). Major depressive disorder. N. Engl. J. Med. 358, 55-68.

Berendsen, H.J.C., Postma, J.P.M., van Gunsteren, W.F., DiNola, A., and Haak, J.R. (1984). Molecular dynamics with coupling to an external bath. J. Chem. Phys. 81, 3684-3690.

Berman, R.M., Cappiello, A., Anand, A., Oren, D.A., Heninger, G.R., Charney, D.S., and Krystal, J.H. (2000). Antidepressant effects of ketamine in depressed patients. Biol. Psychiatry 47, 351-354.

Beutler, T.C., Mark, A.E., van Schaik, R.C., Gerber, P.R., and van Gunsteren, W.F. (1994). Avoiding singularities and numerical instabilities in free energy calculations based on molecular simulations. Chem. Phys. Lett. 222, 529-539.

Bolo, N.R., Hodé, Y., Nédélec, J.F., Lainé, E., Wagner, G., and Macher, J.P. (2000). Brain pharmacokinetics and tissue distribution in vivo of fluvoxamine and fluoxetine by fluorine magnetic resonance spectroscopy. Neuropsychopharmacology 23, 428-438.

Cang, J., Rentería, R.C., Kaneko, M., Liu, X., Copenhagen, D.R., and Stryker, M.P. (2005). Development of precise maps in visual cortex requires patterned spontaneous activity in the retina. Neuron 48, 797-809.

Castrén, E. (2013). Neuronal network plasticity and recovery from depression. JAMA Psychiatry 70, 983-989.

Castrén, E., and Antila, H. (2017). Neuronal plasticity and neurotrophic factors in drug responses. Mol. Psychiatry 22, 1085-1095.

Chen, J., Korostyshevsky, D., Lee, S., and Perlstein, E.O. (2012). Accumulation of an antidepressant in vesiculogenic membranes of yeast cells triggers autophagy. PLoS ONE 7, e34024.

Das, I., Krzyzosiak, A., Schneider, K., Wrabetz, L., D’Antonio, M., Barry, N., Sigurdardottir, A., and Bertolotti, A. (2015). Preventing proteostasis diseases by selective inhibition of a phosphatase regulatory subunit. Science 348 , 239-242. 
Dekkers, M.P.J., Nikoletopoulou, V., and Barde, Y.-A. (2013). Cell biology in neuroscience: Death of developing neurons: new insights and implications for connectivity. J. Cell Biol. 203, 385-393.

Dere, E., Huston, J.P., and De Souza Silva, M.A. (2007). The pharmacology, neuroanatomy and neurogenetics of one-trial object recognition in rodents. Neurosci. Biobehav. Rev. 31, 673-704.

Deschaux, O., Spennato, G., Moreau, J.-L., and Garcia, R. (2011). Chronic treatment with fluoxetine prevents the return of extinguished auditory-cued conditioned fear. Psychopharmacology (Berl.) 215, 231-237.

Di Lieto, A., Rantamäki, T., Vesa, L., Yanpallewar, S., Antila, H., Lindholm, J., Rios, M., Tessarollo, L., and Castrén, E. (2012). The responsiveness of TrkB to BDNF and antidepressant drugs is differentially regulated during mouse development. PLoS ONE 7, e32869.

Diniz, C.R.A.F., Casarotto, P.C., Fred, S.M., Biojone, C., Castrén, E., and Joca, S.R.L. (2018). Antidepressant-like effect of losartan involves TRKB transactivation from angiotensin receptor type 2 (AGTR2) and recruitment of FYN. Neuropharmacology 135, 163-171.

Du, J., Feng, L., Yang, F., and Lu, B. (2000). Activity- and Ca(2+)-dependent modulation of surface expression of brain-derived neurotrophic factor receptors in hippocampal neurons. J. Cell Biol. 150, 1423-1434.

Duman, R.S., and Monteggia, L.M. (2006). A neurotrophic model for stressrelated mood disorders. Biol. Psychiatry 59, 1116-1127.

Endres, N.F., Das, R., Smith, A.W., Arkhipov, A., Kovacs, E., Huang, Y., Pelton, J.G., Shan, Y., Shaw, D.E., Wemmer, D.E., et al. (2013). Conformational coupling across the plasma membrane in activation of the EGF receptor. Cell 152, 543-556.

Erb, S.J., Schappi, J.M., and Rasenick, M.M. (2016). Antidepressants Accumulate in Lipid Rafts Independent of Monoamine Transporters to Modulate Redistribution of the G Protein, Gas. J. Biol. Chem. 291, 19725-19733.

Ernfors, P., and Bramham, C.R. (2003). The coupling of a trkB tyrosine residue to LTP. Trends Neurosci. 26, 171-173.

Essmann, U., Perera, L., Berkowitz, M.L., Darden, T., Lee, H., and Pedersen, L.G. (1995). A smooth particle mesh Ewald method. J. Chem. Phys. 103, 8577-8593.

Fantini, J., and Barrantes, F.J. (2013). How cholesterol interacts with membrane proteins: an exploration of cholesterol-binding sites including CRAC, CARC, and tilted domains. Front. Physiol. 4, 31.

Fantini, J., Epand, R.M., and Barrantes, F.J. (2019). Cholesterol-Recognition Motifs in Membrane Proteins. Adv. Exp. Med. Biol. 1135, 3-25.

Fleishman, S.J., Leaver-Fay, A., Corn, J.E., Strauch, E.-M., Khare, S.D., Koga, N., Ashworth, J., Murphy, P., Richter, F., Lemmon, G., et al. (2011). RosettaScripts: a scripting language interface to the Rosetta macromolecular modeling suite. PLoS ONE 6, e20161.

Fred, S.M., Laukkanen, L., Brunello, C.A., Vesa, L., Göös, H., Cardon, I., Moliner, R., Maritzen, T., Varjosalo, M., Casarotto, P.C., and Castrén, E. (2019). Pharmacologically diverse antidepressants facilitate TRKB receptor activation by disrupting its interaction with the endocytic adaptor complex AP-2. J. Biol. Chem. 294, 18150-18161.

González-González, I.M., Jaskolski, F., Goldberg, Y., Ashby, M.C., and Henley, J.M. (2012). Measuring membrane protein dynamics in neurons using fluorescence recovery after photobleach. Methods Enzymol. 504, 127-146.

Greifzu, F., Pielecka-Fortuna, J., Kalogeraki, E., Krempler, K., Favaro, P.D., Schlüter, O.M., and Löwel, S. (2014). Environmental enrichment extends ocular dominance plasticity into adulthood and protects from stroke-induced impairments of plasticity. Proc. Natl. Acad. Sci. USA 111, 1150-1155.

Haapasalo, A., Saarelainen, T., Moshnyakov, M., Arumäe, U., Kiema, T.R., Saarma, M., Wong, G., and Castrén, E. (1999). Expression of the naturally occurring truncated trkB neurotrophin receptor induces outgrowth of filopodia and processes in neuroblastoma cells. Oncogene 18, 1285-1296.

Haapasalo, A., Sipola, I., Larsson, K., Akerman, K.E.O., Stoilov, P., Stamm, S., Wong, G., and Castren, E. (2002). Regulation of TRKB surface expression by brain-derived neurotrophic factor and truncated TRKB isoforms. J. Biol. Chem. 277, 43160-43167.
Harmer, C.J., Duman, R.S., and Cowen, P.J. (2017). How do antidepressants work? New perspectives for refining future treatment approaches. Lancet Psychiatry 4, 409-418.

Henry, M.E., Moore, C.M., Kaufman, M.J., Michelson, D., Schmidt, M.E., Stoddard, E., Vuckevic, A.J., Berreira, P.J., Cohen, B.M., and Renshaw, P.F. (2000). Brain kinetics of paroxetine and fluoxetine on the third day of placebo substitution: a fluorine MRS study. Am. J. Psychiatry 157, 1506-1508.

Hess, B., Bekker, H., Berendsen, H.J.C., and Fraaije, J.G.E.M. (1997). LINCS: a linear constraint solver for molecular simulations. J. Comput. Chem. 18, 1463-1472.

Hoover, W.G. (1985). Canonical dynamics: Equilibrium phase-space distributions. Phys. Rev. A Gen. Phys. 31, 1695-1697.

Huang, J., Rauscher, S., Nawrocki, G., Ran, T., Feig, M., de Groot, B.L., Grubmüller, H., and MacKerell, A.D., Jr. (2017). CHARMM36m: an improved force field for folded and intrinsically disordered proteins. Nat. Methods 14, 71-73.

Ikonen, E. (2008). Cellular cholesterol trafficking and compartmentalization. Nat. Rev. Mol. Cell Biol. 9, 125-138.

Jacobson, K., Mouritsen, O.G., and Anderson, R.G.W. (2007). Lipid rafts: at a crossroad between cell biology and physics. Nat. Cell Biol. 9, 7-14.

Jang, S.-W., Liu, X., Chan, C.-B., Weinshenker, D., Hall, R.A., Xiao, G., and Ye, K. (2009). Amitriptyline is a TrkA and TrkB receptor agonist that promotes TrkA TrkB heterodimerization and has potent neurotrophic activity. Chem. Biol. 16, 644-656.

Jerabek-Willemsen, M., André, T., Wanner, R., Roth, H.M., Duhr, S., Baaske, P., and Breitsprecher, D. (2014). MicroScale Thermophoresis: Interaction analysis and beyond. J. Mol. Struct. 1077, 101-113.

Jiang, W., and Roux, B. (2010). Free Energy Perturbation Hamiltonian ReplicaExchange Molecular Dynamics (FEP/H-REMD) for Absolute Ligand Binding Free Energy Calculations. J. Chem. Theory Comput. 6, 2559-2565.

Jo, S., Cheng, X., Lee, J., Kim, S., Park, S.-J., Patel, D.S., Beaven, A.H., Lee, K.I., Rui, H., Park, S., et al. (2017). CHARMM-GUI 10 years for biomolecular modeling and simulation. J. Comput. Chem. 38, 1114-1124.

Johnson, R.D., Lewis, R.J., and Angier, M.K. (2007). The distribution of fluoxetine in human fluids and tissues. J. Anal. Toxicol. 31, 409-414.

Jorgensen, W.L., Chandrasekhar, J., Madura, J.D., Impey, R.W., and Klein, M.L. (1983). Comparison of simple potential functions for simulating liquid water. J. Chem. Phys. 79, 926-935.

Kalatsky, V.A., and Stryker, M.P. (2003). New paradigm for optical imaging: temporally encoded maps of intrinsic signal. Neuron 38, 529-545.

Kang, H., Welcher, A.A., Shelton, D., and Schuman, E.M. (1997). Neurotrophins and time: different roles for TrkB signaling in hippocampal long-term potentiation. Neuron 19, 653-664.

Karpova, N.N., Pickenhagen, A., Lindholm, J., Tiraboschi, E., Kulesskaya, N., Agústsdóttir, A., Antila, H., Popova, D., Akamine, Y., Bahi, A., et al. (2011). Fear erasure in mice requires synergy between antidepressant drugs and extinction training. Science 334, 1731-1734.

Karson, C.N., Newton, J.E., Livingston, R., Jolly, J.B., Cooper, T.B., Sprigg, J., and Komoroski, R.A. (1993). Human brain fluoxetine concentrations. J. Neuropsychiatry Clin. Neurosci. 5, 322-329.

Kim, Y.-K., and Myint, A.-M. (2004). Clinical application of low serum cholesterol as an indicator for suicide risk in major depression. J. Affect. Disord. $81,161-166$.

Klauda, J.B., Venable, R.M., Freites, J.A., O'Connor, J.W., Tobias, D.J., Mondragon-Ramirez, C., Vorobyov, I., MacKerell, A.D., Jr., and Pastor, R.W. (2010). Update of the CHARMM all-atom additive force field for lipids: validation on six lipid types. J. Phys. Chem. B 114, 7830-7843.

Klimovich, P.V., Shirts, M.R., and Mobley, D.L. (2015). Guidelines for the analysis of free energy calculations. J. Comput. Aided Mol. Des. 29, 397-411.

Köhler-Forsberg, O., Gasse, C., Petersen, L., Nierenberg, A.A., Mors, O., and Østergaard, S.D. (2019). Statin treatment and the risk of depression. J. Affect. Disord. 246, 706-715. 
Koponen, E., Rantamäki, T., Voikar, V., Saarelainen, T., MacDonald, E., and Castrén, E. (2005). Enhanced BDNF signaling is associated with an antidepressant-like behavioral response and changes in brain monoamines. Cell. Mol. Neurobiol. 25, 973-980.

Kornhuber, J., Retz, W., and Riederer, P. (1995). Slow accumulation of psychotropic substances in the human brain. Relationship to therapeutic latency of neuroleptic and antidepressant drugs? J. Neural Transm. Suppl. 46, 315-323.

Kornhuber, J., Tripal, P., Reichel, M., Mühle, C., Rhein, C., Muehlbacher, M., Groemer, T.W., and Gulbins, E. (2010). Functional Inhibitors of Acid Sphingomyelinase (FIASMAs): a novel pharmacological group of drugs with broad clinical applications. Cell. Physiol. Biochem. 26, 9-20.

Koskinen, M., Bertling, E., and Hotulainen, P. (2012). Chapter three - Methods to Measure Actin Treadmilling Rate in Dendritic Spines. In Methods in Enzymology, P.M. Conn, ed. (Academic Press), pp. 47-58.

Lesnikova, A., Casarotto, P.C., Fred, S.M., Voipio, M., Winkel, F., Steinzeig, A., Antila, H., Umemori, J., Biojone, C., and Castrén, E. (2020). Chondroitinase and antidepressants promote plasticity by releasing TRKB from dephosphorylating control of PTP $\sigma$ in parvalbumin neurons. J. Neurosci. Published online December 8, 2020. https://doi.org/10.1523/JNEUROSCI.2228-20.2020.

Li, S.C., and Ng, Y.K. (2010). Calibur: a tool for clustering large numbers of protein decoys. BMC Bioinformatics 11, 25.

Li, E., Wimley, W.C., and Hristova, K. (2012). Transmembrane helix dimerization: beyond the search for sequence motifs. Biochim. Biophys. Acta 1818, 183-193.

Lomize, M.A., Lomize, A.L., Pogozheva, I.D., and Mosberg, H.I. (2006). OPM: orientations of proteins in membranes database. Bioinformatics 22, 623-625.

Lomize, A.L., Lomize, M.A., Krolicki, S.R., and Pogozheva, I.D. (2017). Membranome: a database for proteome-wide analysis of single-pass membrane proteins. Nucleic Acids Res. 45 (D1), D250-D255.

Lomize, A.L., Hage, J.M., and Pogozheva, I.D. (2018). Membranome 2.0: database for proteome-wide profiling of bitopic proteins and their dimers. Bioinformatics 34, 1061-1062.

Longo, F.M., and Massa, S.M. (2013). Small-molecule modulation of neurotrophin receptors: a strategy for the treatment of neurological disease. Nat. Rev. Drug Discov. 12, 507-525.

Maeng, S., Zarate, C.A., Jr., Du, J., Schloesser, R.J., McCammon, J., Chen, G., and Manji, H.K. (2008). Cellular mechanisms underlying the antidepressant effects of ketamine: role of alpha-amino-3-hydroxy-5-methylisoxazole-4-propionic acid receptors. Biol. Psychiatry 63, 349-352.

Malberg, J.E., Eisch, A.J., Nestler, E.J., and Duman, R.S. (2000). Chronic antidepressant treatment increases neurogenesis in adult rat hippocampus. J. Neurosci. 20, 9104-9110.

Malhi, G.S., and Mann, J.J. (2018). Depression. Lancet 392, 2299-2312.

Martin, M.G., Ahmed, T., Korovaichuk, A., Venero, C., Menchón, S.A., Salas, I., Munck, S., Herreras, O., Balschun, D., and Dotti, C.G. (2014). Constitutive hippocampal cholesterol loss underlies poor cognition in old rodents. EMBO Mol. Med. 6, 902-917.

Mauch, D.H., Nägler, K., Schumacher, S., Göritz, C., Müller, E.C., Otto, A., and Pfrieger, F.W. (2001). CNS synaptogenesis promoted by glia-derived cholesterol. Science 294, 1354-1357.

Maya Vetencourt, J.F., Sale, A., Viegi, A., Baroncelli, L., De Pasquale, R., O'Leary, O.F., Castrén, E., and Maffei, L. (2008). The antidepressant fluoxetine restores plasticity in the adult visual cortex. Science 320, 385-388.

McGuinn, K.P., and Mahoney, M.G. (2014). Lipid rafts and detergent-resistant membranes in epithelial keratinocytes. Methods Mol. Biol. 1195, 133-144.

Merezhko, M., Pakarinen, E., Uronen, R.-L., and Huttunen, H.J. (2020). Livecell monitoring of protein localization to membrane rafts using protein-fragment complementation. Biosci. Rep. 40, BSR20191290.

Messaoudi, E., Bârdsen, K., Srebro, B., and Bramham, C.R. (1998). Acute intrahippocampal infusion of BDNF induces lasting potentiation of synaptic transmission in the rat dentate gyrus. J. Neurophysiol. 79, 496-499.
Messaoudi, E., Ying, S.-W., Kanhema, T., Croll, S.D., and Bramham, C.R. (2002). Brain-derived neurotrophic factor triggers transcription-dependent, late phase long-term potentiation in vivo. J. Neurosci. 22, 7453-7461.

Meyer-Franke, A., Wilkinson, G.A., Kruttgen, A., Hu, M., Munro, E., Hanson, M.G., Jr., Reichardt, L.F., and Barres, B.A. (1998). Depolarization and cAMP elevation rapidly recruit TrkB to the plasma membrane of CNS neurons. Neuron 21, 681-693.

Minichiello, L. (2009). TrkB signalling pathways in LTP and learning. Nat. Rev. Neurosci. 10, 850-860.

Minichiello, L., Calella, A.M., Medina, D.L., Bonhoeffer, T., Klein, R., and Korte M. (2002). Mechanism of TrkB-mediated hippocampal long-term potentiation. Neuron 36, 121-137.

Monteggia, L.M., Barrot, M., Powell, C.M., Berton, O., Galanis, V., Gemelli, T., Meuth, S., Nagy, A., Greene, R.W., and Nestler, E.J. (2004). Essential role of brain-derived neurotrophic factor in adult hippocampal function. Proc. Natl. Acad. Sci. USA 101, 10827-10832.

Nackenoff, A.G., Moussa-Tooks, A.B., McMeekin, A.M., Veenstra-VanderWeele, J., and Blakely, R.D. (2016). Essential Contributions of Serotonin Transporter Inhibition to the Acute and Chronic Actions of Fluoxetine and Citalopram in the SERT Met172 Mouse. Neuropsychopharmacology 41, 1733-1741.

Nikoletopoulou, V., Lickert, H., Frade, J.M., Rencurel, C., Giallonardo, P. Zhang, L., Bibel, M., and Barde, Y.-A. (2010). Neurotrophin receptors TrkA and TrkC cause neuronal death whereas TrkB does not. Nature 467, 59-63.

Normann, C., Frase, S., Haug, V., von Wolff, G., Clark, K., Münzer, P., Dorner A., Scholliers, J., Horn, M., Vo Van, T., et al. (2018). Antidepressants Rescue Stress-Induced Disruption of Synaptic Plasticity via Serotonin Transporter-Independent Inhibition of L-Type Calcium Channels. Biol. Psychiatry 84, 55-64. Nosé, S. (1984). A molecular dynamics method for simulations in the canonical ensemble. Mol. Physics. 52, 255-268.

Panja, D., and Bramham, C.R. (2014). BDNF mechanisms in late LTP formation: A synthesis and breakdown. Neuropharmacology 76, 664-676.

Park, H., and Poo, M.-M. (2013). Neurotrophin regulation of neural circuit development and function. Nat. Rev. Neurosci. 14, 7-23.

Parrinello, M., and Rahman, A. (1981). Polymorphic transitions in single crystals: a new molecular dynamics method. J. Appl. Physiol. 52, 7182-7190.

Patterson, S.L., Pittenger, C., Morozov, A., Martin, K.C., Scanlin, H., Drake, C., and Kandel, E.R. (2001). Some forms of cAMP-mediated long-lasting potentiation are associated with release of BDNF and nuclear translocation of phospho-MAP kinase. Neuron 32, 123-140.

Pereira, D.B., and Chao, M.V. (2007). The tyrosine kinase Fyn determines the localization of TrkB receptors in lipid rafts. J. Neurosci. 27, 4859-4869.

Pfrieger, F.W., and Ungerer, N. (2011). Cholesterol metabolism in neurons and astrocytes. Prog. Lipid Res. 50, 357-371.

Popova, D., Castrén, E., and Taira, T. (2017). Chronic fluoxetine administration enhances synaptic plasticity and increases functional dynamics in hippocampal CA3-CA1 synapses. Neuropharmacology 126, 250-256.

Pralle, A., Keller, P., Florin, E.L., Simons, K., and Hörber, J.K. (2000). Sphingolipid-cholesterol rafts diffuse as small entities in the plasma membrane of mammalian cells. J. Cell Biol. 148, 997-1008.

Raggi, M.A., Mandrioli, R., Casamenti, G., Bugamelli, F., and Volterra, V. (1998). Determination of fluoxetine and norfluoxetine in human plasma by high-pressure liquid chromatography with fluorescence detection. J. Pharm. Biomed. Anal. 18, 193-199.

Rantamäki, T., Hendolin, P., Kankaanpää, A., Mijatovic, J., Piepponen, P., Domenici, E., Chao, M.V., Männistö, P.T., and Castrén, E. (2007). Pharmacologically diverse antidepressants rapidly activate brain-derived neurotrophic factor receptor TrkB and induce phospholipase-Cgamma signaling pathways in mouse brain. Neuropsychopharmacology 32, 2152-2162.

Rantamäki, T., Vesa, L., Antila, H., Di Lieto, A., Tammela, P., Schmitt, A., Lesch, K.-P., Rios, M., and Castrén, E. (2011). Antidepressant drugs transactivate TrkB neurotrophin receptors in the adult rodent brain independently of BDNF and monoamine transporter blockade. PLoS ONE 6, e20567. 
Remy, I., and Michnick, S.W. (2006). A highly sensitive protein-protein interaction assay based on Gaussia luciferase. Nat. Methods 3, 977-979.

Saarelainen, T., Hendolin, P., Lucas, G., Koponen, E., Sairanen, M., MacDonald, E., Agerman, K., Haapasalo, A., Nawa, H., Aloyz, R., et al. (2003). Activation of the TrkB neurotrophin receptor is induced by antidepressant drugs and is required for antidepressant-induced behavioral effects. J. Neurosci. 23, 349-357.

Sahu, M.P., Nikkilä, O., Lågas, S., Kolehmainen, S., and Castrén, E. (2019) Culturing primary neurons from rat hippocampus and cortex. Neuronal Signal. 3, NS20180207.

Sairanen, M., Lucas, G., Ernfors, P., Castrén, M., and Castrén, E. (2005). Brainderived neurotrophic factor and antidepressant drugs have different but coordinated effects on neuronal turnover, proliferation, and survival in the adult dentate gyrus. J. Neurosci. 25, 1089-1094.

Salo, V.T., Belevich, I., Li, S., Karhinen, L., Vihinen, H., Vigouroux, C., Magré, J., Thiele, C., Hölttä-Vuori, M., Jokitalo, E., and Ikonen, E. (2016). Seipin regulates ER-lipid droplet contacts and cargo delivery. EMBO J. 35, 2699-2716.

Santarelli, L., Saxe, M., Gross, C., Surget, A., Battaglia, F., Dulawa, S., Weisstaub, N., Lee, J., Duman, R., Arancio, O., et al. (2003). Requirement of hippocampal neurogenesis for the behavioral effects of antidepressants. Science 301, 805-809.

Saragovi, H.U., Galan, A., and Levin, L.A. (2019). Neuroprotection: Pro-survival and Anti-neurotoxic Mechanisms as Therapeutic Strategies in Neurodegeneration. Front. Cell. Neurosci. 13, 231.

Schneider, J., Patterson, M., and Jimenez, X.F. (2019). Beyond depression: Other uses for tricyclic antidepressants. Cleve. Clin. J. Med. 86, 807-814.

Senes, A., Engel, D.E., and DeGrado, W.F. (2004). Folding of helical membrane proteins: the role of polar, GxxxG-like and proline motifs. Curr. Opin. Struct. Biol. 14, 465-479.

Shirts, M.R., and Chodera, J.D. (2008). Statistically optimal analysis of samples from multiple equilibrium states. J. Chem. Physiol. 129, 124105.

Sinclair, J.K.L., Walker, A.S., Doerner, A.E., and Schepartz, A. (2018). Mechanism of Allosteric Coupling into and through the Plasma Membrane by EGFR. Cell Chem. Biol. 25, 857-870.e7.

Steinzeig, A., Molotkov, D., and Castrén, E. (2017). Chronic imaging through "transparent skull" in mice. PLoS ONE 12, e0181788.

Suzuki, S., Numakawa, T., Shimazu, K., Koshimizu, H., Hara, T., Hatanaka, H., Mei, L., Lu, B., and Kojima, M. (2004). BDNF-induced recruitment of TrkB receptor into neuronal lipid rafts: roles in synaptic modulation. J. Cell Biol. 167, 1205-1215.

Suzuki, S., Kiyosue, K., Hazama, S., Ogura, A., Kashihara, M., Hara, T., Koshimizu, H., and Kojima, M. (2007). Brain-derived neurotrophic factor regulates cholesterol metabolism for synapse development. J. Neurosci. 27, 6417-6427. Tikka, S., Monogioudi, E., Gotsopoulos, A., Soliymani, R., Pezzini, F., Scifo, E., Uusi-Rauva, K., Tyynelä, J., Baumann, M., Jalanko, A., et al. (2016). Proteomic Profiling in the Brain of CLN1 Disease Model Reveals Affected Functional Modules. Neuromolecular Med. 18, 109-133.

Tulodziecka, K., Diaz-Rohrer, B.B., Farley, M.M., Chan, R.B., Di Paolo, G., Levental, K.R., Waxham, M.N., and Levental, I. (2016). Remodeling of the postsynaptic plasma membrane during neural development. Mol. Biol. Cell 27, 3480-3489.
Ulmschneider, J.P., Smith, J.C., White, S.H., and Ulmschneider, M.B. (2011). In silico partitioning and transmembrane insertion of hydrophobic peptides under equilibrium conditions. J. Am. Chem. Soc. 133, 15487-15495.

Ulmschneider, M.B., Ulmschneider, J.P., Schiller, N., Wallace, B.A., von Heijne, G., and White, S.H. (2014). Spontaneous transmembrane helix insertion thermodynamically mimics translocon-guided insertion. Nat. Commun. $5,4863$.

Vanommeslaeghe, K., Raman, E.P., and MacKerell, A.D., Jr. (2012). Automation of the CHARMM General Force Field (CGenFF) II: assignment of bonded parameters and partial atomic charges. J. Chem. Inf. Model. 52, 3155-3168.

Wang, J., Deng, Y., and Roux, B. (2006). Absolute binding free energy calculations using molecular dynamics simulations with restraining potentials. Biophys. J. 91, 2798-2814.

Wang, Y., Chen, C.H., Hu, D., Ulmschneider, M.B., and Ulmschneider, J.P. (2016). Spontaneous formation of structurally diverse membrane channel architectures from a single antimicrobial peptide. Nat. Commun. 7, 13535.

Welsch, M.E., Kaplan, A., Chambers, J.M., Stokes, M.E., Bos, P.H., Zask, A., Zhang, Y., Sanchez-Martin, M., Badgley, M.A., Huang, C.S., et al. (2017). Multivalent Small-Molecule Pan-RAS Inhibitors. Cell 168, 878-889.e29.

Wray, N.H., Schappi, J.M., Singh, H., Senese, N.B., and Rasenick, M.M. (2019). NMDAR-independent, cAMP-dependent antidepressant actions of ketamine. Mol. Psychiatry 24, 1833-1843.

Wu, X., and Castrén, E. (2009). Co-treatment with diazepam prevents the effects of fluoxetine on the proliferation and survival of hippocampal dentate granule cells. Biol. Psychiatry 66, 5-8.

Yatham, M.S., Yatham, K.S., Ravindran, A.V., and Sullivan, F. (2019). Do statins have an effect on depressive symptoms? A systematic review and metaanalysis. J. Affect. Disord. 257, 55-63.

Zanos, P., Moaddel, R., Morris, P.J., Georgiou, P., Fischell, J., Elmer, G.I., Alkondon, M., Yuan, P., Pribut, H.J., Singh, N.S., et al. (2016). NMDAR inhibitionindependent antidepressant actions of ketamine metabolites. Nature 533, $481-486$.

Zanos, P., Moaddel, R., Morris, P.J., Riggs, L.M., Highland, J.N., Georgiou, P., Pereira, E.F.R., Albuquerque, E.X., Thomas, C.J., Zarate, C.A., Jr., and Gould, T.D. (2018). Ketamine and Ketamine Metabolite Pharmacology: Insights into Therapeutic Mechanisms. Pharmacol. Rev. 70, 621-660.

Zarate, C.A., Jr., Singh, J.B., Carlson, P.J., Brutsche, N.E., Ameli, R., Luckenbaugh, D.A., Charney, D.S., and Manji, H.K. (2006). A randomized trial of an Nmethyl-D-aspartate antagonist in treatment-resistant major depression. Arch. Gen. Psychiatry 63, 856-864.

Zheng, J., Shen, W.-H., Lu, T.-J., Zhou, Y., Chen, Q., Wang, Z., Xiang, T., Zhu, Y.-C., Zhang, C., Duan, S., and Xiong, Z.Q. (2008). Clathrin-dependent endocytosis is required for TrkB-dependent Akt-mediated neuronal protection and dendritic growth. J. Biol. Chem. 283, 13280-13288.

Zheng, C.-Y., Petralia, R.S., Wang, Y.-X., and Kachar, B. (2011). Fluorescence Recovery After Photobleaching (FRAP) of Fluorescence Tagged Proteins in Dendritic Spines of Cultured Hippocampal Neurons. J. Vis. Exp. (50), 2568.

Zonta, B., and Minichiello, L. (2013). Synaptic membrane rafts: traffic lights for local neurotrophin signaling? Front. Synaptic Neurosci. 5, 9. 
CellPress

OPEN ACCESS

\section{STAR $\star$ METHODS}

\section{KEY RESOURCES TABLE}

\begin{tabular}{|c|c|c|}
\hline REAGENT or RESOURCE & SOURCE & IDENTIFIER \\
\hline \multicolumn{3}{|l|}{ Antibodies } \\
\hline anti-TRKB & R\&D Systems & Cat\#AF1494; RRID:AB_2155264 \\
\hline anti-PLC-gamma1 & Cell Signaling Technologies & Cat\#5690; RRID:AB_10691383 \\
\hline anti-TRKB-pY515 & Cell Signaling Technologies & Cat\#4619; RRID:AB_10235585 \\
\hline anti-TRKB-pY816 & Cell Signaling Technologies & Cat\#4168; RRID:AB_10620952 \\
\hline anti-phosphoY & AbD Serotec & Cat\#MCA2472B \\
\hline anti-actin & Santa Cruz & Cat\#sc8432; RRID:AB_626630 \\
\hline anti-GFP & Abcam & Cat\#ab290; RRID:AB_303395 \\
\hline anti-GFP & Abcam & Cat\#ab13970; RRID:AB_300798 \\
\hline anti-flotilin2 & Cell Signaling Technologies & Cat\#3436; RRID:AB_2106572 \\
\hline HRP-conjugated anti-Gt IgG & Invitrogen & Cat\#61-1620; RRID:AB_2533922 \\
\hline HRP-conjugated anti-Rb lgG & BioRad & Cat\#170-5046; RRID:AB_11125757 \\
\hline HRP-conjugated streptavidin & Thermo-Fisher & Cat\#21126 \\
\hline anti-FLAG & Santa Cruz & Cat\#sc807-G; RRID:AB_675757 \\
\hline anti-HRP & Rockland Immunochemicals & Cat\#200-3138-0100; RRID:AB_2611678 \\
\hline \multicolumn{3}{|l|}{ Chemicals, peptides, and recombinant proteins } \\
\hline cholesterol & Sigma-Aldrich & Cat\#C8667 \\
\hline beta-cyclodextrin & Sigma-Aldrich & Cat\#C4555 \\
\hline pravastatin & Orion Pharma & N/A \\
\hline fluoxetine & Bosche Scientific & Cat\#H6995 \\
\hline imipramine & Sigma-Aldrich & Cat\#17379 \\
\hline tritiated imipramine & Perlkin-Elmer & Cat\#NET576250UC \\
\hline chlorpromazine & Sigma-Aldrich & Cat\#C8138 \\
\hline isoproterenol & Tocris & Cat\#1747 \\
\hline diphenhydramine & Tocris & Cat\#3072 \\
\hline flupenthixol & Tocris & Cat\#4057 \\
\hline pimozide & Tocris & Cat\#0937 \\
\hline recombinant human BDNF & Peprotech & Cat\#450-02 \\
\hline biotinylated rh-BDNF & Alomone Labs & Cat\#B-250 \\
\hline NGF & Peprotech & Cat\#450-01 \\
\hline 2R,6R-HNK & Todd Gould & R,R-HNK \\
\hline 2S,6S-HNK & Todd Gould & S,S-HNK \\
\hline ketamine & Pfizer & ketalar $(50 \mathrm{mg} / \mathrm{ml})$ \\
\hline S-ketamine & Esa-Korpi & esketamine \\
\hline neurobasal medium & GIBCO & Cat\#21103049 \\
\hline B27 supplement & GIBCO & Cat\#17504044 \\
\hline DMEM & Lonza & Cat\#BE12-614F \\
\hline fetal calf serum & Sigma-Aldrich & Cat\#F9665 \\
\hline Poly-L-lysine & Sigma-Aldrich & Cat\#P4707 \\
\hline \multicolumn{3}{|l|}{ Critical commercial assays } \\
\hline CellTiterGlo & Promega & Cat\#G7571 \\
\hline Duolink In Situ Red Starter Kit Mouse/Goat & Sigma-Aldrich & Cat\#DU092103 \\
\hline EZ-Link NHS-PEG4 Biotinylation Kit & Thermo Scientific & Cat\#21455 \\
\hline
\end{tabular}




\begin{tabular}{|c|c|c|}
\hline Continued & & \\
\hline REAGENT or RESOURCE & SOURCE & IDENTIFIER \\
\hline \multicolumn{3}{|l|}{ Deposited data } \\
\hline $\begin{array}{l}\text { All experimental data used in the present } \\
\text { study are available in FigShare. }\end{array}$ & https://figshare.com & $\begin{array}{l}\text { https://doi.org/10.6084/m9.figshare. } \\
12698012\end{array}$ \\
\hline \multicolumn{3}{|l|}{ Experimental models: cell lines } \\
\hline cell line & HEK293T & HEK293T \\
\hline cell line & NIH 3T3 stably expressing TRKB & MG87.TRKB \\
\hline cell line & NIH 3T3 stably expressing TRKA & MG87.TRKA \\
\hline cell line & $\mathrm{N} 2 \mathrm{~A}$ & $\mathrm{~N} 2 \mathrm{~A}$ \\
\hline \multicolumn{3}{|l|}{ Experimental models: organisms/strains } \\
\hline $\begin{array}{l}\text { mouse: C57BL/6NTac- } \\
\text { Ntrk2em6006(Y433F)Tac }\end{array}$ & This article & TRKB.Y433F mice \\
\hline mouse: C57BL6-RccHsd & Envigo (Harlan) & TRKB.wt mice \\
\hline mouse: B6.129(Cg)-Slc6atm1Kpl/J & Taconic & SERT.ko \\
\hline rat: Sprague-Dawley & Janvier & Sprague-Dawley rats \\
\hline \multicolumn{3}{|l|}{ Recombinant DNA } \\
\hline Plasmid for PCA & Henri Huttunen & GLuc1C-TRKB.wt/GLuc2C-TRKB.wt \\
\hline Plasmid for PCA & Henri Huttunen & $\begin{array}{l}\text { GLuc1C-TRKB.Y433F/GLuc2C- } \\
\text { TRKB.Y433F }\end{array}$ \\
\hline Plasmid for PCA & Henri Huttunen & GLuc1C-TRKB.wt/GLuc2C-FYN \\
\hline Plasmid for PCA & Henri Huttunen & GLuc1C-TRKB.Y433F/GLuc2C-FYN \\
\hline Plasmid to express GFP-tagged TRKB & Genscript & GFP-TRKB.wt \\
\hline $\begin{array}{l}\text { Plasmid to express GFP-tagged TRKB } \\
\text { mutated at } Y 433\end{array}$ & Genscript & GFP-TRKB.Y433F \\
\hline $\begin{array}{l}\text { Plasmid to express GFP-tagged TRKB } \\
\text { mutated at V437 }\end{array}$ & Genscript & GFP-TRKB.V437A \\
\hline $\begin{array}{l}\text { Plasmid to express GFP-tagged TRKB } \\
\text { mutated at } S 440\end{array}$ & Genscript & GFP-TRKB.S440A \\
\hline $\begin{array}{l}\text { Plasmid to express GFP-tagged TRKB } \\
\text { where TM domain is substuted by TRKA.TM } \\
\text { domain }\end{array}$ & Genscript & GFP-TRKB/TRKA.TM \\
\hline $\begin{array}{l}\text { Plasmid to express FLAG-tagged truncated } \\
\text { TRKB (TRKB.T1) }\end{array}$ & Anna-Kaisa Haapasalo & TRKB.T1 \\
\hline $\begin{array}{l}\text { Plasmid to express FLAG-tagged TM } \\
\text { domain of truncated TRKB (TRKB.T1) }\end{array}$ & Anna-Kaisa Haapasalo & TRKB.T1. $\Delta \mathrm{EC}$ \\
\hline \multicolumn{3}{|l|}{ Software and algorithms } \\
\hline $\begin{array}{l}\text { GROMACS (https://linkinghub.elsevier. } \\
\text { com/retrieve/pii/S2352711015000059) }\end{array}$ & $\begin{array}{l}\text { https://github.com/ElsevierSoftwareX/ } \\
\text { SOFTX-D-15-00003 }\end{array}$ & GROMACS \\
\hline Graphpad Prism v6.01 & https://www.graphpad.com/ & Graphpad Prism \\
\hline JASP & https://jasp-stats.org/ & JASP \\
\hline
\end{tabular}

\section{RESOURCE AVAILABILITY}

\section{Lead contact}

Further information and requests for resources and reagents should be directed to Eero Castren (eero.castren@helsinki.fi).

Materials availability

Plasmids and mouse strain generated in this study are available upon a completed Material Transfer Agreement.

Data and code availability

Experimental data generated in the present study is available in FigShare (https://doi.org/10.6084/m9.figshare.12698012). This study did not generate new code. 


\section{EXPERIMENTAL MODEL AND SUBJECT DETAILS}

\section{Cell culture}

The cell line NIH 3T3 (mouse fibroblasts) stably transfected to express TRKB or TRKA (MG87.TRKB or MG87.TRKA, respectively) were used for in vitro assays (Antila et al., 2014), N2A and HEK293T cells were used to overexpress GFP-tagged TRKB for binding assays. The cells were maintained at $5 \% \mathrm{CO} 2,37^{\circ} \mathrm{C}$ in Dulbecco's Modified Eagle's Medium (DMEM, containing $10 \%$ fetal calf serum, $1 \%$ penicillin/streptomycin, 1\% L-glutamine, and, in the case of MG87.TRKB cells, with $400 \mathrm{mg} / \mathrm{ml}$ of G418). The cell lines used in the present study were not authenticated or genotyped for sex.

Cortex or hippocampus of E18 rat or mouse embryos were dissected and the cells plated in poly-L-lysine coated wells at a $0.5 \times 10^{6}$ cells $/ \mathrm{ml}$ density at $5 \% \mathrm{CO} 2,37^{\circ} \mathrm{C}$ in Neurobasal medium as described in detail (Sahu et al., 2019). The cells were left undisturbed, except for medium change, for 8-22 days depending on the experimental procedure. The primary cultures were not genotyped for sex.

GFP-tagged TRKB.wt or mutants were expressed in cortical or hippocampal cells, HEK293T or in MG87.TRKB cells using Lipofectamine 2000 as transfection agent. All compounds used in pharmacological treatments were diluted in DMSO for in vitro experiments, except NGF and BDNF, diluted in PBS. Cholesterol was dissolved in DMSO and sonicated.

\section{Animals}

C57BL/6NTac-Ntrk2em6006(Y433F)Tac (TRKB.Y433F) mice were generated by introducing the Y433F point mutation into exon 12 of the Ntrk2 gene using CRISPR/Cas9-mediated gene editing with a specific gRNA (Non-Seed_Seed-PAM: TCCTCAGG_ TCTATGCCGTGG-TGG) and HDR (homology directed repair) oligonucleotide (GCAAGGTCATCAGACCTGGCTCTITCTCTCTCCT CAGGTCTTCGCCGTGGTGGTGATTGCATCTGTGGTGGGATTCTGCCTGC). Introduction of the point mutation generates a restriction site for the Bbsl restriction enzyme. The targeting strategy was based on NCBI transcript NM_001025074.2.

\section{Pronucleus injections}

After the administration of hormones, superovulated C57BL/6NTac females were mated with C57BL/6NTac males. One cell stage fertilized embryos were isolated from the oviducts at dpc 0.5 . For microinjection, the one cell stage embryos were placed in a drop of M2 medium under mineral oil. A microinjection pipette with an approximate internal diameter of $0.5 \mu \mathrm{m}$ (at tip) was used to inject the mixed nucleotide preparation (tracrRNA, crRNA, HDR oligo and Cas9 protein) into the pronucleus of each embryo. After recovery, 25-35 injected one cell stage embryos were transferred to one of the oviducts of $0.5 \mathrm{dpc}$, pseudopregnant NMRI females.

\section{Founder analysis}

The genomic DNA was extracted from biopsies and analyzed by PCR. The following templates were used as controls: $\mathrm{H} 2 \mathrm{O}$ (ctrl1), wild-type genomic DNA (ctrl2). A two primer PCR (forward primer: GTTGAGGTTAAAGTGACCTGCTG; reverse primer: TCCTGCTGAGTTAGTCCACACTC) detects the CRISPR/Cas9-induced constitutive knock-in allele as well as potential indel modifications and the unmodified wild-type allele. To distinguish indel modifications from unmodified wild-type sequences, heteroduplex analysis (e.g., via capillary electrophoresis) has to be performed. The PCR amplicons were analyzed using a Caliper LabChip GX device. An aliquot of the PCR reaction was used to validate the presence or absence of the restriction site introduced via homology directed repair. $10 \mathrm{U}$ of $\mathrm{Bbsl}$ enzyme was pipetted into the PCR reaction aliquot and incubated at the appropriate temperature. Bbsl digest results in cleavage of the 493bp PCR product (HDR) in two fragments (362bp and 131bp).

The PCR product of the positive animals from the restriction analysis was subcloned for further characterization of the founder animals. 12 subclones have been prescreened for the integration of the HDR oligonucleotide by restriction with Bbsl. The ratio between the total number of analyzed clones and a positive restriction result allows an estimation of the HDR mosaicism of the founder animals. Subsequently, up to 4 clones were sequenced to confirm correct integration of the HDR oligo and presence of the Y433F point mutation. Finally, the TRKB.Y433F mice were crossed with C57BL6-RccHsd (Envigo-Harlan, the Netherlands), in the University of Helsinki. All animals used from this strain were heterozygous for the Y433F mutation (TRKB.Y433F) or wild-type littermates, and genotyped after weaning.

The SERT-KO mice [line B6.129(Cg)-Slc6atm1Kpl/J] on a C57BL/6 background were purchased from Taconic (Hudson, NY, USA) and bred at the Biomedical Center, University of Freiburg. In every animal, tail biopsies were genotyped with PCR using primers from the Jackson Laboratory database to confirm homozygous knock-out. The SERT-KO mice showed bands at 210 bp, whereas the wild-type mice showed bands at $318 \mathrm{bp}$, which confirmed the gene disruption at the SERT locus. Finally, for in vivo electrophysiology, male adult Sprague-Dawley rats were used.

The present study used males and female mice (18-22 weeks old at the beginning of the experiments), and male Sprague-Dawley rats (15 weeks old at the beginning of the experiments). All animals used in the present study were experimentally naive before the beginning of the described experimental procedures. The animals were group housed (3-6 per cage - type Il: $552 \mathrm{~cm} 2$ floor area - for mice; and 3 per cage - type IV: $1500 \mathrm{~cm} 2$ - for rats, all from Tecniplast, Italy) and randomly allocated into the experimental groups, except when the drug administration was performed in the drinking water. All behavioral experiments were conducted in at least two separated cohorts and analyzed automatically or by experienced observers blind to the treatment/genotype conditions. The number of animals in each experiment was based on previous studies from the literature cited in the correspondent section. For in vivo 
experiments, fluoxetine and pravastatin were dissolved in the drinking water $(0.08$ or $0.1 \mathrm{mg} / \mathrm{l}$ for fluoxetine and $0.08 \mathrm{mg} / \mathrm{l}$ for $\mathrm{pravas}$ tatin) and the volume consumed per cage was monitored every 3 days. R, R-HNK and ketamine were diluted in sterile saline for intraperitoneal injections.

All experimental protocols were approved by the local ethics committees in Finland (\#ESAVI/10300/04.10.07/2016), Norway (\#6159) and Germany (\#G-18-88).

\section{METHOD DETAILS}

\section{In silico methods}

Simulated systems and force fields

The protein simulated in this work included the transmembrane (TM) domains of a TRKB dimer (residues 427-459). For comparison, we also studied a TRKA dimer (residues 410-443). The lipid membranes considered were two-component bilayers composed of varying concentrations of palmitoyl-oleoyl-phosphatidylcholine (POPC) and cholesterol (CHOL). Drug binding studies in the case of TRKB were performed for fluoxetine (FLX). The effects of drugs on the physical properties of lipid membranes were studied for FLX, ketamine, and R,R-HNK. The Charmm36/m force field was used for proteins (Huang et al., 2017) and lipids (Klauda et al., 2010), the TIP3P model was used for water (Jorgensen et al., 1983), and a compatible parameter set was employed for ions (Beglov and Roux, 1994). Parameters for the drugs were generated using the CHARMM general force field (CGenFF) program (Vanommeslaeghe et al., 2012). For details, see below. Table S1 summarizes all simulations and relevant parameters.

Construction of the dimeric protein models

The 3D structure for a segment of mouse TRKB containing its TM domain (residues 427-459) in its monomeric form was generated using the FMAP (Folding of Membrane-Associated Peptides) server (Lomize et al., 2018). The server predicted that the residues V432-A456 form an $\alpha$-helical TM segment.

RosettaMP docking framework (Alford et al., 2015) was used to predict the best dimer configurations to be used in atomistic molecular dynamics (MD) simulations. The orientation of the monomeric helix in a lipid membrane was determined with the OPM server (Lomize et al., 2006). To sample possible TM dimer interfaces and configurations extensively, each helix was rotated around the membrane normal at $30^{\circ}$ intervals and simultaneously also tilted with respect to the membrane normal at $10^{\circ}$ intervals covering a range between $-30^{\circ}$ and $30^{\circ}$, starting from the OPM predicted tilt angle. This procedure resulted in $2 \times 6 \times 12=144$ starting configurations for docking. Rosetta MPdock tool (Alford et al., 2015) was used to generate 960 dimers for each of the 144 starting configurations, yielding a total of 138,240 TM dimer models. These models were sorted by their interface score, and the leading $10 \%$ were clustered into five groups using the Calibur clustering application ( $\mathrm{Li}$ and $\mathrm{Ng}, 2010$ ) based on the models' structural similarity. The structures with the best interface score in each cluster were selected as representative models.

The initial model of the human TRKA TM (amino acids 410-443) dimer was adapted from the NMR structure (PDB:2N90). The initial position of the TRKA TM region in the lipid bilayers was determined using the PPM Server (Lomize et al., 2017).

Exploratory atomistic MD simulations for TRKB model selection and refinement

We initially performed an extensive set of exploratory simulations ( 10 repeats $\times 500 \mathrm{~ns} \times 5$ dimers $\times 2$ membrane compositions) in a membrane environment (POPC: $\mathrm{CHOL}=100: 0$ and POPC: $\mathrm{CHOL}=60: 40$ ) at $363 \mathrm{~K}$ to assess the stability of the selected TRKB TM dimers, and to obtain relaxed structures. The temperature was chosen based on an objective to foster sampling and to find the thermally most stable dimer structures. These exploratory simulations revealed that the only stable dimer model is centrally-coupled (A439-G443) in a cross-like configuration. The simulations of TRKB presented in this study were all started from conformations sampled from the simulations of these dimers. All membrane-protein systems considered in this study were constructed using CHARMM-GUI (Jo et al., 2017).

Drug docking

The drug-binding pocket in the TRKB TM helix dimer was characterized by locally docking FLX to the TRKB TM dimers using RosettaScripts (Fleishman et al., 2011). 30 different cross-like dimer conformations generated by 30 independent 1- $\mu$ s-long MD simulations in a membrane with 20 mol\% cholesterol were used as the "receptor" conformations. FLX was separately placed in the vicinity of the four crevices formed at the dimer interface. The decoys from all docking runs ( 30 conformations $\times 4$ crevices $\times 10,000$ models) were combined, sorted based on interface score, and visually inspected. The best scoring model, where FLX is bound to the crevice facing the extracellular site in a wide-open cross-like dimer conformation, was selected as the basis for further simulations.

Atomistic MD simulations for further exploration and refinement of the drug-binding site

One hundred and twenty independent MD simulations (up to 500 ns each, $\mathrm{POPC}: \mathrm{CHOL}=80: 20,310 \mathrm{~K}$ ) were initiated by randomly repositioning and reorienting the drug in the vicinity of the aforementioned TRKB binding pocket by perturbing it from its docked mode. The effect of net positive charge on the stability of FLX binding was assessed by protonation of FLX in place (6 repeats $\times 500 \mathrm{~ns})$.

\section{Production simulations}

The details of the simulation systems reported in this study are given in Table S1. The preparation of simulation systems and the simulation protocols follows the strategies described above. Systems 1-4 were simulated at $363 \mathrm{~K}$ (Table S1) to improve the sampling of the conformational space of the TRKB dimer structure. In these high temperature simulations, the sampling space was confined to dimeric configurations using half-harmonic flat-bottomed restraints. These studies were guided by recent work on proteins in a membrane environment, 
which has shown simulations at elevated temperature to accurately reproduce the native-state structural equilibria (Ulmschneider et al., 2011, 2014; Wang et al., 2016). The results of the present work are consistent with this conclusion: the protein fold was not disrupted in any of the cases we simulated, and the wild-type TRKB dimers were stable. However, since TRKB dimers including the Y433F mutation were occasionally less stable at the elevated temperature, the half-harmonic flat-bottomed restraint was used to keep the Y433F dimer stable (system 4). Further, the validity of observations for systems 1-4 (Figure 1) were confirmed by additional simulations at $310 \mathrm{~K}$ (systems 5-8). The key results and conclusions were consistent: the simulations at $310 \mathrm{~K}$ revealed the same overall distributions as those in Figure 1 but indicated that each one of those is comprised of 2-3 peaks that are separated by a free energy barrier.

As to the Y433F mutation, the results discussed in this paper refer to the heterozygous variant. Additional simulations for the homozygous variant (not discussed here) revealed similar behavior.

Systems 9-14 (Table S1) were prepared starting from the stable FLX-bound state characterized in exploratory simulations described above. The binding site mutations were introduced in systems 12-14. Drug residence times were calculated for systems $10,12,13,14$.

Simulations of the TRKA TM region (Table S1, systems 15-20) were carried out in the same way as for TRKB.

The estimation of the binding affinity of drugs

For FLX, three independent sets of free energy calculations were performed to estimate the free energy of transfer from the gas phase to the aqueous phase to the membrane phase, and to the protein-binding site at $310 \mathrm{~K}$. All calculations employed the free energy perturbation (FEP) method with Hamiltonian replica exchange (Jiang and Roux, 2010) (exchange attempts at every 1 ps between neighboring windows with an even-odd alternating pattern). Soft-core potentials (Beutler et al., 1994) were employed for both Lennard-Jones and electrostatic interactions ( $\alpha$, the power for lambda term, the power of the radial term, and sigma were set to $0.5,1,6$, and 0.3 , respectively). Each free energy calculation employed 24 windows. For the membrane phase and the protein-binding scenario, the length of the simulations per window was 30-50 ns, and for the aqueous phase $20 \mathrm{~ns}$.

Positional and orientational restraints in the harmonic form were employed to improve phase space overlap in the case of proteinbinding calculations, except for the fully coupled state (Wang et al., 2006). Half harmonic restraints were similarly employed to keep the decoupled molecules within the membrane slab in the membrane-transfer simulations. The lambda values, as well as the strength and the center of the restraints, were adjusted to improve the phase space overlap and exchange rates between the lambda windows.

The free energies and their statistical errors were estimated with the Multistate Bennett Acceptance Ratio (BAR) method (Shirts and Chodera, 2008) using Alchemical Analysis and the pymbar software (Klimovich et al., 2015). A separate set of calculations in the gas phase was performed to estimate the necessary corrections for decoupling the intracellular interactions. The necessary corrections arising from the added restraints and the decoupled intra-molecular interactions were applied to obtain the final free energy values. The effects of the drugs on the physical properties of lipid membranes

To test the possibility that drug-induced modulation of lipid bilayer properties could indirectly activate or contribute to the activation of TRKB, we performed additional protein-free simulations of POPC-cholesterol bilayers with varying $\mathrm{CHOL}$ concentration $(0,20$, and $40 \mathrm{~mol} \%$ ) (Systems 21-50). We used drug-to-lipid ratios of 1:20, 1:10, and 1:5.

Simulation protocols

All simulations were carried out using GROMACS 2018 (Abraham et al., 2015). The equations of motion were integrated using the leap-frog algorithm with a 2 fs time step. All bonds involving hydrogens were constrained using the LINCS algorithm (Hess et al., 1997). Long-range electrostatic interactions were treated by the smooth particle mesh Ewald scheme (Essmann et al., 1995) with a real-space cutoff of $1.2 \mathrm{~nm}$, a Fourier spacing of $0.12 \mathrm{~nm}$, and a fourth-order interpolation. A Lennard-Jones potential with a force-switch between 1.0 and $1.2 \mathrm{~nm}$ was used for van der Waals interactions.

Before the production runs, all protein-membrane systems were equilibrated in stages with the protein heavy atoms kept restrained, at $310 \mathrm{~K}$ and $1 \mathrm{~atm}$ pressure using the Berendsen thermostat and barostat (Berendsen et al., 1984).

All production simulations (Table S1) were performed in the NpT ensemble except for systems 1-4 and 15-17, which were performed in the NVT ensemble with a half-harmonic flat-bottomed restraint in case of TRKB (force constant $1000 \mathrm{~kJ} / \mathrm{mol} / \mathrm{nm} 2$ ) to maintain the inter-helical distance between the G443 $\mathrm{C} \alpha$ atoms below $0.45 \mathrm{~nm}$. The temperature was maintained either at $310 \mathrm{~K}$ or $363 \mathrm{~K}$ (Table S1) using the Nosé-Hoover thermostat (Hoover, 1985; Nosé, 1984). The protein, the membrane, and the solvent (water and $0.15 \mathrm{M} \mathrm{KCl}$ ) were each coupled to separate heat baths at the temperatures indicated in Table S1 with a time constant of $1.0 \mathrm{ps}$. The free energy simulations, on the other hand, employed the leap-frog stochastic dynamics integrator maintaining the temperature at $310 \mathrm{~K}$ with the inverse friction constant for each group set to $2.0 \mathrm{ps}$. Pressure was controlled semi-isotropically using the ParrinelloRahman barostat (Parrinello and Rahman, 1981) with a reference pressure of $1 \mathrm{~atm}$, a time constant of 5 ps, and compressibility of $4.5 \times 10^{-5} \mathrm{bar}^{-1}$ on the $x y$-plane (membrane plane).

The simulation time for systems discussed in this work (the exploratory, the production and the free energy simulations) was over $295 \mu \mathrm{s}$. Including a large variety of test simulations ( 330 $\mu$ s) the total simulation time adds up to more than $625 \mu \mathrm{s}$.

\section{In vitro methods}

\section{Cell viability}

The cell viability assay was performed using CellTiterGlo (\#G7571; Promega) according to manufacturer's instructions. Briefly, equal amounts of medium and the mixture of regents A and B were added to the E18 cortical cells (DIV8) cultivated in clear bottom 96-well plates and incubated for $40 \mathrm{~min}$. The luminescence was analyzed in a plate reader. 


\section{Immunoassays}

The TRKB:pY (phosphorylated TRKB), TRKB:PLC- $\gamma 1$, PLC- $\gamma 1$ :pTRKB.Y816 interactions and were determined by ELISA, based on the general method described in literature (Antila et al., 2014; Fred et al., 2019), with minor adjustments. Briefly, white 96-well plates (OptiPlate 96F-HB, Perkin Elmer) were coated with capturing anti-TRKB or PLC- $\gamma 1$ antibody (1:1000) in carbonate buffer (pH = 9.8) overnight $(\mathrm{ON})$ at $4^{\circ} \mathrm{C}$. Following a blocking step with $2 \% B S A$ in TBS-T $(2 \mathrm{~h}, \mathrm{RT})$, samples were incubated $\mathrm{ON}$ at $4^{\circ} \mathrm{C}$. The incubation with antibody against pTRKB.Y816, PLC- $\gamma 1$ or pY $\left(1: 2000, \mathrm{ON}, 4^{\circ} \mathrm{C}\right)$ was followed by HRP-conjugated anti-Rb lgG (1:5000, $\left.2 \mathrm{~h}, \mathrm{RT}\right)$ or HRP-conjugated streptavidin $(1: 10000,2 \mathrm{~h}, \mathrm{RT})$. Finally, the chemiluminescent signal generated by the reaction with ECL was analyzed in a plate reader (Varioskan Flash, Thermo Scientific).

The surface levels of TRKB were also determined by ELISA (Zheng et al., 2008). Briefly, the MG87.TRKB cells or primary cortical cells from rat or mouse embryos, cultivated in clear bottom 96-well plates (ViewPlate 96, Perkin Elmer), were washed with ice-cold PBS and fixed with $100 \mu$ l of $4 \%$ PFA per well. After washing with PBS and blocking with PBS containing $5 \%$ nonfat dry milk and $5 \%$ BSA, the samples were incubated with primary anti-TRKB antibody (R\&D Systems, \#AF1494, 1:1000 in blocking buffer) or anti-GluR1 subunit of AMPA receptors (CST, \#8850, 1:2000 in blocking buffer) ON at $4^{\circ} \mathrm{C}$. Following washing, the samples were incubated with HRP-conjugated anti-goat or anti-rabbit IgG (1:5000 in blocking buffer) for $1 \mathrm{~h}$ at RT. The cells were washed $4 \mathrm{x}$ with $200 \mu \mathrm{l}$ of PBS for 10 min each. Finally, the chemiluminescent signal generated by reaction with ECL was analyzed in a plate reader.

Protein-fragment complementation assay (Remy and Michnick, 2006) (PCA) was measured upon the reconstitution of enzymatic activity of a humanized Gaussia princeps luciferase (GLuc) following the direct interaction of the proteins of interest in white 96-well plates. Two complementary fragments of the luciferase reporter protein were fused to the intracellular $\mathrm{C}$ terminus domain of TrkB or mutant TrkB(Y433F) to produce the PCA pairs GLuc1C-TRKB.wt/GLuc2C-TRKB.wt and GLuc1C-TRKB.Y433F/GLuc2CTRKB.Y433F. Alternatively, the GLuc1C-TRKB.wt/GLuc2C-FYN or GLuc1C-TRKB.Y433F/GLuc2C-FYN pairs were used The GLuc2C-FYN construct expresses a lipid-raft enriched fragment of Src-family kinase FYN (Merezhko et al., 2020). The GLuc tag was linked via a GS linker that allows the physiological dynamics of TrkB without interference from the presence of the tag. When two TrkB molecules carrying the complementary GLuc fragments interact, the reporter can refold in its original and active conformation thereby producing bioluminescence in the presence of its substrate native coelenterazine. Neuro2A cells, in $10 \%$ ( $/ \mathrm{v}$ ) poly- $\mathrm{L}-$ Lysine coated 96 wells ( 10,000 cells/well) were transfected with the above-mentioned constructs. Cells were treated $48 \mathrm{~h}$ post-transfection with BDNF (10ng/ml/10min), and luminescence measured as a direct indication of TRKB homodimerization with a plate reader (Varioskan Flash, Thermo Scientific, average of 5 measurements, $0.1 \mathrm{~s}$ each) immediately after the injection of the coelenterazine substrate (Nanolight Technology).

The levels of total and phosphorylated TRKB at Y515 or Y816 in MG87.TRKB cells, challenged with BDNF, as well as the levels of PLCg1 and pTRKB.Y816 were measured by western-blotting (Rantamäki et al., 2007).

For the analysis of TRKB migration to lipid rafts, the samples from transfected N2A cells to express GFP-tagged TRKB.wt or TRKB.Y433F, challenged with BDNF, were processed to isolate detergent-resistant membrane (DRM) fractions in sucrose gradient (McGuinn and Mahoney, 2014). N2A cells were seeded at a density of 2.5 million per plate on $10 \mathrm{~cm}$ plates and transfected after 24 hours with either wild-type GFP-tagged full length TRKB or the GFP-tagged Y433F TRKB mutant. 48 hours after plating, cells were washed with ice cold 1x PBS and scraped in extraction buffer ( $25 \mathrm{mM}$ Tris-HCl pH 8, $150 \mathrm{mM} \mathrm{NaCl}, 5 \mathrm{mM}$ EDTA) with the addition of $0.5 \% \mathrm{v} / \mathrm{v}$ Lubrol (Serva) and a cocktail of protease and phosphatase inhibitors (Sigma). Cellular membranes were mechanically broken by passing the cell suspension through a 23G needle five times. Protein concentration was measured for each sample and equal amounts of proteins were transferred to Eppendorf tubes and mixed with sucrose in the extraction buffer to a final concentration of $72 \%$. The samples were then transferred to the bottom of Beckman $2.2 \mathrm{~mL}$ ultracentrifuge tubes and carefully covered with equal volumes of $35 \%$ sucrose and $5 \%$ sucrose in the extraction buffer.

The samples were centrifuged at $52000 \times \mathrm{g}$ for 18 hours at $+4^{\circ} \mathrm{C}$ with a TLS- 55 rotor in a Beckman Coulter XP Optima ultracentrifuge. Finally, 12 fractions per sample, collected from the top of the tube, were transferred to clean tubes, sonicated for 10 minutes in $0.25 \%$ SDS and prepared for western blotting, where the levels of GFP-tagged TRKB and flotillin-2 were analyzed.

Ligand binding assays

The cell-free assays (binding assays) were performed in white 96-well plates, based on the protocol used for western-blotting or ELISA (Baeza-Raja et al., 2016; Das et al., 2015). The plates were precoated with anti-GFP, anti-FLAG, anti-TRK or anti-TRKB antibody (1:1000) in carbonate buffer ( $\mathrm{pH} 9.8)$, ON at $4^{\circ} \mathrm{C}$. Following blocking with $3 \%$ BSA in PBS buffer ( $2 \mathrm{~h}$ at RT), 120 ug of total protein from each sample (of lysates from HEK293T cells transfected to overexpress GFP-TRKB.wt or GFP-TRKB.Y433F) were added and incubated overnight at $4^{\circ} \mathrm{C}$ under agitation. As controls, lysate from MG87 cells expressing TRKA were compared with cells expressing TRKB, and HEK293T cells expressing GFP-TRKB were compared with non-transfected cells, see Figure S4. The plates were then washed $3 x$ with PBS buffer, and the biotinylated fluoxetine or RR-HNK $(0-100 \mu \mathrm{M})$, or a mixture of biotinylated $(1 \mu \mathrm{M}$ of fluoxetine or $\mathrm{R}, \mathrm{R}-\mathrm{HNK})$ and non-biotinylated compounds $(0-10 \mu \mathrm{M})$, was added for $1 \mathrm{~h}$ at RT. The amino-biotinylation of fluoxetine and RR-HNK was performed using a commercial kit (EZ-Link NHS-PEG4 Biotinylation Kit, \#21455, Thermo Scientific) and the reaction monitored by mass spectrometry (Tikka et al., 2016). The luminescence was determined via HRP-conjugated streptavidin (1:10000, 1h, RT) activity reaction with ECL by a plate reader. The luminescence signal from blank wells (containing all the reagents but the sample lystates, substituted by the blocking buffer) was used as background. The specific signal was then calculated by subtracting the values of blank wells from the values of the samples with matched concentration of the biotinylated ligand. For Figure $2 \mathrm{E}$, the precipitated TRKB.wt or Y433F was incubated with tritiated imipramine $(0-30 \mu \mathrm{M})$ and the radioactive emission of the ligand was determined by 
scintillation (OptiPhase Supermix cocktail: \#1200-439; MicroBeta2, Perkin-Elmer). The scintillation signal from blank wells was used as background, and the specific signal was then calculated by subtracting the values of blank wells from the values of the samples with matched concentration of the radioactive ligand.

Microscale Thermophoresis - MST - experiments were performed using Monolith NT.115 (Blue/Red) instrument (NanoTemper Technologies $\mathrm{GmbH}$, Germany). The cell lysates of HEK293T cells expressing GFP-TRKB were used as a source of fluorescently labeled TRKB. HEK293T cells were transfected with GFP-fused TRKB as described and lysed $24 \mathrm{~h}$ after transfection. To evaluate binding of fluoxetine to TRKB, cell lysates were diluted 1.5x times with MST buffer (10mM Na-phosphate buffer, pH 7.4, $1 \mathrm{mM}$ $\mathrm{MgCl} 2,3 \mathrm{mM} \mathrm{KCl}, 150 \mathrm{mM} \mathrm{NaCl}, 0.05 \%$ Tween-20) to provide optimal level of fluorescence. The lysates of non-transfected HEK293T cells were used to evaluate background fluorescence, which appeared to be undetectable in current MST set up. Titration series of fluoxetine $(0-100 \mu \mathrm{M})$ were incubated with diluted cell lysates. The measurements were done in premium coated capillaries (NanoTemper Technologies $\mathrm{GmbH}, \mathrm{MO}-\mathrm{K025}$ ) using LED source with $470 \mathrm{~nm}$ and $50 \%$ infrared-laser power at $25^{\circ} \mathrm{C}$. Hill model was used to evaluate binding affinity, each data point represents mean $\Delta$ Fnorm values from four independent experiments. The bound fraction of the GFP-TRKB was determined and the fitting was performed using the Hill model method incorporated by the MO Affinity Analysis v2.3 software (NanoTemper Technologies).

In situ proximity ligation assay (PLA)

Proximity ligation assay was done with Duolink In Situ Red Starter Kit Mouse/Goat (\#DUO92103, Sigma-Aldrich), following manufacturer's instructions. Briefly, N2A cells (grown in $13 \mathrm{~mm}$ glass coverslips, in DMEM 10\% FBS) were co-transfected by lipofectamine 2000 (ThermoFisher) to express TRKB and farnesylated EGFP and, $24 \mathrm{~h}$ later, were treated with biotinylated fluoxetine (10 $\mu \mathrm{M} / 15 \mathrm{~min})$. Cells were fixed with PFA $4 \%$ for $15 \mathrm{~min}$, washed, and incubated in Duolink blocking buffer for $1 \mathrm{~h}$ at $37^{\circ} \mathrm{C}$. Then, the coverslips were incubated with streptavidin-conjugated HRP (1:5000 in Duolink antibody diluent) for 45 min at room temperature. After washing twice in Duolink washing buffer $A$, samples were incubated overnight at $4^{\circ} \mathrm{C}$ with a mix of goat anti-TRKB (1:1000, \#AF1494, R\&D System) and mouse anti-HRP (1:1000, \#200-3138-0100, Rockland Immunochemicals) diluted in Duolink antibody diluent. After washing in Duolink washing buffer $A$, samples were incubated for $1 \mathrm{~h}$ at $37^{\circ} \mathrm{C}$ with a mix of minus and plus probes diluted 1:5 in Duolink antibody diluent. Then, ligation of probes with connector oligo was done by washing the samples in washing buffer A, and incubating for 30 min at $37^{\circ} \mathrm{C}$ with ligase $(25 \mathrm{U} / \mathrm{ml})$ diluted in the Duolink ligation buffer. Amplification was done by washing the samples in the washing buffer $\mathrm{A}$, and incubating for $100 \mathrm{~min}$ at $37^{\circ} \mathrm{C}$ with polymerase $(125 \mathrm{U} / \mathrm{ml})$ diluted in the amplification buffer. Coverslips were washed in buffer B, then in buffer B diluted 1:100 in milliQ water, and mounted in Duolink in situ mounting medium with DAPI. All incubation steps were done in a humidified chamber.

Images were acquired in z stacks (at least 15 slices, $1.5 \mu \mathrm{m}$ each) with a Zeiss LSM 710 confocal microscope (objective alpha PlanApochromat 63x/1.46 oil Korr M27, with 3x zoom).

Fluorescence recovery after photobleaching

For the fluorescence recovery after photobleaching (FRAP) experiments, E18 rat embryo hippocampal cells were plated onto glass coverslips coated with poly-L-lysine (Sigma-Aldrich) in 4-well cell culture plates at a density of 200.000 cells/well. The cultures were incubated at $+37^{\circ} \mathrm{C}$ in serum-free Neurobasal medium (supplemented with $2 \%$ B27, $1 \%$ L-glutamine and $1 \%$ ampicillin) for approximately 2 weeks (DIV 14-16), renewing half of the growth media weekly. Then, they were transfected with Lipofectamine 2000 (Invitrogen) in order to overexpress GFP-tagged TRKB constructs (TRKB.WT or TRKB.Y433F). The cultures were incubated with the transfection mix (per well: 50ul neurobasal medium, 1ul Lipofectamine 2000, 1ug plasmid DNA) in serum-free media for 1.5 hours, followed by $3 x$ medium washes and returned to the original growth medium after that. The cells were left at $+37^{\circ} \mathrm{C}$ o/n to overexpress the constructs (Koskinen et al., 2012). Next day, FRAP was performed with a confocal microscope (Zeiss LSM 710, Carl Zeiss AG) adapted for live cell imaging with a chamber set at $+37^{\circ} \mathrm{C}$ and $5 \% \mathrm{CO} 2$ (Zeiss Temp Module system, Carl Zeiss AG). Coverslips with the cultured hippocampal neurons were carefully transferred to $35 \mathrm{~mm}$ Petri dishes with warm HBSS medium without phenol red or serum to avoid sources of background fluorescence. After a 10 minutes adaptation period in the microscope chamber to avoid focus drift caused by temperature fluctuations of the stage and objective, dendritic shafts or spines (spine head diameter $=\sim 1 \mathrm{um}$ ) were localized with a $63 \mathrm{x} / 0.90 \mathrm{NA}$ dipping water objective. Picture format was $512 \times 512$ pixels. The nominal speed to capture images was set to 9 (pixel dwell $3.15 \mu$ seconds, taking approximately $0.5 \mathrm{~s}$ to finish a scan), and the pinhole aperture was set to completely open to obtain a strong fluorescent signal (Koskinen et al., 2012; Zheng et al., 2011). Once a region of interest (ROI) was selected with a circle that contained the whole spine, it was bleached to establish the FRAP baseline. In the prebleaching phase, three frames were taken for the purpose of normalization, setting the first frame as $100 \%$ FRAP start value. The bleaching phase consisted of a very short cycle (1-5 bleach iterations, $\sim 0.01-0.5 \mathrm{~s})$ of bleaching to decrease signal of the ROI to $50 \%$. The postbleaching phase lasted for 2 minutes (with a time resolution of $2 \mathrm{~s} /$ frame), until a plateau was reached and the shape of the recovery curve was clear. Next, fluoxetine $(1 \mu \mathrm{M})$, ketamine $(10 \mu \mathrm{M})$ or BDNF $(20 \mathrm{ng} / \mathrm{ml})$ was added to the medium to reach a concentration of and was incubated for 15 minutes. After FRAP establishing baseline, bleaching was performed in different dendritic shafts or spines, and the following recording lasted again for 2 minutes (time resolution of 2 s/frame). Once added to the medium, the drug was present for the rest of the FRAP recording. Finally, for the FRAP image analysis we used the native Zeiss LSM 710 Zen software. The intensity value of the bleached ROI is normalized to a second neighboring unbleached dendritic ROI of the same area and similar initial intensity in order to compensate for the photobleaching generated in the acquisition of the images. That intensity value is then converted to $\%$ by dividing the intensity values of individual frames by the start value of the first frame (t0, 100\%). The mean values from the different experimental groups were then plotted to generate the recovery curve (González-González et al., 2012; Koskinen et al., 2012). 


\section{Immunostaining}

MG87.TRKB or primary cultured cortical cells were fixed with $4 \%$ paraformaldehyde in PBS, and unspecific binding sites were blocked with a blocking buffer (5\% normal donkey serum, 1\% Bovine serum albumin, 0.1\% gelatin, 0.1\% Triton X-100, 0.05\% Tween-20 in PBS). The MG87.TRKB cells were stained with anti-GFP, while primary cultures were incubated with anti-actin. The fluorescence was obtained following incubation with secondary antibodies. The coverslips were mounted with Dako Fluorescence Mounting Medium (\#S3023). Images were acquired with Zeiss LSM710 confocal microscope (63x oil objective).

For Sholl analysis of cultured hippocampal cells, confocal images were processed using FIJI ImageJ (NIH software), and the number of branches in the $80-\mu \mathrm{m}$ range from the cell soma were counted using FIJI plugin.

Super resolution microscopy (dSTORM/TIRF)

For direct stochastic optical reconstruction microscopy (dSTORM) MG87.TRKB cells were grown on cell view 35mm dishes with glass bottom (Greiner) coated with poly-L-lysine (Sigma). Cells were transfected to overexpress TRKB.wt or TRKB.Y433F, and $24 \mathrm{~h}$ later treated with BDNF $(10 \mathrm{ng} / \mathrm{ml})$, fluoxetine $(10 \mu \mathrm{M})$, cholesterol $(20 \mu \mathrm{M})$ or ketamine $(10 \mu \mathrm{M})$ for 15 minutes and fixed as for regular immunofluorescence imaging. Briefly, cells were fixed 24 hours post transfection with 4\% PFA for 20 minutes, washed three times for 5 min with $1 \times$ PBS and incubated in blocking buffer (1\% BSA, 0.1\% gelatin, $5 \%$ goat serum, $0.1 \%$ Triton X-100 and $0.05 \%$ Tween-20 in PBS; all from Sigma) for 1 hour. The primary antibody against GFP (Abcam, \#ab290) was diluted 1:100 and incubated overnight at $+4^{\circ} \mathrm{C}$, while the secondary antibody Alexa Fluor-conjugated goat-anti-rabbit 647 (Invitrogen) was diluted 1:500 and incubated at room temperature for 1 hour. The dishes were stored in $+4^{\circ} \mathrm{C}$ in $1 x$ PBS no longer than 48 hours before imaging.

During imaging, cells were maintained in blinking buffer ( $10 \%$ glucose, $0.07 \%$ cysteamine, $0.75 \mathrm{mg} / \mathrm{ml}$ glucose oxidase and $0.04 \mathrm{mg} / \mathrm{ml}$ catalase in $0.1 \mathrm{M}$ Tris buffer, $\mathrm{pH}$ 7.8-8; all reagents from Sigma-Aldrich), which was freshly prepared and changed every hour. Imaging was performed in TIRF mode (TIRF angle 89.3 calibrated with ring-TIRF at least twice per sample) to specifically image the fluorophores at the plasma membrane. A GE DeltaVision OMX SR system (GE Healthcare) equipped with an Apo N 60X/1.49 oil objective and a sCMOS camera was used to acquire the images. The $640 \mathrm{~nm}$ diode laser was used at $100 \%$ of power to excite the Alexa 647 fluorophores while the $405 \mathrm{~nm}$ laser was adjusted until approximately $50 \%$ of power in order to maintain the blinking throughout the imaging session. For each cell, 35000 frames were acquired, and the localization map of single fluorophores was reconstructed with SoftWoRx 7.0 software (GE Healthcare). For the localization map, the first 1000 frames approximately were discarded and the reconstruction parameters were maintained identical for all the samples.

Reconstruction parameters: For the localization of all the blinking events in the dataset, the point spread function size factor was set at 1.550 with a local maximum factor of 0.05 . Drift correction was adjusted dividing the frames into 20 groups. Tracking of the fluorophores was performed with a maximum localization precision of $100 \mathrm{~nm}$ without fiducial markers. The final localization map of the fluorophores identified was built with a reconstruction pixel size of $10 \mathrm{~nm}$ and localization precision set at $5 \mathrm{and} 100 \mathrm{~nm}$ respectively for the minimum and the maximum, and a fluorophore persistence threshold set at 3 frames.

The analysis of the reconstructed images was performed in Fiji. Depending on the size of picture, 5 or 10 representative regions of interest (ROIs) of $2 \times 1 \mu \mathrm{m}$ were blindly chosen per cell and used for particle analysis of the TRKB-positive clusters. The images acquired were from three independent experiments (Jacobson et al., 2007; Pralle et al., 2000; Salo et al., 2016).

\section{In vivo methods}

\section{Electrophysiology}

In vivo electrophysiology. Intrahippocampal BDNF infusion was performed as described previously (Messaoudi et al., 1998). The experiments were carried out on 20 adult male Sprague-Dawley rats (15 weeks old, Janvier, France). Rats were housed in the animal facility for 1 -week prior to the start of drug administration. Pravastatin was administered in drinking water at a dose of $10 \mathrm{mg} / \mathrm{kg} / \mathrm{day}$ $(0.08 \mathrm{mg} / \mathrm{l}$ solution) for $15-17$ days calculated on the basis of daily body weight.

For electrophysiological experiments, rats were anesthetized with an intraperitoneal injection of urethane (U2500, Sigma-Aldrich) $1.5 \mathrm{~g} / \mathrm{kg}$ body weight, positioned in a stereotaxic frame. The body temperature was constantly monitored and maintained at $37^{\circ} \mathrm{C}$ with an electric heating pad. Holes were drilled in the skull and a concentric bipolar stimulating electrode (Tip separation 500micrometers; SNEX 100; Rhodes Medical Instruments, Woodland Hills, CA) was positioned in the angular bundle for stimulation of the medial perforant path $(7.9 \mathrm{~mm}$ posterior to bregma, $4.2 \mathrm{~mm}$ lateral to the midline, and $2.5 \mathrm{~mm}$ below the dura.

A Teflon-coated tungsten wire recording electrode (outer diameter of $0.075 \mathrm{~mm}$; A-M Systems \#7960) was glued to the infusion cannula (30 gauge). The electrode was then cut so that it extended $800 \mu \mathrm{m}$ from the end of the cannula. The recording electrode was placed in the dentate hilus (3.9 mm posterior to bregma, $2.2 \mathrm{~mm}$ lateral, and 2.8-3.1 mm below the dura). The recording electrode was slowly lowered into the brain with $0.1 \mathrm{~mm}$ increment while monitoring response waveform evoked at $400 \mu \mathrm{A}$ with biphasic rectangular test pulse of $0.033 \mathrm{~Hz}$ (pulse-width $0.15 \mathrm{~ms}$ ). The tip of the infusion cannula was located in the deep stratum lacunosummoleculare of field CA1, $800 \mu \mathrm{m}$ above the hilar recording site and 300-400 $\mu \mathrm{m}$ above the medial perforant synapse. The infusion cannula was connected via a polyethylene (PE50) tube to a $10 \mu \mathrm{l}$ Hamilton syringe and infusion pump.

Responses were allowed to stabilize for 1 hour at a stimulation intensity that produced a population spike $30 \%$ of maximum. After baseline recording for $20 \mathrm{~min}$, infusion of $2 \mu \mathrm{l}$ of $1 \mathrm{ug} / \mathrm{ul} \mathrm{BDNF}$ over $30 \mathrm{~min}$ at a rate of $0.067 \mu \mathrm{l} / \mathrm{min}$. Evoked responses were recorded for $120 \mathrm{~min}$ post infusion. Recorded field potentials were amplified, filtered $(0.1 \mathrm{~Hz}$ to $10 \mathrm{kHz})$, and digitized $(25 \mathrm{kHz})$. Electrophysiological data was analyzed using Datawave Technologies Software company. Changes in the fEPSP slope were expressed in percent of baseline. After recordings rats were decapitated and dentate gyri were dissected and immediately frozen on dry ice and stored at $-80^{\circ} \mathrm{C}$ until use. 
Ex vivo electrophysiology. Extracellular field potentials (fEPSP) were recorded in acute slices of adult mouse hippocampus (Popova et al., 2017). Adult male and female mice (18-22 weeks old) were deeply anaesthetized with isoflurane, the brains were dissected and immersed in ice-cold dissection solution containing (in mM): $124 \mathrm{NaCl}, 3 \mathrm{KCl}, 1.25 \mathrm{NaH} 2 \mathrm{PO} 4,1 \mathrm{MgSO}$, $26 \mathrm{NaHCO}$, $15 \mathrm{D}$-glucose, 9 $\mathrm{MgSO} 4$ and $0.5 \mathrm{CaCl}$. The cerebellum and anterior part of the brain were removed and horizontal $350 \mu \mathrm{m}$ brain slices of the hippocampus were cut on a vibratome. For recovery, the slices were incubated for $30 \mathrm{~min}$ at $31-32^{\circ} \mathrm{C}$ in artificial cerebrospinal fluid (ACSF) containing (in mM): $124 \mathrm{NaCl}, 3 \mathrm{KCl}, 1.25 \mathrm{NaH} 2 \mathrm{PO} 4,1 \mathrm{MgSO} 4,26 \mathrm{NaHCO}, 15 \mathrm{D}$-glucose, and $2 \mathrm{CaCl} 2$ and bubbled with $5 \%$ CO2/ 95\% O2.

Field excitatory postsynaptic currents (EPSPS) were recorded in an interface chamber using ACSF-filled glass microelectrodes (2$4 \mathrm{M} \Omega$ ) positioned in the stratum radiatum of the CA1 region and amplified an Axopatch $200 \mathrm{~B}$ amplifier. Electric stimulation (100 $\mu$ sec duration) was delivered with a bipolar concentric stimulation electrode placed at the Schaffer collateral. Baseline synaptic responses were evoked every $20 \mathrm{~s}$ with a stimulation intensity that yielded a half-maximum response. After obtaining a 15 min stable baseline theta burst stimulation (TBS: 10 bursts of four pulses at $100 \mathrm{~Hz}$, with an interburst interval of $200 \mathrm{msec}$ ) or tetanic stimulation (200ms pulse interval; 100 pulses; $0.1 \mathrm{~ms}$ pulse duration) was delivered and field potentials were recorded for $45 \mathrm{~min}$.

Input/output (I/O) curves were constructed using gradually increased stimulation intensities of 5, 10, 20,30, 40 and $50 \mathrm{mV}$ until the fEPSP reached plateau or visible population spike was seen.

To examine short term plasticity, we performed paired-pulse-facilitation (PPF) experiments using interpulse intervals (IPIs) of 20, 50, 100, and $200 \mathrm{~ms}$ and stimulation intensity evoking half-maximal fEPSP slopes were used. WinLTP (0.95b or 0.96, https://www. winltp.com) was used for data acquisition.

\section{Ocular dominance plasticity}

The "transparent skull" preparation was executed as described in literature (Steinzeig et al., 2017). Briefly, adult female mice (2022 weeks old) were anesthetized via i.p. injection with a mixture containing: 0.05 mg/kg fentanyl (Hameln, Germany); 5 mg/kg midazolam (Hameln, Germany); 0.5 mg/kg medetomidine (Orion Pharma, Finland); diluted in saline (B Braun, Germany). The animals' eyes were covered with protective-gel (Alcon, UK). The animal's head was shaved and disinfected with ethanol and Betadine. The mouse head was then fixed on the stereotaxic frame and the temperature was maintained at $37^{\circ} \mathrm{C}$. A mixture of lidocaine and adrenaline (Orion Pharma, Finland) was applied locally on the head and the skin was cut with spring scissors. The incision area was restricted $2 \mathrm{~mm}$ above the bregma and caudal edge of intraparietal bone. The scalp was removed and a focused air stream and a cotton swab were used to clean and stop bleeding. The periosteum was gently scratched away from the skull with an eye scalpel and the temporal muscles were pushed aside, in order to expose enough space around the area of interest to attach the metal holder. When the skull was smooth, white and dry, fat was removed from the skull rapidly by passing on it a cotton swab soaked in acetone and the air current was used to avoid its penetration into the bone. Then, with a metal stick, a thin layer of cyanoacrylate glue (Loctite 401, Henkel, Germany) was applied to the surface of the skull, in order to make the skull transparent. After waiting 10 minutes for the glue to dry, a first layer of acryl was applied on the skull surface with a brush. Acryl was used to prolong the life of the transparency and was prepared by stirring acrylic powder (EUBECOS, Germany) with methacrylate liquid (Densply, Germany) until a nailpolish consistency was reached. After 30 minutes, a second layer of acryl was applied and was left to dry overnight. Finally, the animals were injected s.c. with $5 \mathrm{mg} / \mathrm{kg}$ Carprofen (ScanVet, Nord Ireland) for postoperative analgesia and i.p. with a wake-up composed by: $1.2 \mathrm{mg} / \mathrm{kg}$ Naloxone (Orpha-Devel Handels und Vertriebs GmbH, Austria), opioid receptor antagonist; $0.5 \mathrm{mg} / \mathrm{kg}$ Flumazenil (Hameln, Germany), GABA-A receptor antagonist; $2.5 \mathrm{mg} / \mathrm{kg}$ Atipamezole (Vet Medic animal Health Oy, Finland), adrenergic receptor antagonist; diluted in saline (B Braun, Germany). The next day, isoflurane at $4 \%$ was used for a couple of minutes to induce the anesthesia and then it was reduced at $2 \%$ for the procedure. The acryl layer was polished only on the area of interest, using a hand drill with a polishing bit. A metal head holder was first glued on the skull, carefully keeping the area of interest at the center of the holder, and then fixed with a mixture of cyanoacrylate glue and dental cement (Densply, Germany). Finally, transparent nail polished (\#72180, Electron Microscopy Sciences) was applied inside the metal holder above the area of interest.

Monocular deprivation. -Isoflurane at $4 \%$ was used for a couple of minutes to induce the anesthesia and then it was maintained at $2 \%$ until the end of the procedure. A drop of antibiotic eye gel (Isothal Vet 1\%, Dechra, Canada) was applied on the left eye, the eyelashes were cut and the eye was sutured shut with 3 mattress sutures. At the end, antibiotic ointment (Oftan Dexa-Chlora, Anten, Finland) was applied on the sutured eye and Carprofen $(5 \mathrm{mg} / \mathrm{kg})$ was injected s.c. for postoperative analgesia. The monocular deprivation lasted 8 days and during that period, all animals were checked daily and resutured when showed a sign of the thread loss to prevent reopening of the eyes.

Optical imaging. The saturation of haemoglobin in the primary visual cortex of the right hemisphere was measured to assess cortical activity (Cang et al., 2005; Kalatsky and Stryker, 2003). The animals were anesthetized with 1.8\% isoflurane with a 1:2 mixture of $\mathrm{O} 2$ :air for 15 minutes and then maintained at $1.2 \%$ isoflurane for at least 10 minutes before starting the imaging session. Two sessions of imaging were performed: one before the beginning of the treatment administration (IOS I) and one on the 8th day after monocular deprivation (IOS II).

Optical Imaging Apparatus. The animals were kept on a heating pad located in front of and within $25 \mathrm{~cm}$ from the stimulus monitor. The head holder was firmly fixed and the animal's nose was aligned to the midline of the stimulus monitor. The visual stimulus was a $2^{\circ}$ wide horizontal bar moving upward with a temporal frequency of $0.125 \mathrm{~Hz}$ and a spatial frequency of $1 / 80$ degree, displayed in the central part of a high refresh rate monitor ( -15 to 5 degree azimuth, relative to the animal visual field) in order to preferentially stimulate the binocular part of the visual field. First, a green light $(540 \pm 20 \mathrm{~nm})$ was used to illuminate the skull surface in order to find the 
region of interest and acquire a map of the surface vascular pattern, then the camera was focused $600 \mu \mathrm{m}$ below the pial surface and the green light switched with a red light $(625 \pm 10 \mathrm{~nm})$, to record the intrinsic signal. A longpass red filter (590 nm) was put between the skull surface and the camera to avoid that light from the stimulus monitor could affect the intrinsic signal acquisition. When the signal from one eye was being recorded, the other eye was protected with viscotears and covered with a patch. The continuous-periodic stimulation was synchronized with a continuous frame acquisition, frames were collected independently for each eye at a rate of 30 fps for 5 minutes and stored as a $512 \times 512$ pixel image, after spatial binning of the camera images.

Data analysis. An analysis software package designed by Kalatsky et al. was used to perform Fourier decomposition on the cortical maps, that allowed to extract the intrinsic signal from the biological noise (Kalatsky and Stryker, 2003). The intrinsic response was presented as fractional changes in skull surface reflectance $\times 104$ and its magnitude was used to calculate the activation of the visual cortex in the right hemisphere due to ipsilateral or contralateral eye stimulation. A low-pass filter (uniform kernel of $5 \times 5$ pixel) was applied to the ipsilateral magnitude map to smoothen it and then, in the resulting map, the $30 \%$ of the peak response amplitude was set as threshold in order to eliminate background noise and to define the area that produced the strongest response to the ipsilateral eye. This smoothened and thresholded map was used as a mask to select the binocularly responsive region of interest within the visual cortex. After obtaining cortical maps for both contralateral (C) and ipsilateral (I) eyes and computing Ocular Dominance score as $(\mathrm{C}-\mathrm{I}) /(\mathrm{C}+\mathrm{l})$, finally, the Optical Dominance Index (ODI) was calculated as the mean of the OD score for all responsive pixels (Cang et al., 2005; Greifzu et al., 2014). The ODI values are contained in an interval going from -1 to +1 : positive values indicate a contralateral bias, negative ones indicate ipsilateral bias and ODI values of 0 indicate that ipsilateral and contralateral eyes are equally strong (Steinzeig et al., 2017).

\section{Behavioral analysis}

Object-location memory (OLM): this test was performed in a square arena ( $28 \mathrm{~cm}$ side) with opaque walls containing cues (black stripes or spots). The adult male and female mice (18-22 weeks old) were placed for 3 consecutive days (15min per session) in the arena with two identical objects (table tennis balls glued to caps of 50ml Falcon tubes) in the same position throughout the sessions (pretest). At the test session one of the objects is moved to a different position and the number of visits (counted as sniffing or interacting with the object) to the old $(A)$ or newly located $\left(A^{\prime}\right)$ object was determined by an observer bling to the conditions (Ampuero et al., 2013; Dere et al., 2007).

Contextual fear conditioning: this test was modified from previous studies (Karpova et al., 2011). Briefly, adult male and female mice (18-22 weeks old) were conditioned to 5 scrambled foot shocks $(0.6 \mathrm{~mA} / 2 \mathrm{~s})$ during the 8 min session (arena: $23 \times 23 \times$ $35 \mathrm{~cm}$ ) under constant illumination (100 lux). During the extinction trials, the animals were exposed to the same context where the shocks were delivered and the time spent in freezing during the $8 \mathrm{~min}$ session was automatically determined by the software (TSE Systems, Germany).

Forced swimming test: animals (adult male and female mice, 18-22 weeks old) were placed in $5 \mathrm{l}$ glass beaker cylinders (19cm diameter, with $20 \mathrm{~cm}$ water column) for $6 \mathrm{~min}$. The immobility was assessed in the last $4 \mathrm{~min}$ of the session (Diniz et al., 2018). The water was changed between each test. After swimming, animals were towel-dried and kept in a warmed cage before returning to their home cages. Test was videotaped and analyzed by a trained observer blind to treatment.

Incorporation of BrdU

Animals: both males and females were used in this experiment. The Bromodeoxyuridine (BrdU) (Sigma) was administered intraperitoneally at the dose of $75 \mathrm{mg} / \mathrm{kg}$ four times every $2 \mathrm{hr}$ to reach $300 \mathrm{mg} / \mathrm{kg}$ total for each animal. The injection procedure was carried out at the start of the treatment (fluoxetine $15 \mathrm{mg} / \mathrm{kg} /$ day in the drinking water for 3 weeks, solution at $0.1 \mathrm{mg} / \mathrm{l})$. On day $21 \mathrm{of}$ the drug treatment, the animals were euthanized by $\mathrm{CO} 2$, brains were quickly removed, followed by hippocampus dissection on ice, instantly frozen on dry ice and stored at $-80^{\circ} \mathrm{C}$ until further use.

A semiquantitative dot-blot method was performed as previously described with minor modifications (Wu and Castrén, 2009). To isolate the DNA of the hippocampus samples, DNeasy ${ }^{\circledR}$ Blood and Tissue Kit (QIAGEN, Germany) was used. The extraction procedure was as per the manufacturer's instruction. The DNA purity was assessed by the spectrophotometer NanoDrop 2000C (ThermoFisher Scientific, USA). DNA was incubated with 1 volume of $4 \mathrm{~N} \mathrm{NaOH}$ solution for 30 min at room temperature to render it as single stranded and immediately kept on ice to prevent reannealing. The DNA solution was neutralized by an equal volume of $1 \mathrm{M}$ Tris- $\mathrm{HCl}$ ( $\mathrm{pH}$ 6.8). The single-stranded neutralized DNA (1ug) was dot-blotted onto a nylon transfer membrane (Schlleicher and Schuell, Keene, $\mathrm{NH}$ ) with a dot-blot apparatus (Minifold, Schlleicher and Schuell) under vacuum and the DNA was fixed by ultraviolet cross-linker $(1200 \mu \mathrm{J} \times 100$, Stratagene, La Jolla, CA). These membranes were processed as mentioned in the previous publication. The mouse anti BrdU monoclonal antibody (1:1000, B2531, Sigma) was used as the primary antibody and anti-mouse horseradish peroxidase (HRP) (Bio-Rad, USA) as the secondary antibody. The Pierce ECLplus kit (Thermo Fisher scientific, USA) was used as a chemiluminescent method to develop the membrane. The membranes were scanned by imaging using a Fuji LAS-3000 Camera (Tamro Medlabs, Finland) and the densitometry analysis was performed by ImageJ Software.

Quantification of brain fluoxetine levels

The brain samples were homogenized in $200 \mu \mathrm{L}$ of $100 \%$ methanol (LiChrosolv, Merck, Darmstadt, Germany) with an ultrasound sonicator (GM35-400, Rinco Ultrasonic, Switzerland) continuously for at least $30 \mathrm{~s}$. The homogenates were centrifuged at $20800 \mathrm{~g}$ for $35 \mathrm{~min}$ at $4{ }^{\circ} \mathrm{C}$. The supernatant was then removed to $0.5 \mathrm{~mL}$ Vivaspin filter concentrators $(10,000$ MWCO PES; Vivascience AG, Hannover, Germany) and centrifuged at $8600 \times \mathrm{g}$ at $4^{\circ} \mathrm{C}$ for $35 \mathrm{~min}$. 


\section{CellPress}

OPEN ACCESS

The HPLC system for determination of the concentration of fluoxetine consisted of a solvent delivery pump (Jasco model PU-1580 HPLC Pump, Jasco International Co, Japan) connected to an online degasser (Jasco 3-Line Degasser, DG-980-50) and a ternary gradient unit (Jasco LG-1580-02), an analytical column (Kinetex C-18 $5 \mu \mathrm{m}, 4.60 \times 50 \mathrm{~mm}$, Phemomenex Inc) protected by a 0.5$\mathrm{mm}$ inlet filter and thermostated by a column heater (CROCO-CIL, Cluzeau Info-Labo, France), and a fluorescence detector (Jasco Intelligent Fluorescence Detector model FP-1520). The wavelengths of the fluorescence detector were set to 290 (excitation) and 230 (emission), which have been found to be optimized for fluoxetine (Raggi et al., 1998). The mobile phase consisted of 0.1 M NaH2PO4 buffer (Merck, D), pH 2.7 (adjusted with phosphoric acid), 35\% (v/v) acetonitrile (LiChrosolv, Merck, Darmstadt, Germany), and the flow-rate was $1.0 \mathrm{ml} / \mathrm{min}$. Ten microliters of the filtrate was injected onto the column with a refrigerated autoinjector (Shimadzu NexeraX2 SIL-30AC, Shimadzu Corp, Japan). The chromatograms were processed by AZUR chromatography data system software (Cromatek, Essex, UK). The results can be found in Table S2.

\section{QUANTIFICATION AND STATISTICAL ANALYSIS}

The data in the present study was analyzed by Student's t test (two-tailed, paired or independent); one- or two-way ANOVA were also applied, followed by Fisher's LSD post hoc test using GraphPad Prism (https://www.graphpad.com/) v6.01; or multivariate ANOVA using JASP (https://jasp-stats.org/) v.0.14.1. The $F$ and $p$ values are indicated in the figure legends. All experimental data used in the present study are available in FigShare under CC-BY license (https://doi.org/10.6084/m9.figshare.12698012). 


\section{Supplementall Figures}

A

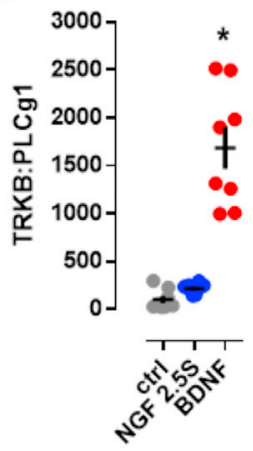

$\mathbf{F}$

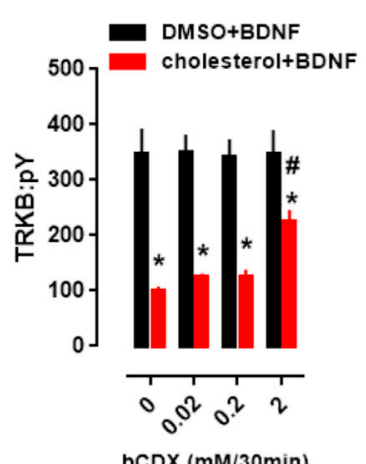

bCDX (mM/30min)

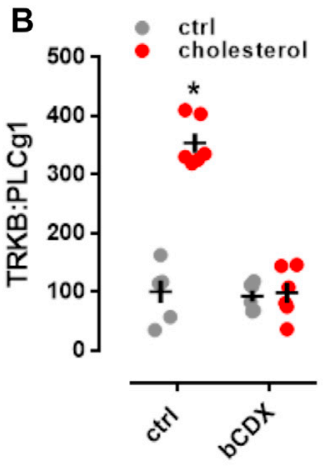

G

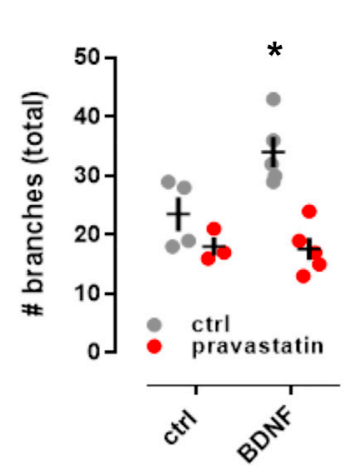

C

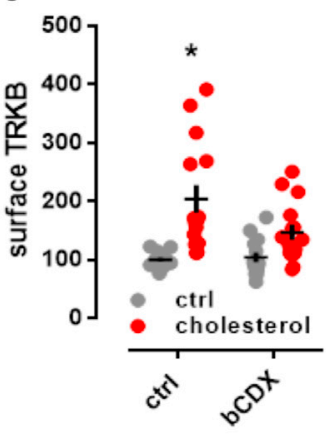

D

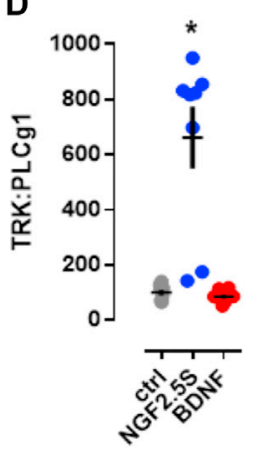

E

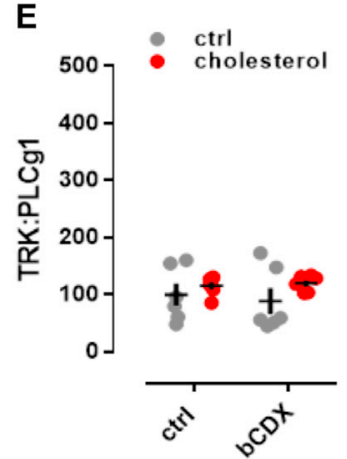

$\mathbf{L}$
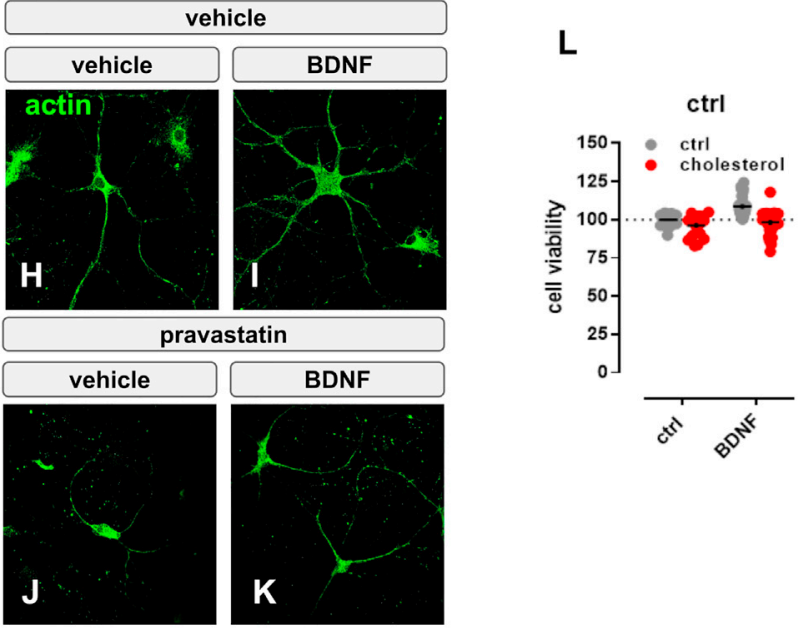

$\mathbf{P}$

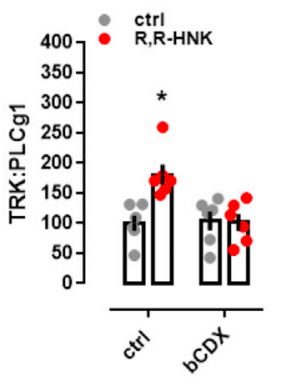

Q

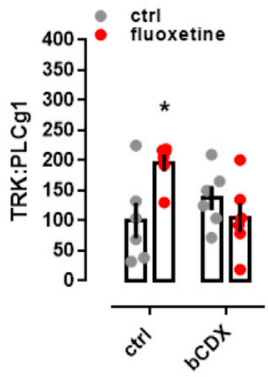

Figure S1. Cholesterol sensing by TRKB, related to Figure 1

(A-E) MG87 cells were treated with $\beta$-cyclodextrin (bCDX), NGF, BDNF, or cholesterol and the levels of TRKB:PLC- $\gamma 1$ or surface TRKB determined by ELISA. In MG87 cells expressing TRKB, (A) BDNF (10ng/ml/15min), but not NGF $(50 \mathrm{ng} / \mathrm{ml} / 15 \mathrm{~min})$, increases the TRKB:PLC- $\gamma 1$ interaction [treatment: $F(2,21)=46.24$; $p=0.0001]$ measured by ELISA. The effect of cholesterol $(20 \mu \mathrm{M} / 15 \mathrm{~min})$ on (B) TRKB:PLC- $\gamma 1$ coupling [interaction: $F(1,20)=59.49 ; p=0.0001]$ and $(C)$ surface positioning of TRKB [interaction: $F(1,54)=4.202 ; p=0.04$ ] is counteracted by pre-treatment with beta-cyclodextrin (bCDX; $2 \mathrm{mM} / 30 \mathrm{~min}$ ). In MG87 cells expressing TRKA, (D) NGF, but not, BDNF, increases the TRKA:PLC- $\gamma 1$ coupling [treatment: $F(2,21)=25.29 ; p=0.0001]$. (E) Lack of effect of cholesterol-induced TRKA:PLC- $\gamma 1$ in cells expressing TRKA [interaction: $F(1,20)=0.25 ; p=0.64$ ]. (F) Rat cortical cells were treated with different concentrations of bCDX (30min), challenged by a combo of cholesterol+BDNF (15min), and the levels of TRKB:pY was determined by ELISA. $\beta$-cyclodextrin ( $\mathrm{mM} / 30 \mathrm{~min})$ reverses the block of BDNF-induced pTRKB $(10 \mathrm{ng} / \mathrm{ml} / 15 \mathrm{~min})$ by high cholesterol concentration $(100 \mu \mathrm{M} / 15 \mathrm{~min})[\mathrm{F}(1,40)=96.95, \mathrm{p}<0.0001, \mathrm{n}=6 / \mathrm{group}]$. (G) Rat hippocampal cells were treated with pravastatin and BDNF, fixed and stained for actin. Effect of pravastatin ( $1 \mu \mathrm{M} / 3$ days) on BDNF-induced neurite branching (10ng/ml/3days); interaction: $F(1,13)=4.967, p=0.0441, n=3-5]$. $(H-K)$ representative images of pravastatin effect on BDNF-induced branching. (L,M) Rat cortical cells were 
treated with pravastatin, cholesterol and BDNF, and the cell viability determined by CellTiterGlo. Pravastatin-induced cell death ( $2 \mu \mathrm{M} / 5$ days $)$ is counteracted by co-incubation with cholesterol $(20 \mu \mathrm{M} / 5$ days) and BDNF (10ng/ml/5days) [interaction: $F(1,164)=10.895, p=0.001, n=20-24$ ]. Data expressed as mean $\pm S E M$ of percentage from ctrl group. ${ }^{*} p<0.05$ from the control group (Fisher's LSD). (N-Q) Rat cortical cells were treated with $\beta$-cyclodextrin (bCDX) or antidepressants, and the levels of TRKB:PLC- $\gamma 1$ determined by ELISA. The pretreatment with bCDX (2mM/30min) prevents the increase in TRKB:PLC- $\gamma 1$ (PLCg1) induced by (N) imipramine [interaction: $F(1,20)=14.71, p=0.0010, n=6$ /group], $(O)$ ketamine [interaction: $F(1,19)=9.335, p=0.0065, n=5-6],(P) R, R-H N K$ [interaction: $F(1,20)=$ 8.033, $p=0.0102, n=6 /$ group] or $(Q)$ fluoxetine [interaction: $F(1,20)=8.035, p=0.0103, n=6 /$ group]. ${ }^{*} p<0.05$ from the control group (Fisher's LSD). 
A

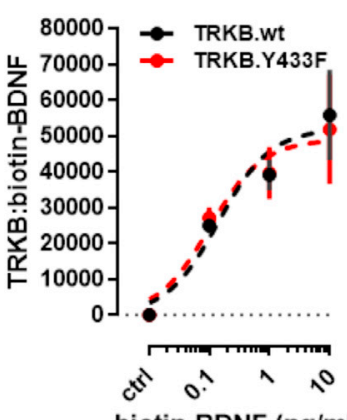

biotin-BDNF (ng/ml)
B

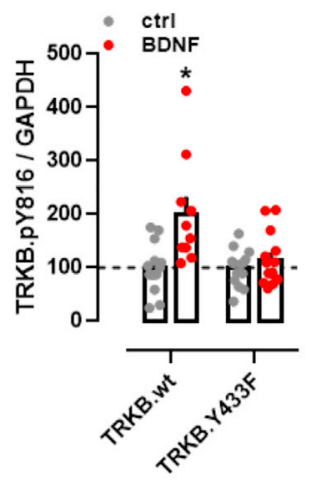

C

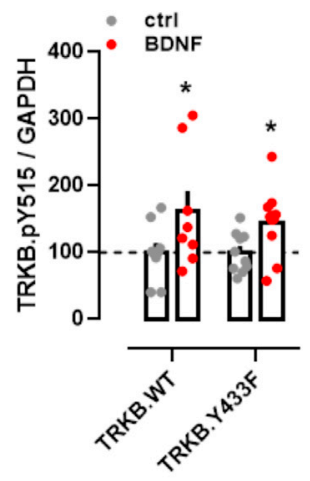

D

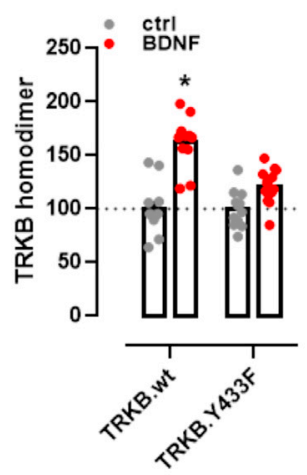

E

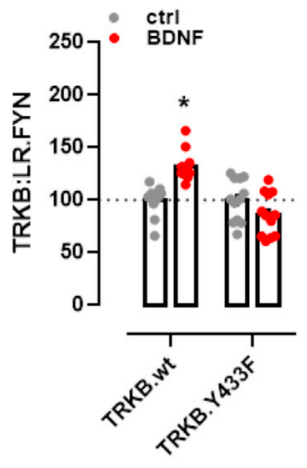

F

ctrl

BDNF
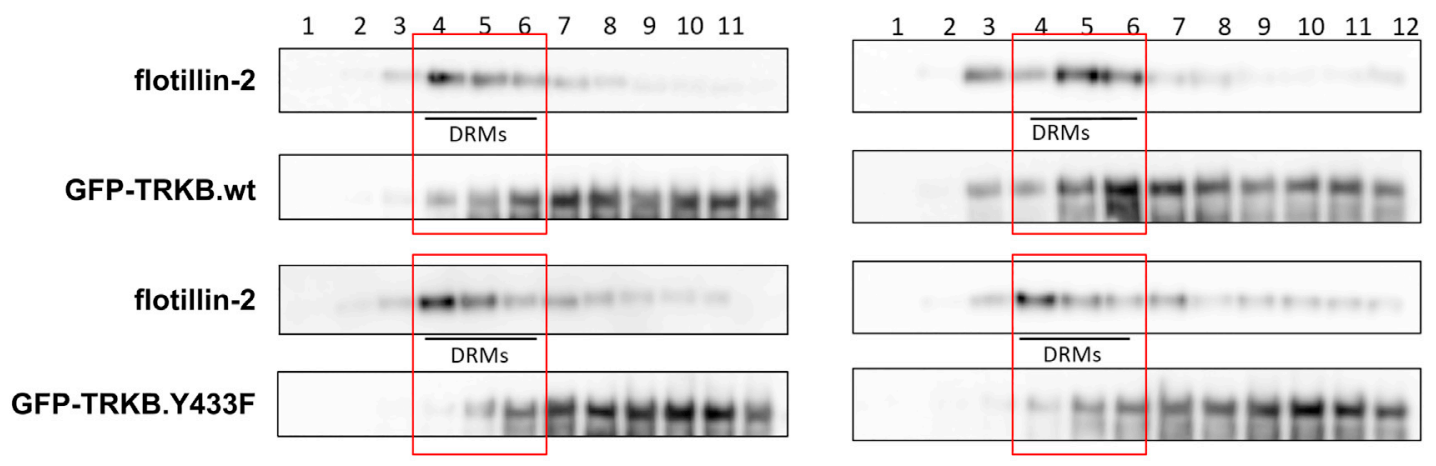

G

IP: PLCg1

\begin{tabular}{|llll|}
\hline 1 & 2 & 3 & 4 \\
\hline & & & \\
\hline
\end{tabular}

PTRKB.Y816

PLCg1
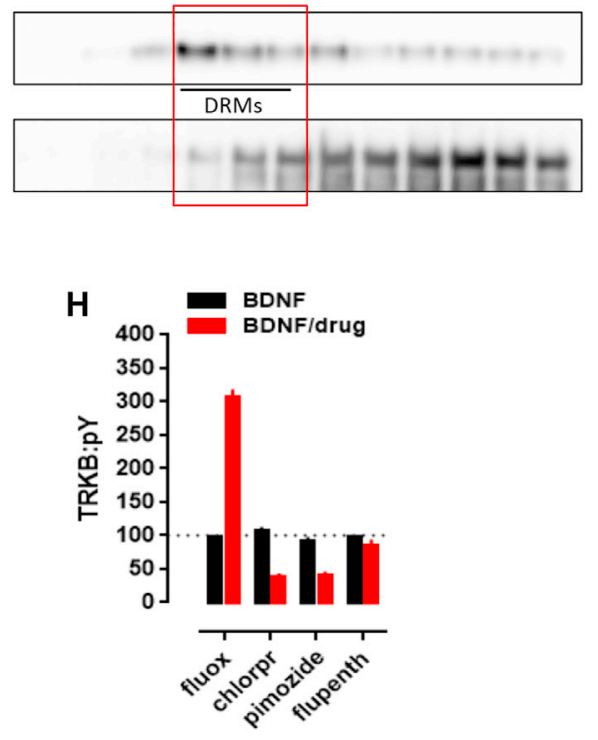

cholesterol 50um

Figure S2. Antidepressants bind to TRKB transmembrane domain, related to Figure 2

(A) Lysates from HEK293T cells transfected to express TRKB were submitted to ligand binding assay. BDNF interaction with TRKB is not altered by the Y433F mutation ( $n=6$ /group). See schematics in S5A. (B,C) MG87 cells transfected to express TRKB were treated with BDNF and the levels of pTRKB determined by western-blotting. BDNF-induced phosphorylation of TRKB at (B) Y816 is prevented in the TRKB. Y433F mutant [interaction: $F(1,47)=6.688, p=0.0129 ; n=10-14]$, but the Y433F mutation does not affect BDNF-induced phosphorylation of TRKB at (C) Y515 residues in MG87 cells [interaction: $F(1,33)=0.1874, p=0.6679$; $n=9-10]$. (D,E) N2A cells transfected to express luciferase-tagged TRKB and/or raft-restricted FYN, were treated with BDNF and submitted to PCA. (D) The BDNF-induced dimerization of TRKB is compromised by the $Y 433 F$ mutation [interaction: $F(1,42)=11.08, p=0.0018 ; n=11$-12]. (E) The BDNF-induced increase in TRKB interaction with FYN fragment in lipid raft is compromised by the $Y 433 F$ mutation [interaction: $F(1,44)=20.96, p<0.000 ; n=12$ ]. Data expressed as mean \pm SEM of percentage from ctrl group. ${ }^{*} p<0.05$ from the control group (Fisher's LSD). (F) N2A cells transfected to express TRKB were treated with BDNF and submitted to fractionation of membrane components. The Y433F mutation prevents BDNF-induced $(10 \mathrm{ng} / \mathrm{ml} / 15 \mathrm{~min})$ translocation of TRKB to lipid-rafts in N2A cells (DRM: detergent-resistant membranes; 1 of 2 replicas). Rat cortical cells were treated with bCDX and fluoxetine, and the levels of surface TRKB determined by ELISA. Rat cortical cells were treated with fluoxetine or ketamine and submitted to immunoprecipitation of PLC- $\gamma 1$ and western-blotting for TRKB and PLC- $\gamma 1$. (G) Representative western-blotting of co-immunoprecipitation of PLC- $\gamma 1$ and TRKB phosphorylated at Y816 in cultured cortical cells of rat embryo (1 of 2 
replicas); lane 1: ctrl, 2: ctrl, 3: fluoxetine $(10 \mu \mathrm{M} / 15 \mathrm{~min})$, 4: ketamine (10 $\mu \mathrm{M} / 15 \mathrm{~min})$. (H) Rat cortical cells were preincubated with cholesterol (50uM) and fluoxetine, chlorpromazine, pimozide or flupenthixol (10uM) for $15 \mathrm{~min}$ and challenged with BDNF (10ng/ml/15min). The levels of TRKB:pY were determined by ELISA [interaction: $F(3,64)=181.9, p<0.0001, n=9$ /group]. Data expressed as mean \pm SEM of percentage from ctrl group. ${ }^{*} p<0.05$ from the control group (Fisher's LSD). ${ }^{*} p<0.05$ from the control group (Fisher's LSD). 
A
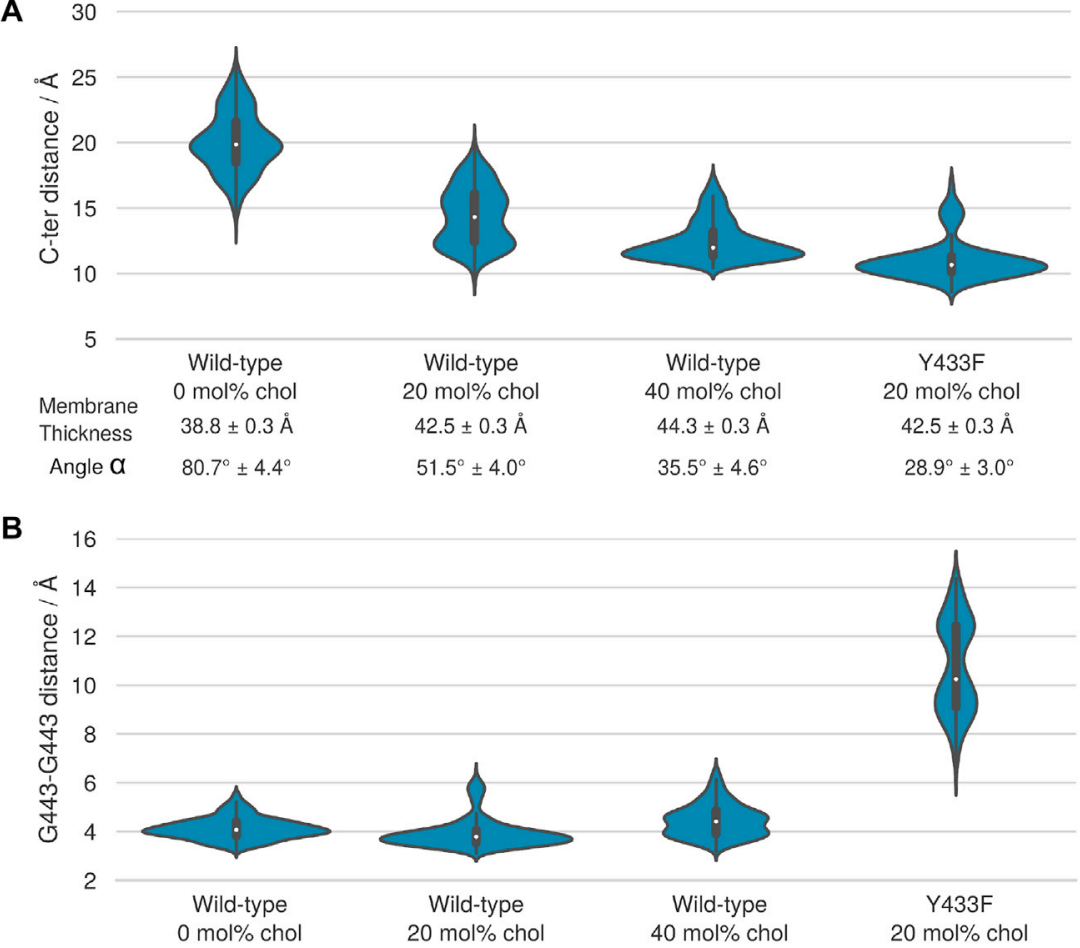

C

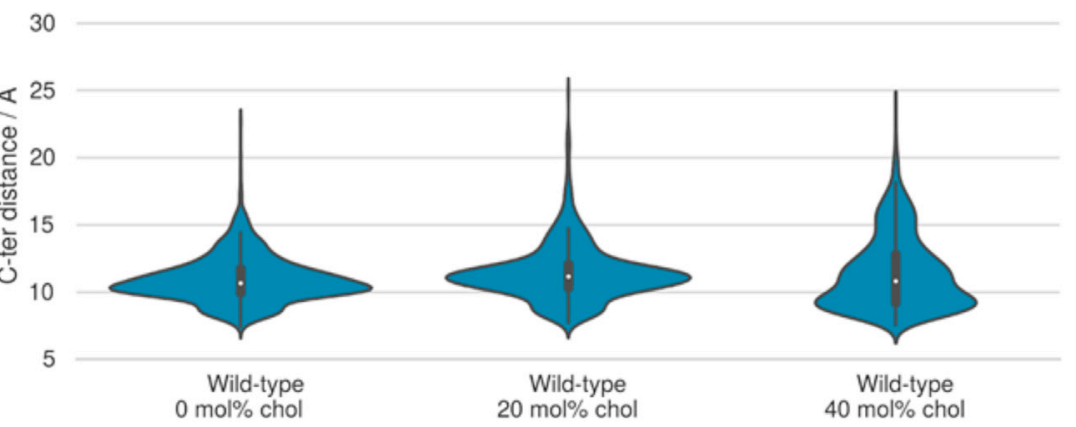

Figure S3. Cholesterol sensing by TRKB, related to Figures 1 and 3

(A) The distribution of the distance between the C-terminal residues of the monomers (center of mass L451-L453 C $\alpha$ atoms (indicated with an arrow in Figure 1) are shown as violin plots. Increasing cholesterol concentration increases membrane thickness, which for the wild-type decreases the C-terminal distance. Y433F results in the disruption of the dimerization interface and the cross-like conformation. The parallel-like conformation of the WT-Y433F dimer appears to have a smaller hydrophobic length than that of the individual WT helices. Given at the bottom are average values for the membrane thickness (phosphate-phosphate distance) and the average angle between the helices $a$. [Kruskal-Wallis: $H=27.8736 ; p<0.001 ; n=10 /$ group]. (B) The effect of cholesterol concentration and the $\mathrm{Y} 433 \mathrm{~F}$ mutation on the stability of the interdimeric interface. The stability of the dimerization interface is characterized by a distribution of the distance between the monomers' $\mathrm{C} \alpha$ carbons of G443 shown as violin plots for wild-type at different cholesterol concentrations and for the Y433F heterozygous mutant at 20 mol\% cholesterol concentration (systems 1-4, Table S1) [Kruskal-Wallis: $\mathrm{H}=25.4385 ; \mathrm{p}<0.001 ; \mathrm{n}=10$ /group]. The results demonstrate that the $\mathrm{Y} 433 \mathrm{~F}$ mutation results in a total disruption of the A439-G443 dimerization interface. (C) The distribution of the distance between the C-terminal residues of the monomers (center of mass L439-L437 C $\alpha$ atoms) in the TRKA transmembrane domain shown as violin plots. The results indicate that cholesterol concentration has no notable effect on the distance between the C-terminal residues of the two monomers in the TRKA TM dimer. In essence, TRKA is non-responsive to changes in cholesterol concentration (systems 12-14, Table S1). 
A

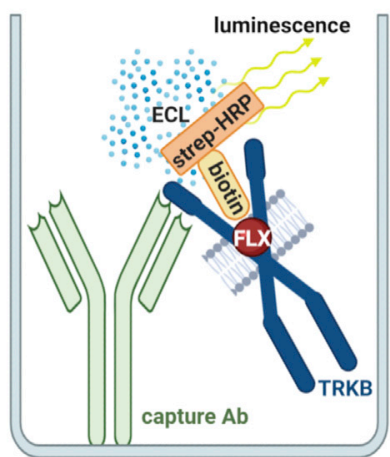

B

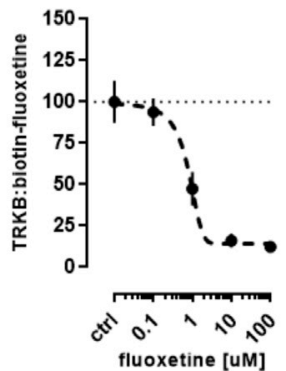

C

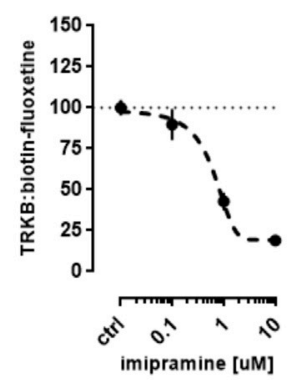

D

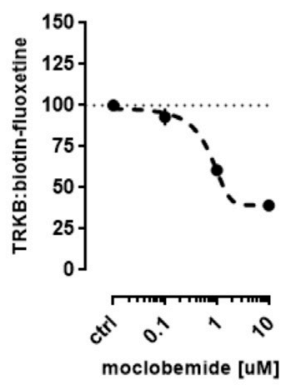

E

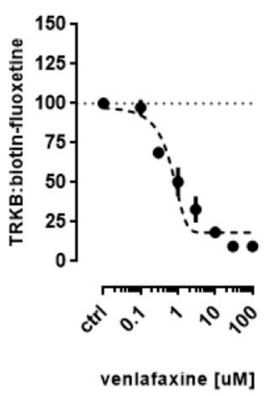

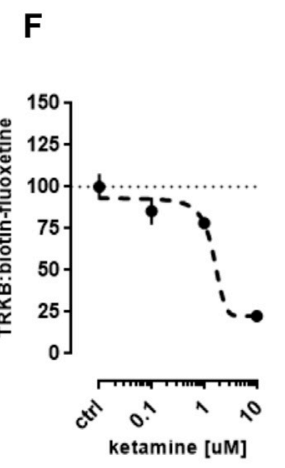

G

H

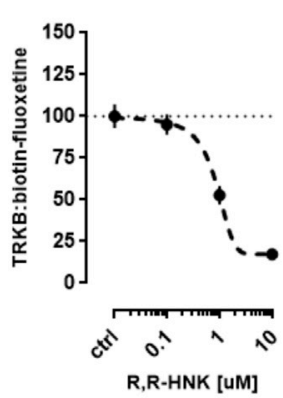

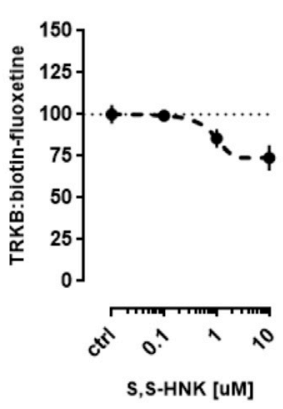

I

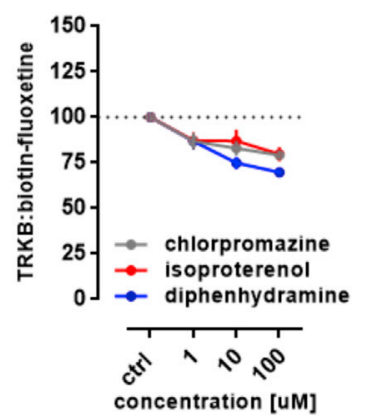

J

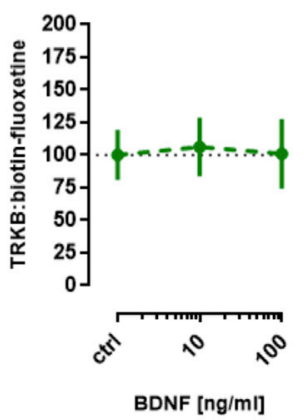

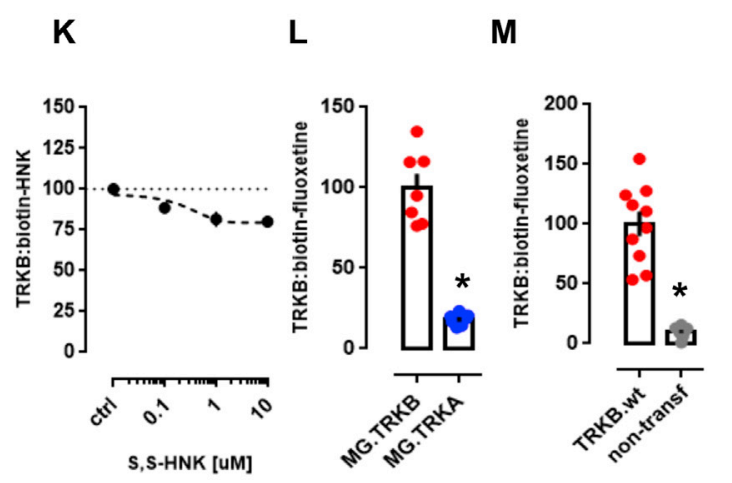

$\mathbf{N}$

0

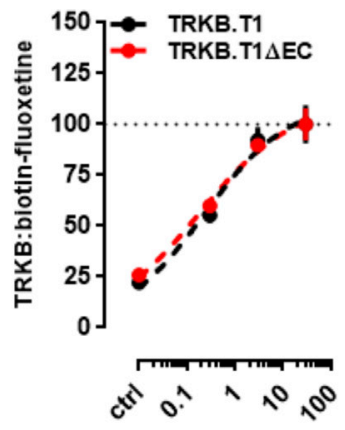

b-fluoxetine

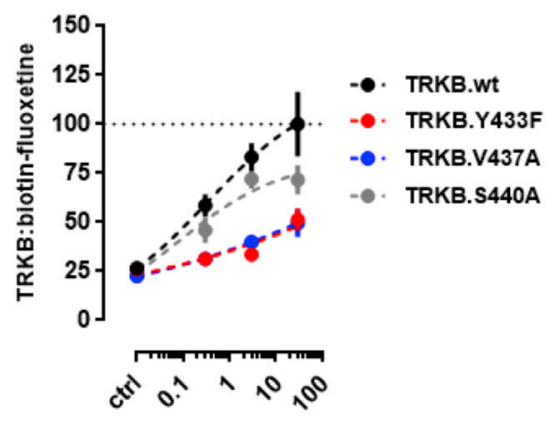

b-fluoxetine

Figure S4. Antidepressants bind to TRKB transmembrane domain, related to Figure 2

Lysate from HEK293T cells expressing TRKB were submitted to ligand binding assays. (A) Schematic representation of the biotinylated fluoxetine interaction with immobilized TRKB. (B-J) Biotinylated fluoxetine $(1 \mu M)$ interaction with TRKB is reduced by non-biotinylated (B) fluoxetine $(n=6 / g r o u p),(C)$ imipramine $(n=8 /$ group), (D) moclobemide ( $n=10 /$ group), (E) venlafaxine $(n=6 /$ group), (F) ketamine $(n=8 /$ group), (G) R,R-HNK ( $n=8 /$ group), but not reduced by (H) S,S-HNK ( $n=8$ /group), (I) chlorpromazine ( $n=8 /$ group), isoproterenol ( $n=8 /$ group) or diphenhydramine $(n=8 /$ group), or (J) BDNF ( $n=6 /$ group). (K) Biotinylated R,R-HNK $(1 \mu \mathrm{M})$ interaction with TRKB is not reduced by S,S-HNK ( $n=12$ /group). (L,M) Biotinylated fluoxetine interaction with (L) TRKA ( $n=7 /$ group) from MG87 cells, or (M) lysates from non-transfected HEK cells ( $n=10 /$ group) are negligible compared to TRKB. The interaction of biotinylated fluoxetine is not altered in ( $N$ ) TRKB lacking most of the intra and extracellular domains (TRKB.T1 $\triangle E C, n=12$ /group), but it is reduced by $(\mathrm{O}) \mathrm{V} 437 \mathrm{~A}$ and Y433F mutations, and partially attenuated by S440A ( $n=6$ /group). 
A

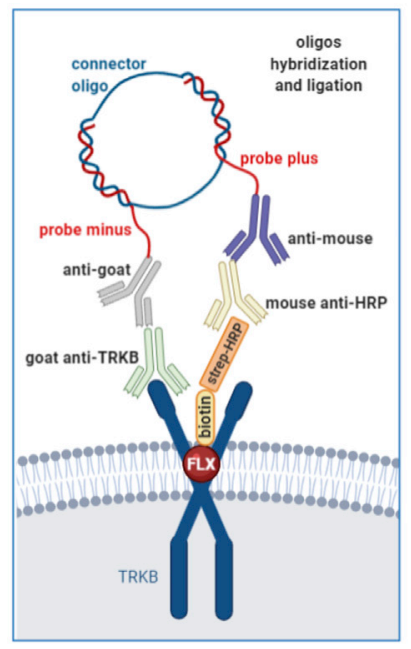

\section{EGFP}
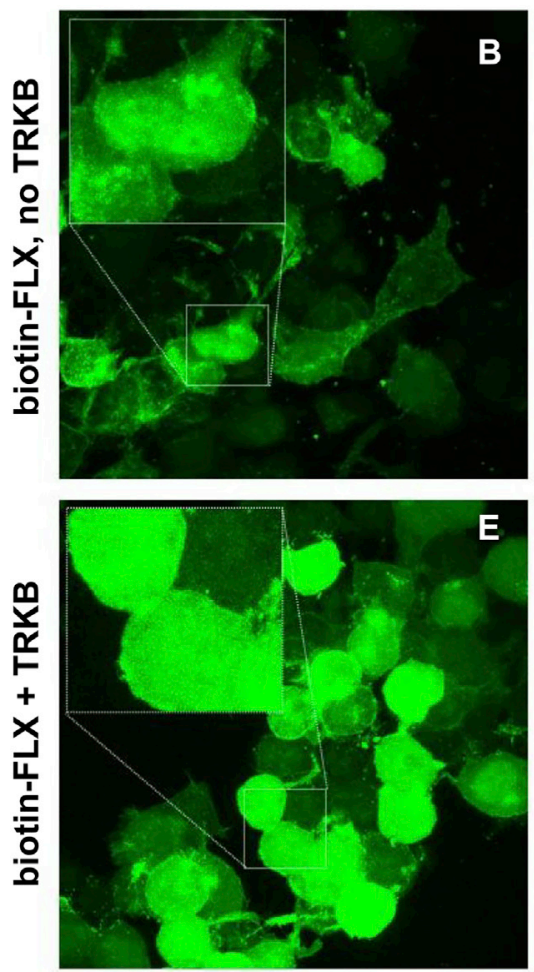

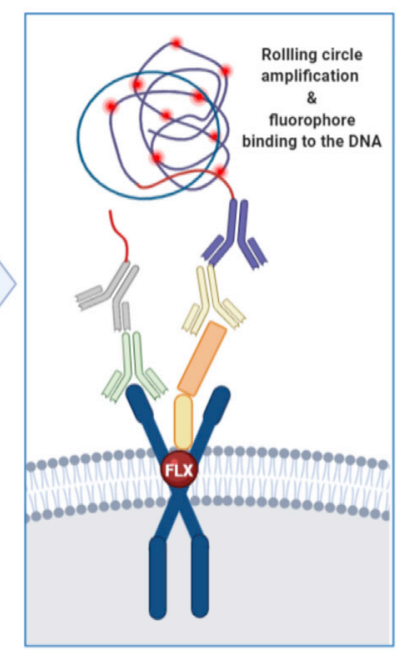

\section{DAPI}
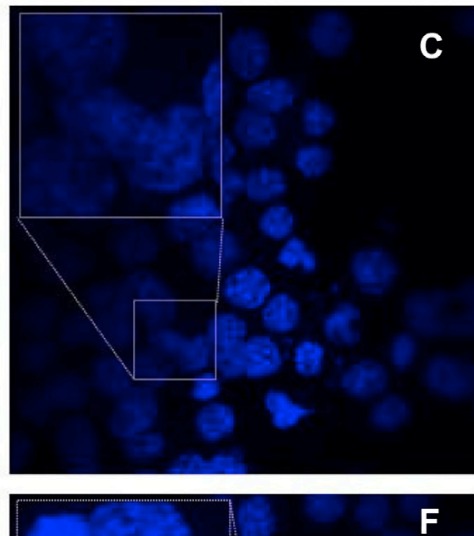

TRKB-FLX (PLA)
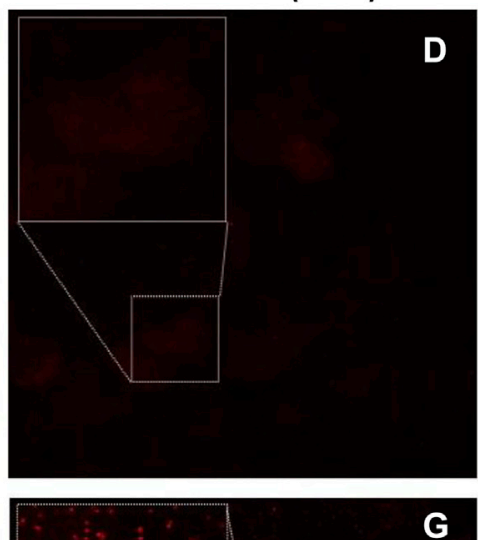

Figure S5. Antidepressants bind to TRKB in the intact cells, related to Figure 2

(A) Schematic representation of the in situ proximity ligation assay (PLA) between TRKB and biotinylated fluoxetine. (B-G) HEK cells were transfected to express TRKB and farnesylated GFP and were exposed to biotinylated fluoxetine (10uM/15min). The cells were fixed in PFA and the PLA reaction was conducted in permeabilized cells. (B-D) No signal from TRKB-FLX interaction was observed when cells were not transfected to express TRKB. (E-G) positive signal of TRKBFLX (PLA). Scale bar: $20 \mu \mathrm{m}$. Zoom in square: $2.5 \mathrm{x}$. 
A

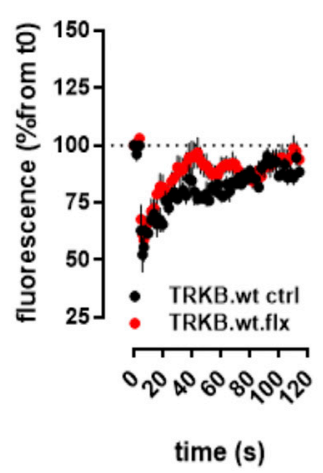

F

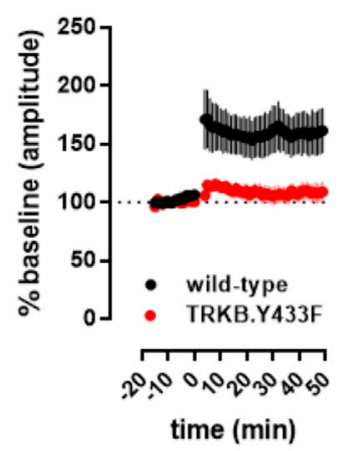

J

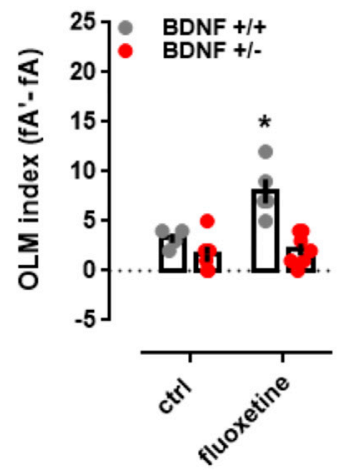

B

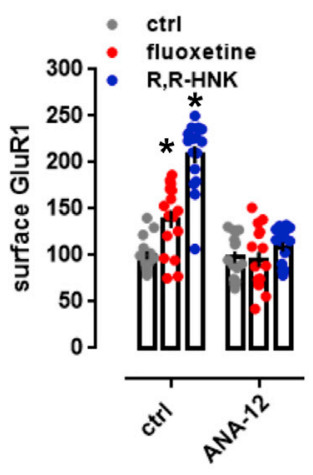

G

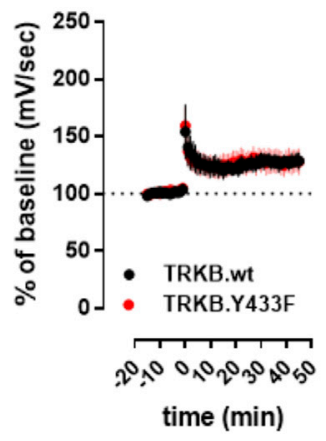

K

\section{HTT.ko}

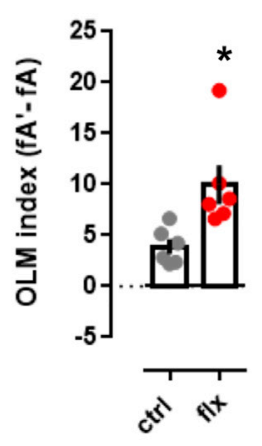

D
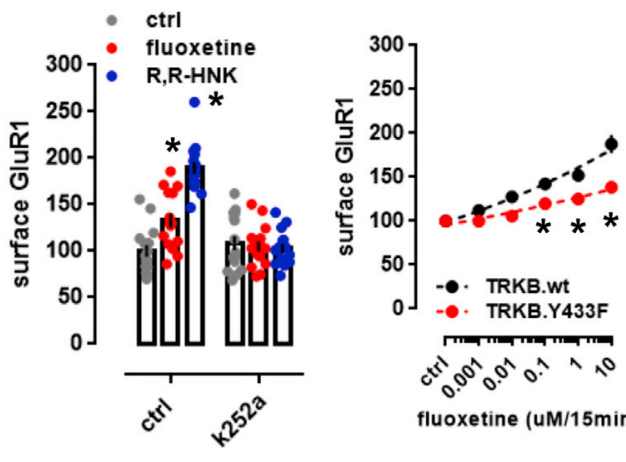

fluoxetine (uM/15min)
E

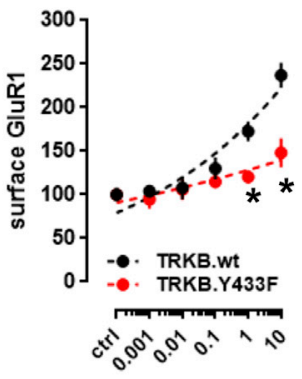

R,R-HNK (UM/15min)
H

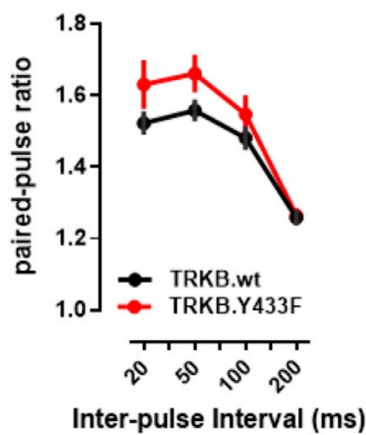

I

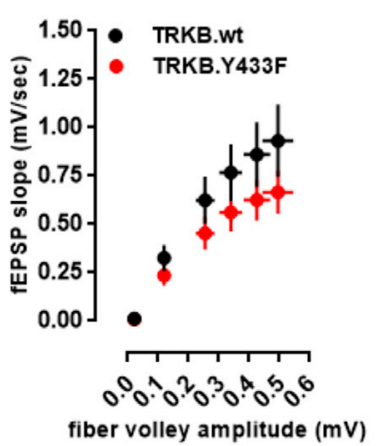

Figure S6. Antidepressants and cholesterol promote membrane trafficking and TRKB-mediated plasticity, related to Figures 4 and 5 (A) The fluorescence recovered after bleaching of GFP-TRKB in the neurite shaft of hippocampal neurons $[n=4-6$; interaction: $F(59,480)=0.7580, p=0.9061]$. (B-E) Fluoxetine- and R,R-HNK-induced increase in the surface levels of GluR1 subunit of AMPA receptors are prevented by $(B)$ ANA-12 [F(2,89) $=22.13$, $p<0.0001, n=15-16],(C) k 252 a[F(2,89)=27.83, p<0.0001, n=15-16]$ in rat cortical cells, and by $(D, E)$ the $Y 433 F$ mutation of TRKB $[f l u o x e t i n e: ~ F(5,132)=3.941$, $p=0.0023, n=12 /$ group; R,R-HNK: $F(5,132)=5.022, p=0.0003, n=12 /$ group] in mouse cortical cells. Data expressed as mean $\pm S E M$ of percentage from ctrl group. ${ }^{*} p<0.05$ from ctrl/TRKB.wt at the same dose. (F-l) Electrophysiological parameters of TRKB.Y433F mice. (F) TRKB.Y433F mutant mice display reduced theta-burst stimulus-induced changes in LTP [ $n=6$ /group; interaction: $F(61,610)=5.466 ; p<0.0001]$ but no changes in the (G) tetanic-stimulus-induced LTP [ $n=5$ /group; interaction: $F(60,480)=0.1333, p>0.9999]$, although a significant genotype effect was observed in $(H)$ paired-pulse facilitation [ $n=9 / g r o u p ;$ genotype: $F(1,64)=5.664, p=0.0203$; interaction: $F(3,64)=0.6356, p=0.5948]$ and $(I)$ input-output ratio [n = 9/group; genotype: $F(1,96)=6.388, p=0.0131$; interaction: $F(5,96)=0.3945, p=0.8515]$ no interaction was identified. Data expressed as mean \pm SEM of percentage from t0, baseline, or ctrl group. (J-K) Fluoxetine-induced $(15 \mathrm{mg} / \mathrm{kg} / 7$ days in drinking water) increased performance in OLM was prevented in mice $(\mathrm{J})$ heterozygous to BDNF [ $n=4-7$; interaction: $F(1,18)=6.878, p=0.0173]$, but not in $(K)$ animals lacking the serotonin transporter $[n=6 /$ group; $t(10)=2.962, p=0.0142]$. ${ }^{*} p<0.05$ from ctrl. 